\title{
A genetic system to study \\ the nuclear pore complex permeability barrier of the yeast Saccharomyces cerevisiae
}

\author{
Dissertation \\ for the award of the degree \\ "Doctor rerum naturalium" (Dr. rer. nat.) \\ of the Georg August Universität Göttingen
}

submitted by

Michael Ridders

born in

Krefeld, Germany

Göttingen 2012 
Members of the Thesis Committee:

Prof. Dr. Dirk Görlich, Reviewer

Department of Cellular Logistics

Max Planck Institute for Biophysical Chemistry

Prof. Dr. Gerhard Braus, Reviewer

Department of Molecular Microbiology \& Genetics

Georg August University Göttingen

Dr. Hans Dieter Schmitt

Research Group Membrane Transport in Yeast

Max Planck Institute for Biophysical Chemistry

Additional Members of the Extended Thesis Committee:

Prof. Dr. Detlef Doenecke

Department of Biochemistry I

Georg August University Göttingen

Prof. Dr. Volker Lipka

Department of Plant Cell Biology

Georg August University Göttingen

Prof. Dr. Michael Thumm

Department of Biochemistry II

Georg August University Göttingen

Date of Thesis submission: 30. April 2012

Date of the oral examination: 07. June 2012 


\section{Affidavit}

I hereby declare that this dissertation "A genetic system to study the nuclear pore complex permeability barrier of the yeast Saccharomyces cerevisiae" has been written independently with no other aids or sources than quoted.

This dissertation has not been submitted elsewhere for any academic award or qualification.

Michael Ridders

April, 2012

Göttingen, Germany 
However beautiful the strategy, you should occasionally look at the results.

WiNSTON ChURCHILL 


\section{Contents}

Contents $\quad$ v

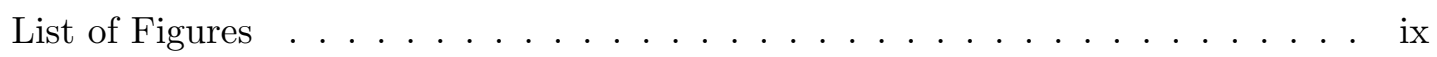

List of Tables ...................... . . .

Acknowledgements $\quad$ xi

Abstract $\quad$ xiii

1 Introduction 1

1.1 Composition of the Saccharomyces cerevisiae nuclear pore complex . . . . . 2

1.1.1 Integral Membrane Nucleoporins . . . . . . . . . . . . . . . . 5 5

1.1.2 Scaffold Nucleoporins _ . . . . . . . . . . . . . . . . 5

1.1.2.1 The Nup170 complex . . . . . . . . . . . . . . 5

1.1.2.2 The Nup84 complex . . . . . . . . . . . . . 6

1.1.2.3 The Nic96 complex _. . . . . . . . . . . . 6

1.1.2.4 The nuclear basket forming complex . . . . . . . . . 6

1.1.3 Barrier-forming Nucleoporins . . . . . . . . . . . . . . . 7

1.1.3.1 The Nsp1 complex . . . . . . . . . . . . . . . 9

1.1.3.2 Yeast homologs of vertebrate Nup98 . . . . . . . . . . 9

1.1.3.3 The Nup82 complex . . . . . . . . . . . . . . . . . . . . 11

1.1.3.4 Peripheral FG Nups . . . . . . . . . . . . . . . . . . 11

1.2 Models of nucleocytoplasmic transport across the NPC permeability barrier 13

1.2.1 The reduction of dimensionality model . . . . . . . . . . . . . . 13

1.2.2 The virtual gating model . . . . . . . . . . . . . . . . . . 13

1.2.2.1 The reverse collapse model . . . . . . . . . . . . . . 14

1.2.3 The selective phase model . . . . . . . . . . . . . . . . . . . . . . 14

1.2.4 The forest model . . . . . . . . . . . . . . . . . . . . . 15

1.3 Yeast genetics to study the NPC permeability barrier . . . . . . . . . 15 
1.4 Aim of this study . . . . . . . . . . . . . . . . . 18

\section{Results}

2.1 The combined deletions of FG domains from Nsp1p, Nup49p, and Nup57p are not lethal . . . . . . . . . . . . . . . . . . . . 21

2.1.1 Remaining scar domains result in a deleterious effect . . . . . . . . . 21

2.2 S. cerevisiae tolerates more FG domain deletions than expected . . . . . . . 23

2.3 Multi $\Delta \mathrm{FG}$ mutant strains can be used to assay in vivo functionality of $\mathrm{FG}$ domains at a given NPC anchor point . . . . . . . . . . . . . 24

2.3.1 FG domains at Nup98 homologous anchor position are essential . . . 25

2.3.1.1 The GLEBS domain can be functionally transplanted to Nsp1p . . . . . . . . . . . . . . . . . 25

2.3.1.2 Only Nup116p FG domains can functionally replace the Nup100p FG domain . . . . . . . . . . . . . . . . . 29

2.3.1.3 Only Nup100p FG domains can functionally replace the Nup116p FG domain . . . . . . . . . . . . . . . . . 31

2.3.2 Endogenous FG domains can functionally replace the Nsp1p FG

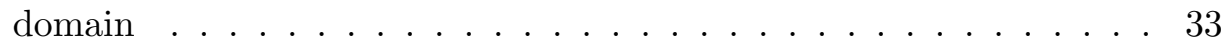

2.4 The Nup100p FG domain causes a dominant negative effect at various an-

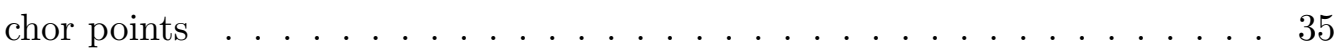

2.4.1 Nup100p FG domain fusion constructs are incorporated into the NPC 35

2.4.2 Nup100p FG domain reduces cell growth at various anchor domains 38

2.4.3 The negative effect is not caused by exceeding FG mass . . . . . . 38

2.4.4 The negative effect is not caused by an inappropriate connection to the anchor domain . . . . . . . . . . . . . . . . 40

2.5 FG domain mass is insufficient to predict viability . . . . . . . . . . . 40

\section{Discussion}

3.1 Experimental systems for the analysis of the permeability barrier . . . . . . 44

3.1.1 Differences among FG domains . . . . . . . . . . . . . . . . . . . 44

3.1.1.1 Limits of current experimental systems . . . . . . . . 45

3.1.2 New experimental systems to study the NPC permeability barrier . 47

3.1.3 Establishment of the yeast genetic system . . . . . . . . . . . . . 48

3.1.3.1 A new FG domain deletion strategy was needed . . . . . . 48

3.1.3.2 FG domains operate in a redundant manner . . . . . . . . 49 
3.1.3.3 FG domains of the Nsp1 subcomplex are not essential . . . 50

3.1.3.4 Re-evaluation of former results is needed . . . . . . . . 51

3.2 Role of FG domain anchor points . . . . . . . . . . . . . . . . . . 52

3.3 Role of FG domain cohesion for in vivo permeability barrier functionality . 53

3.3.1 Cohesive FG domains are essential for in vivo permeability barrier function . . . . . . . . . . . . . . . 53

3.3.2 Can the NPC channel be blocked by an excessive presence of cohesive FG mass? . . . . . . . . . . . . . . . . . . . . 55

3.4 Role of FG domain heterogeneity . . . . . . . . . . . . . . . . 55

3.4.1 Do less cohesive FG domains regulate the tightness of the yeast permeability barrier? . . . . . . . . . . . . 56

3.4.2 Vertebrates regulate the tightness of their permeability barrier . . . 56

3.5 Implications for proposed permeability barrier models . . . . . . . . . . 57

3.6 Perspectives . . . . . . . . . . . . . . . . . . . . . . . 59

4 Materials and Methods $\quad 61$

4.1 Materials . . . . . . . . . . . . . . . . . . . . . 61

4.1 .1 Chemicals . . . . . . . . . . . . . . . . 61

$4.1 .2 \quad$ Software . . . . . . . . . . . . . . . . . . 61

4.1 .3 Instruments . . . . . . . . . . . . . . . . . . . . 62

4.1 .4 buffers \& solutions . . . . . . . . . . . . . . . . . . . 62

4.1.5 Escherichia coli Media . . . . . . . . . . . . . . . 63

4.1.6 Escherichia coli Strains . . . . . . . . . . . . . . . . 63

4.1.7 Saccharomyces cerevisiae Media . . . . . . . . . . . . . 64

4.1.8 Saccharomyces cerevisiae Strains . . . . . . . . . . . . . . 65

4.1 .9 Oligonucleotides . . . . . . . . . . . . . . . . . 69

4.1 .10 Plasmids . . . . . . . . . . . . . . . . . . . . 69

4.2 Methods . . . . . . . . . . . . . . . . . . . 73

4.2.1 Standard Methods in Molecular Biology . . . . . . . . . . . . . . 73

4.2.1.1 Polymerase Chain Reaction (PCR) . . . . . . . . . . . 74

4.2.1.2 Mutagenesis Polymerase Chain Reaction . . . . . . . . 75

4.2.1.3 DNA Gel Electrophoresis . . . . . . . . . . . . . 75

4.2.1.4 Purification of DNA fragments from Agarose Gel and Concentration Determination . . . . . . . . . . . 76 
4.2.1.5 Enzymatic Restriction Digestion \& Ligation of DNA . . . . 76

4.2.1.6 Transformation of Escherichia coli with DNA . . . . . 76

4.2.1.7 Colony PCR with Escherichia coli cells . . . . . . . . . . . 77

4.2.1.8 Plasmid DNA Purification from Escherichia coli . . . . . . 77

4.2.1.9 DNA Sequencing . . . . . . . . . . . . . 77

4.2.2 Methods in Saccharomyces cerevisiae Genetics . . . . . . . . . . 77

4.2.2.1 General Culture and Storage of Saccharomyces cerevisiae . 78

4.2.2.2 Transformation of Saccharomyces cerevisiae with DNA . . 78

4.2.2.3 Genomic Gene Deletion . . . . . . . . . . . . . . . . 78

4.2.2.4 Genomic Deletion of FG Domains . . . . . . . . . . . 80

4.2.2.5 Preparation of Genomic DNA from Saccharomyces cerevisiae 81

4.2.2.6 Preparation of Plasmid DNA from Saccharomyces cerevisiae 81

4.2.2.7 Colony PCR with Saccharomyces cerevisiae cells . . . . . 83

4.2.2.8 Sporulation \& Tetrad Dissection . . . . . . . . . . . . . 83

4.2.2.9 FOA Complementation Assay . . . . . . . . . . . . . . 84

4.2.2.10 Temperature Sensitivity Assay . . . . . . . . . . . 85

4.2 .3 Confocal Microscopy . . . . . . . . . . . . . . . . . . . . 85 


\section{List of Figures}

1.1 Scheme of the nuclear pore complex model . . . . . . . . . . . . . . 3

1.2 Schematic representation of $S$. cerevisiae FG nucleoporins . . . . . . . . 8

$1.3 \quad$ FG domain deletion with Cre recombinase . . . . . . . . . . . . . 17

2.1 Effect of a deletion scar f . . . . . . . . . . . . . . . . . 22

2.2 Temperature sensitivity assay . . . . . . . . . . . . . . . . 24

2.3 Nsp1-GLEBSp fusion does not interfere with Nsp1p function . . . . . . 26

2.4 Nup116p becomes dispensable after GLEBS transplantation . . . . . . . . 28

$2.5 \quad$ Effect of Nup100p and Nup116p deletion . . . . . . . . . . . . . . . . 29

2.6 Subdivision of Nup100p FG domain . . . . . . . . . . . . . . . . . 30

2.7 Endogenous FG domains at the Nup100p anchor point . . . . . . . . . . 31

2.8 Endogenous FG domains at the Nup116p anchor point . . . . . . . . . . 32

2.9 Effect of a quadruplicated Nup145N FG domain . . . . . . . . . . . . . 33

2.10 YMR113 proof of principle . . . . . . . . . . . . . . . . 34

2.11 Endogenous FG domains at the Nsp1p anchor point . . . . . . . . . . . 34

2.12 Nup100p FG domain fusion proteins incorporate into the NPC . . . . . . 37

2.13 Nup100 FG domain fusion proteins show a dominant negative effect . . . 39

2.14 Increasing mass at the $\mathrm{Nsp1p}$ anchor point . . . . . . . . . . . . . . . . 40

$4.1 \quad$ Principle of gene replacement . . . . . . . . . . . . . . . . . . . 79

4.2 Principle of gene domain deletion via loop-in loop-out . . . . . . . . . . 82 


\section{List of Tables}

1.1 Overview of yeast \& vertebrate nups . . . . . . . . . . . . 4

2.1 Remaining FG mass in multi $\Delta$ FG mutant strains $\ldots \ldots \ldots$. . . . . . 41

$4.1 \quad$ S. cerevisiae strains used in this study . . . . . . . . . . . . 65

4.2 Plasmids used in FOA Assays . . . . . . . . . . . . . . . . 70

4.3 Additional plasmids used in this study . . . . . . . . . . . . . . . . 72

4.4 Gene deletion cassettes . . . . . . . . . . . . . . . . . . . . . 79

4.5 Constructs for $\Delta \mathrm{FG}$ domain insertion $\ldots \ldots \ldots \ldots \ldots$ 


\section{Acknowledgements - Danksagung}

Ich möchte all den Menschen danken, die mir bei der Ausfertigung dieser Arbeit geholfen und mich auf die ein oder andere Weise unterstützt haben.

So gilt mein Dank zunächst Prof. Dr. Dirk Görlich, der mich in seiner Gruppe willkommen hieß. In den Jahren unserer Zusammenarbeit hat er mir immer wieder das große Gesamtbild vor Augen gehalten, wenn ich mich auf Details fixierte und hat nie das Vertrauen in mein Projekt verloren. Mit seiner wissenschaftlichen Brillanz und seinem methodischen Sachverstand ist er ein beispielhaftes Vorbild.

Auch bedanke ich mich bei Prof. Dr. Gerhard Braus und Dr. Hans Dieter Schmitt, die als Mitglieder meines "thesis committee" durch ihr Engagement ihren Anteil am Erfolg dieser Arbeit geleistet haben.

Diese Arbeit wäre nicht möglich gewesen ohne die Unterstützung von Steffen Frey. Nicht genug, dass ich auf seine umfangreiche Konstrukt-Sammlung zugreifen durfte, er hat sich auch mit zahllosen Ideen und Anregungen stets für den Erfolg des Projekts eingesetzt und stand mir bei allen Fragen mit offener und stets konstruktiver Kritik zur Seite.

Ich möchte mich besonders herzlich bei "meinen Mädels" von Lab V bedanken. Heike, Cathrin, Susanne und Jenny, sowie seit Kurzem auch Tino, teilten in den Jahren Leid \& Freud, Lust \& Frust sowie Puffer \& Medien mit mir. Obwohl ich ihnen durch mein Gemurmel, meinen speziellen Charme und meinen Humor bestimmt oft viel abverlangte, nahmen sie es immer leicht auf. Mein Dank geht an Euch nicht zuletzt für die immer freundschaftliche, heitere und vertrauensvolle Atmosphäre im Labor, die angeregten Diskussionen über wissenschaftliche, ernste und nicht ganz so ernste Themen sowie für die Zeit, die wir eben nicht im Labor verbrachten. 
Darüber hinaus haben mich Susanne und Heike bei zahlreichen Klonierungen unterstützt. Besonders zu erwähnen ist auch Gabi, die für mich unzählige Midis präpariert hat und immer noch begeistert nach mehr verlangt, sowie Uwe und Elisabeth, die für uns alle stets fleißig autoklavierten, spülten, alliquotierten und sowieso Ordnung hielten. Ein Danke auch an Connie, die den Laden hier am Laufen hält.

Ohnehin möchte ich mich bei der gesamten Abteilung Görlich für die einmalige Arbeitsatmosphäre, die Diskussionen sowie die Anregungen und Ideen bedanken.

Darüber hinaus möchte meine Dankbarkeit gegenüber GGNB für seine finanzielle und administrative Unterstützung ausdrücken. Das Engagement der Beteiligten um den Aufbau einer großartigen Graduiertenschule kann man gar nicht genug hervorheben.

Ebenso bedanke ich mich bei allen, die meine Zeit in Göttingen zu mehr als zu reiner Arbeitszeit werden ließ.

Ganz besonders möchte ich meinen Eltern, Großeltern und Brüdern danken. Sie haben mir das mit auf den Weg gegeben, was man als Wissenschaftler unbedingt benötigt - innere Ruhe, Selbstvertrauen und die Kraft auch größte Frustrationen zu überstehen. Und auch wenn sie sich vielleicht nur schwer vorstellen können, was ich hier in Göttingen getan habe, so vermittelten sie mir doch immer das Gefühl, dass sie sich sicher sind, dass ich es schaffen werde.

Last but not least möchte ich mich von ganzem Herzen bei Katrin bedanken, die seit mehr als zwei Jahren an meiner Seite steht und mit ihrer Liebe mein Leben erhellt und bereichert.

\section{Danke Euch allen!}




\section{Abstract}

All nucleocytoplasmic transport occurs through nuclear pore complexes (NPCs). To hinder uncontrolled mixing of cytoplasmic and nuclear content, NPCs maintain the permeability barrier that suppresses passage of inert macromolecules but allows facilitated translocation of even very large cargoes provided these are bound to nuclear transport receptors (NTRs). About a third of the proteins that built up the NPC contains so-called FG domains that are essential for establishing the NPC permeability barrier. NTRs bind to FG motifs and this interaction is essential for their privileged passage. How this binding accelerates NPC passage is one of the enigmas in the field.

FG domains are quite diverse, differing in their cohesiveness, in their FG motifs, their length, their charge distribution, and in the sequence of the spacers between the individual FG motifs. The biophysical and biochemical properties of individual FG domains have been well studied in vitro. The significance of most of the observed features for the functionality of the permeability barrier in vivo, however, so far remained unclear. Previously, the in vivo significance of individual FG domains was studied by generating $S$. cerevisiae deletion strains lacking multiple FG domains in various combinations. We show that previously reported lethal phenotypes of the combined deletion of FG domains were not exclusively caused by the deletions but were a result of the previously applied deletion strategy. Moreover, with an alternative deletion strategy, we show that $S$. cerevisiae tolerates more FG domain deletions than expected so far.

The focus of my $\mathrm{PhD}$ work has been to elucidate which FG domain features are essential for permeability barrier function in vivo. We established an in vivo system allowing to analyze the functional contribution of individual FG domains by testing the consequences of mutations, deletions, or exchange of these domains for cell viability. Employing this in vivo system, we find that the very cohesive FG domains of Nup100p and Nup116p play a prominent role in maintaining the permeability barrier. We show that not all FG domains can functionally replace these prominent FG domains, suggesting that NTR binding alone is not sufficient to explain the functionality of the permeability barrier. Additionally, we show that the anchor points for FG domains within the NPC are not equivalent.

Our findings support models of permeability barrier functionality that rely on the cohesiveness of FG domains. Based on our results, we, however, assume that both the excessive presence of cohesive FG mass as well as the absence of very cohesive FG domains is incompatible with viability. 


\section{Introduction}

Eukaryotic life is characterized by the separation of cellular processes into distinct compartments. Presumably, this compartmentalization was a prerequisite for the evolution of multicellular organisms as it allows a higher degree of organization and regulation.

A highly specialized compartments is the nucleus that gives eukaryotes their name ${ }^{1}$. It is surrounded by the double membrane of the nuclear envelope (NE) that separates nuclear processes, like transcription, from cytoplasmic processes, e.g. translation.

This spatial separation necessitates tremendous bidirectional transport processes, since on the one hand the proteins of the transcription machinery are synthesized in the cytoplasm whereas on the other hand the information for protein synthesis, i.e. the mRNA templates, originate from the DNA stored in the nucleus. Additionally, ribosomal subunits are assembled in the nucleus and need to cross the NE before they can fulfill their function in the cytoplasm.

These transport processes occur via nuclear pore complexes (NPCs) that perforate the nuclear envelope (Bahr and Beermann, 1954) and therewith provide the transport route between cytoplasm and nucleoplasm (Feldherr, 1962).

To avoid uncontrolled intermixing of cytoplasm and nucleoplasm, NPCs establish the so-called "permeability barrier" (Paine et al., 1975). This barrier excludes inert macromolecules $>40 \mathrm{kDa}$ or $\geq 5 \mathrm{~nm}$ in diameter (Paine and Feldherr, 1972; Mohr et al., 2009), but at the same time permits facilitated passage of even very large cargoes, provided these are bound to nuclear transport receptors (reviewed by Görlich and Kutay, 1999; Fried and Kutay, 2003).

NPCs are large structures of $\sim 66 \mathrm{MDa}$ in the yeast S. cerevisiae (Rout and Blobel, 1993) and of $\sim 125 \mathrm{MDa}$ in higher eukaryotes (Reichelt et al., 1990) that exhibit an eight-fold rotational symmetry (Wischnitzer, 1958; Gall, 1967). They are build up by multiple copies

${ }^{1}$ from the Greek $\epsilon v$ (eu, "good") and $\kappa \alpha \rho u \omega \tau o \varsigma$ (karyotos, "nut-like") 
of $\sim 30$ different proteins (Rout et al., 2000; Cronshaw et al., 2002), generally referred to as nucleoporins (Nups).

Electron microscopy studies visualized NPCs from Xenopus laevis (Hinshaw et al., 1992; Akey and Radermacher, 1993; Stoffler et al., 2003), Saccharomyces cerevisiae (Yang et al., 1998; Kiseleva et al., 2004), Chironomus thummi (Kiseleva et al., 1998), and Dictyostelium discoideum (Beck et al., 2004, 2007) and thereby showed that the overall structure of the NPC is evolutionary conserved. The central core of the NPC is formed by two nearly identical rings that are embedded in the NE. On the cytoplasmic side, filaments emanate from the NPC into the cytoplasm, whereas at the nucleoplasmic side filaments build up a structure called the nuclear basket (see figure 1.1 A for a consensus model).

Interestingly, the atomic structures from several Nups obtained in the recent past years (see e.g. Brohawn et al., 2008; Nagy et al., 2009; Whittle and Schwartz, 2009) show a striking similarity to the structural organization of membrane vesical-coating proteins (reviewed by Field and Dacks, 2009; Onischenko and Weis, 2011; Aitchison and Rout, 2012). These observations support the previously stated "protocoatomer hypothesis", which proposes a common ancestor of NPCs and coated vesicles (Devos et al., 2004).

\subsection{Composition of the Saccharomyces cerevisiae nuclear pore complex}

In general, Nups can be divided into three groups: (i) Nups that are integrated into the membrane of the NE, (ii) structural Nups that form the NPC scaffold and (iii) FG Nups that enable proper NPC function by forming the NPC permeability barrier.

Most of the Nups are organized into distinct subcomplexes that are thought to be the building blocks of the NPC (for a schematic overview see figure 1.1 B).

Many Nups were originally discovered and studied in the yeast Saccharomyces cerevisiae (reviewed by Fabre and Hurt, 1997). In the following, the term "yeast" refers to the yeast S. cerevisiae if not otherwise stated.

For comparison, table 1.1 provides an overview of Nups from yeast and vertebrates. 


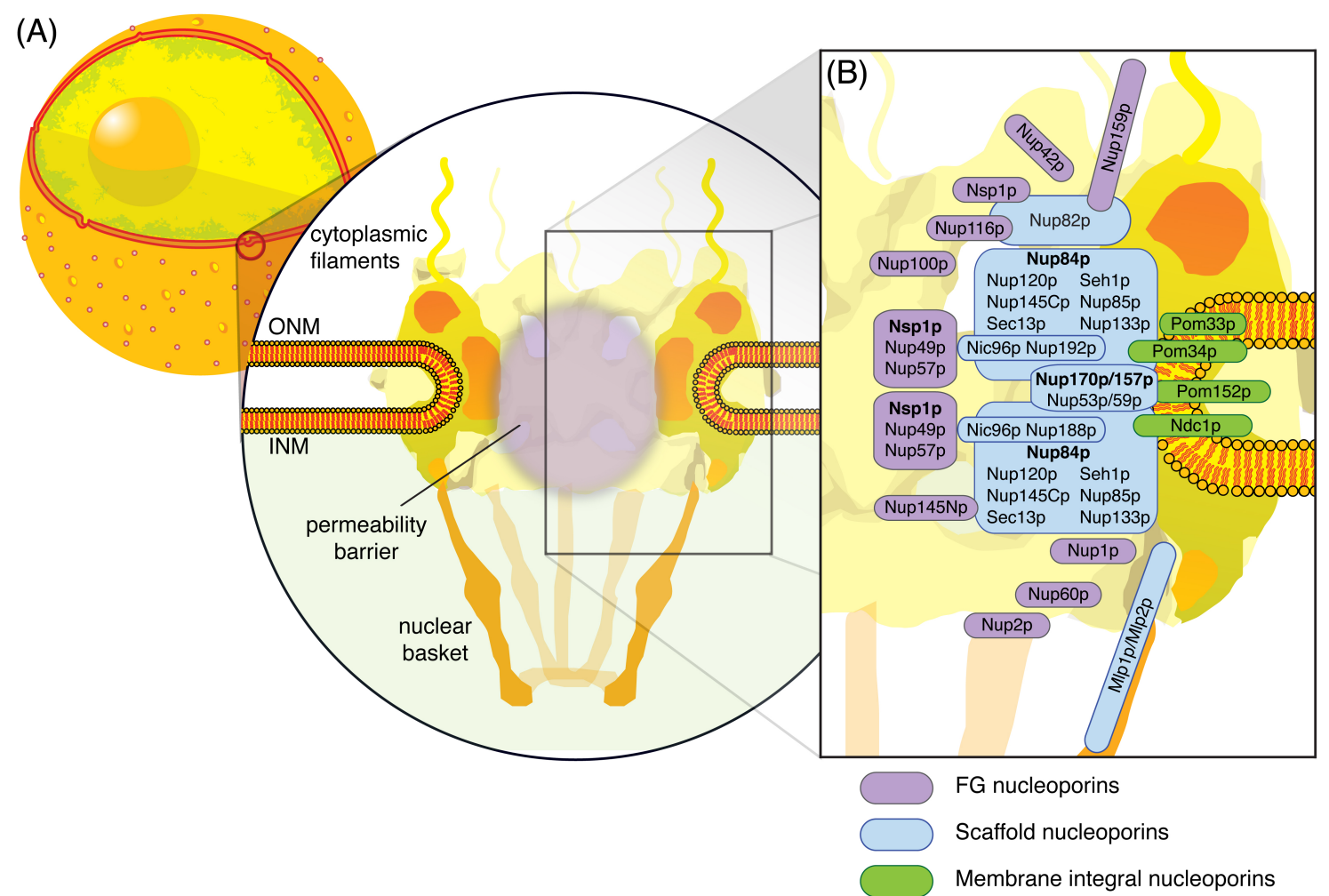

Figure 1.1: Schematic representation of the nuclear pore complex and individual Nups

within the NPC. (A) The NPC is embedded in the nuclear envelope. Its main structural components include the core scaffold, the permeability barrier, the cytoplasmic filaments, and the nuclear basket. (ONM - Outer Nuclear Membrane, INM - Inner Nuclear Membrane)

(B) Nucleoporins can be sorted into three different groups: integral membrane nucleoporins (green), rigid scaffold nucleoporins (blue), and FG motif-containing nucleoporins (purple). The grouping of individual nucleoporins indicates their organization in biochemically characterized subcomplexes. Please note that the representation does not reflect the actual stoichiometric ratio nor the size of individual proteins or structures.

(The figure is based on an artwork created for Wikipedia by Mike Jones. This image is licensed under the Creative Commons Attribution-Share Alike 2.5 Generic license.) 
Table 1.1: S. cerevisiae nups and their homologs in vertebrates. Summary of nups that constitute the NPC. Homology of yeast and vertebrate nups is listed according to Hetzer et al. (2005); D'Angelo and Hetzer (2008); Brohawn et al. (2009). "-" indicates that no homolog has been described.

\begin{tabular}{|c|c|c|c|}
\hline S. cerevisiae & Vertebrates & Nucleoporin type & Subcomplex in yeast \\
\hline Nup84p & Nup107 & Scaffold nucleoporin & \\
\hline Nup85p & Nup85/Nup75 & Scaffold nucleoporin & \\
\hline Nup120p & Nup160 & Scaffold nucleoporin & \\
\hline Nup133p & Nup133 & Scaffold nucleoporin & Nup84 complex \\
\hline Nup145Cp & Nup96 & Scaffold nucleoporin & \\
\hline Sec13p & Sec13 & Scaffold nucleoporin & \\
\hline Seh1p & Seh1 & Scaffold nucleoporin & \\
\hline Nic96p & Nup93 & Scaffold nucleoporin & \\
\hline Nup188p & Nup188 & Scaffold nucleoporin & Nic96 complex \\
\hline Nup192p & Nup205 & Scaffold nucleoporin & \\
\hline Nup53p & Nup35 & Scaffold nucleoporin & \\
\hline Nup59p & Nup35 & Scaffold nucleoporin & Nup170 complex \\
\hline Nup157p & Nup155 & Scaffold nucleoporin & \\
\hline Nup170p & Nup155 & Scaffold nucleoporin & \\
\hline Nup49p & Nup58 \& Nup45 & FG nucleoporin & Nsp1 complex \\
\hline 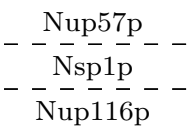 & $\begin{array}{l}--\frac{\operatorname{Nup} 54}{\operatorname{Nup} 62---} \\
--\frac{-}{\operatorname{Nup} 98}---\end{array}$ & 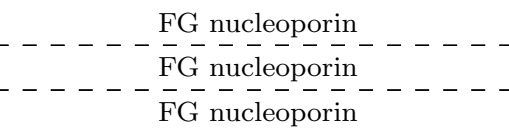 & Nsp1 \& Nup82 complex \\
\hline Nup82p & Nup88 & Scaffold nucleoporin & Nup82 complex \\
\hline Nup159p & Nup214/CAN & FG nucleoporin & \\
\hline Mlp1p & Tpr & Scaffold nucleoporin & \\
\hline Mlp2p & Tpr & Scaffold nucleoporin & Nuclear basket \\
\hline Nup1p & Nup153 & FG nucleoporin & forming complex \\
\hline Nup2p & Nup50 & FG nucleoporin & \\
\hline Nup60p & - & FG nucleoporin & \\
\hline Nup42p & NLP1/CG1 & FG nucleoporin & \\
\hline Nup100p & Nup98 & FG nucleoporin & \\
\hline Nup145Np & Nup98 & FG nucleoporin & \\
\hline Ndc1p & Ndc1 & Integral membrane nucleoporin & \\
\hline Pom33p & - & Integral membrane nucleoporin & \\
\hline Pom34p & - & Integral membrane nucleoporin & \\
\hline Pom152p & - & Integral membrane nucleoporin & \\
\hline- & gp210 & Integral membrane nucleoporin & \\
\hline- & Pom121 & Integral membrane \& FG nucleoporin & \\
\hline- & Aladin & Scaffold nucleoporin & \\
\hline- & Nup37 & Scaffold nucleoporin & \\
\hline- & Nup43 & Scaffold nucleoporin & \\
\hline- & Ganp & FG nucleoporin & \\
\hline- & Nup358/RanBP2 & FG nucleoporin & \\
\hline
\end{tabular}




\subsubsection{Integral Membrane Nucleoporins}

Transmembrane Nups anchor the NPC into the NE. In yeast, these are Ndc1p (Thomas and Botstein, 1986), Pom152p (Wozniak et al., 1994), Pom33p (Chadrin et al., 2010), and Pom34p (Rout et al., 2000).

Ndc1p localizes to the NPC and to the spindle pole body (Chial et al., 1998) and it is essential for spindle pole body duplication (Winey et al., 1993). The depletion of Ndc1p in interphase results only in a partial mislocalization of NPC components (Madrid et al., 2006).

Pom33p was found to be dynamically associated with the NPC and a POM33 deletion impairs NPC distribution (Chadrin et al., 2010).

The deletion of genes of the other transmembrane Nups does not affect cell viability (Wozniak et al., 1994; Giaever et al., 2002). Those deletions are only synthetically lethal when combined with mutant alleles of a number of other Nups (Miao et al., 2006; Chadrin et al., 2010).

\subsubsection{Scaffold Nucleoporins}

The scaffold nucleoporins provide the structural framework of the NPC. They are interacting with both, the transmembrane Nups and the barrier forming Nups (see figure 1.1 B).

\subsubsection{The Nup170 complex}

Ndc1p, Pom34p, and Pom152p bind to Nup53p and Nup59 (both originally identified by Rout et al., 2000). Nup53p and Nup59 then interact with Nup157p and Nup170p (Kenna et al., 1996; Lusk et al., 2002; Onischenko et al., 2009).

NUP157 and NUP170 are homologous genes and only one or the other is required for growth (Aitchison et al., 1995b). Their deletion does not seem to affect existing NPCs but hinders formation of new pores. Thus, it was proposed that they are required for the assembly of newly forming NPCs (Makio et al., 2009).

It was suggested that Nup170p and Nup157p may be part of a morphologically symmetrical but functionally distinct subcomplex of the NPC (Aitchison et al., 1995b). 


\subsubsection{The Nup84 complex}

A well characterized NPC subcomplex is the heptameric Nup84 complex that is build up by Nup84p, Nup85p, Nup120p, Nup133p, Nup145Cp, Seh1p, and Sec13p (Siniossoglou et al., 1996, 2000; Lutzmann et al., 2002). Structural similarities between Nup84 complex members and proteins of vesicle coating complexes suggest that all of these complexes have a common function in curving membranes (Devos et al., 2004; Brohawn et al., 2008; Nagy et al., 2009).

The Nup84 complex exhibits a Y-shaped structure in negative staining transmission electron microscopy (Siniossoglou et al., 2000; Fernandez-Martinez et al., 2012) with Nup120p forming the one and a Seh1p-Nup85p heterodimer forming the other arm of the Y (Lutzmann et al., 2002).

Although the Nup84 complex is essential for NPC formation (Siniossoglou et al., 1996), individual genes encoding Nup84 complex members can be deleted resulting in mRNA export defects (Aitchison et al., 1995a; Goldstein et al., 1996; Siniossoglou et al., 1996).

\subsubsection{The Nic96 complex}

The third subcomplex of the NPC scaffold is the Nic96 subcomplex constituted of Nic96p, Nup188p, and Nup192p (Grandi et al., 1993; Nehrbass et al., 1996; Zabel et al., 1996; Kosova et al., 1999).

Nic96p was originally identified by its interaction with the Nsp1 subcomplex (Grandi et al., 1993, see section 1.1.3.1) and it is required for NPC formation (Gomez-Ospina et al., 2000). Both, Nup188p and Nup192p were identified by their functional interaction with the Cterminus of Nic96p (Zabel et al., 1996; Kosova et al., 1999). NUP192 is essential (Kosova et al., 1999), whereas NUP188 is not essential but shows synthetic lethality when its deletion is combined with a mutant allele of NIC96 (Zabel et al., 1996).

No direct interaction of Nup188p and Nup192p was found so far, thus it was proposed that both associate independently with distinct copies of Nic96p (Alber et al., 2007).

\subsubsection{The nuclear basket forming complex}

Besides the previously mentioned structural subcomplexes of the NPC core, other structural elements are represented by Mlp1p (Kölling et al., 1993) and Mlp2p (de Castillia et al., 1999).

Mlp1p and Mlp2p are thought to assemble the yeast nucleoplasmatic basket structure by 
forming filamentous structures that reach into the nucleoplasm (de Castillia et al., 1999; Feuerbach et al., 2002; Kiseleva et al., 2004). This basket structure is presumably anchored to the NPC core via Nup60p (Feuerbach et al., 2002).

$M L P 1$ and $M L P 2$ can be deleted simultaneously without affecting cell viability (de Castillia et al., 1999). They are homologs to the vertebrate gene coding the nuclear basket protein Tpr (Cordes et al., 1997; de Castillia et al., 1999).

\subsubsection{Barrier-forming Nucleoporins}

Approximately one third of all nucleoporins comprise domains with multiple repeats of phenylalanine $(\mathrm{F})$ glycine $(\mathrm{G})$ dipeptide motifs that are separated by hydrophilic spacer sequences. These domains are referred to as "FG repeat domains" or "FG domains". FG domains represent $\sim 12 \%$ of the NPC mass (Yamada et al., 2010) and can be sorted according to the exact amino acid composition of an FG motif, with "FG", "GLFG", "FxFG", or "FxF" (where " $\mathrm{x}$ " stands for any amino acid) being the most prominent ones in yeast (Rout and Wente, 1994; Strawn et al., 2004).

In total, eleven Nups with FG domains were identified in yeast (Rout et al., 2000, see figure 1.2).

FG domains are considered to adopt a non-globular structure (Denning et al., 2002, 2003). However, based on the amino acid composition of the FG domains, distinct categories of intrinsically disordered structures were proposed (Yamada et al., 2010).

In contrast to the scaffold Nups or the non-FG domains of barrier-forming Nups, the amino acid sequences surrounding FG motifs are poorly conserved among eukaryotes. It was therefore proposed that they evolved roughly five times faster compared to structured domains (Denning and Rexach, 2007).

FG domains provide the binding sites for nuclear transport receptors (NTRs) and binding of NTRs to FG motifs is essential for facilitated NPC passage (Iovine et al., 1995; Radu et al., 1995; Bayliss et al., 1999, 2000; Bednenko et al., 2003; Isgro and Schulten, 2005). Aside from binding NTRs, FG domains were shown to form intramolecular and intermolecular interactions (Frey et al., 2006; Patel et al., 2007; Frey and Görlich, 2009; Ader et al., 2010; Yamada et al., 2010). The interactions of Nsp1p FG domains are best characterized. Recombinantly expressed Nsp1p FG domain was found to form macroscopic hydrogels by hydrophobic inter-repeat interactions (Frey et al., 2006; Ader et al., 2010). Additionally, the asparagine-rich spacer regions of the N-terminal FG domain of Nsp1p are engaged in amyloid-like intermolecular $\beta$-sheets (Ader et al., 2010). 
Nup42p
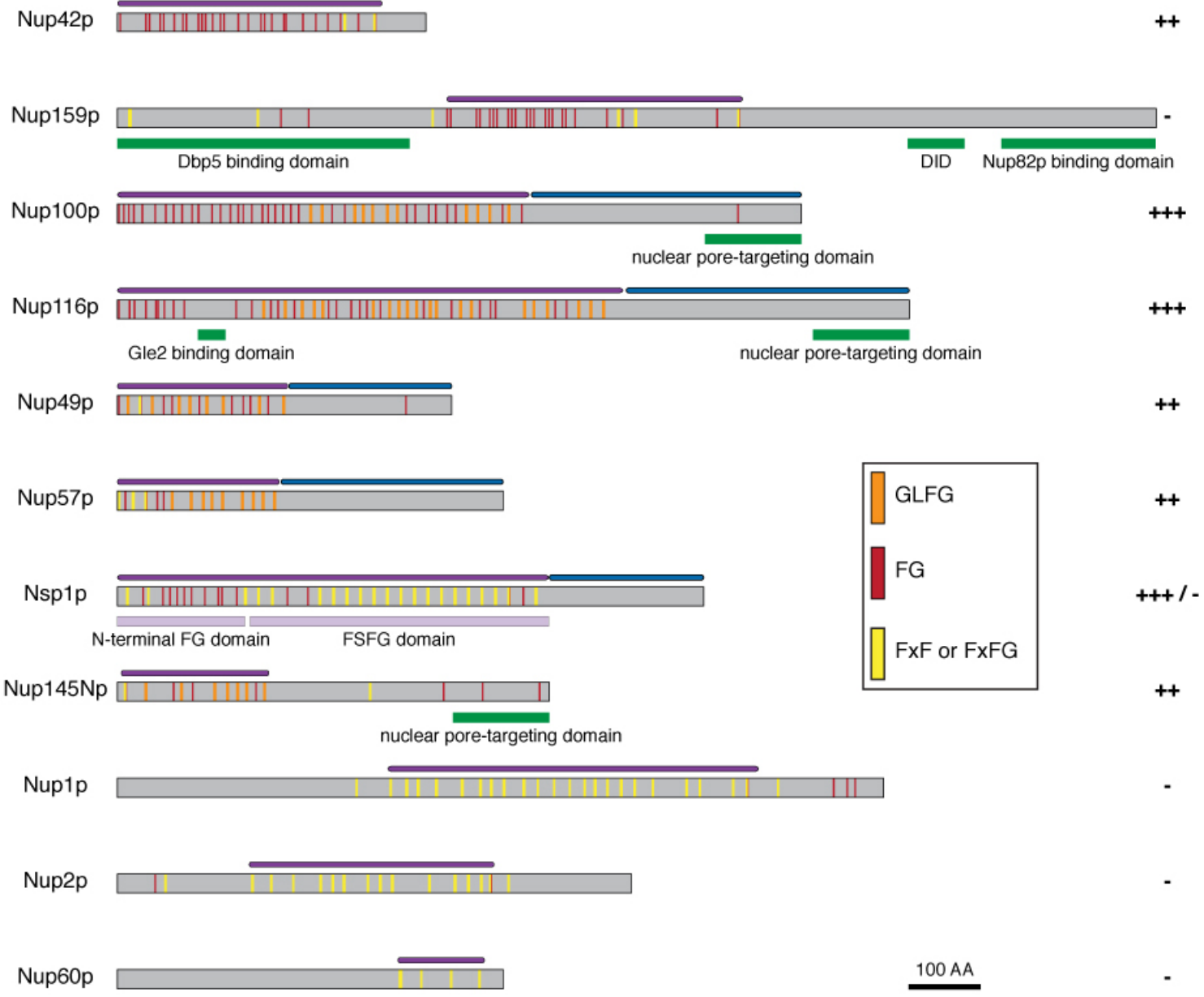

Figure 1.2: Schematic representation of $S$. cerevisiae FG nucleoporins: FG nucleoporins (grey boxes) were drawn to scale. Individual FG motifs are indicated as colored vertical strokes according to their exact sequence. FG domains (purple) and NPC anchor domains (blue) are indicated according to their definition in this study. Additional nucleoporin domains mentioned in the text are depicted as green boxes and the FG subdomains of Nsp1p are shown as lilac boxes. The cohesiveness of depicted FG domains was extrapolated from Frey et al. (2006); Patel et al. (2007); Frey and Görlich (2009); Ader et al. (2010); Yamada et al. (2010) and is indicated with (+++) very cohesive, (++) less cohesive, (+) slightly cohesive, and (-) non-cohesive. DID: dynein light chain interacting domain 
Anchored to the NPC scaffold by smaller structured domains, FG domains radiate into the central channel of the NPC (Grote et al., 1995) and are thought to form the permeability barrier that controls nucleocytoplasmic translocations (Ribbeck and Görlich, 2001; Frey and Görlich, 2007, 2009). It has been estimated that more than 1000 copies of FG repeats are within one NPC, and that their local concentration in the interior of the central channel approximates $50 \mathrm{mM}$ (Bayliss et al., 1999).

FG domains are essential for cell viability (Strawn et al., 2004). However, the deletion of individual FG Nup coding genes give rise to a variety of transport defects (reviewed e.g. in Fabre and Hurt, 1997).

\subsubsection{The Nsp1 complex}

The Nsp1 subcomplex was the first identified NPC subcomplex in yeast (Grandi et al., 1993, 1995). It is composed of the essential proteins Nsp1p (Hurt, 1988), Nup49p (Wimmer et al., 1992; Wente et al., 1992), and Nup57p (Grandi et al., 1995). Assembly of the complex occurs via the C-terminal coiled-coil domains of the complex members (Grandi et al., 1995; Schlaich et al., 1997; Bailer et al., 2001). These domains bind to the N-terminus of Nic96p and thereby attach the complex to the central part of the NPC scaffold (Grandi et al., 1995; Schrader et al., 2008, see figure 1.1 B). Notably, the NPC-binding site for Nsp1p on Nic96p is not required for cell viability, even though its deletion results in a severe growth defect (Schrader et al., 2008).

The FG domain of Nsp1p can be divided into two parts, namely (i) the highly cohesive Nterminal part with a low content of charged amino acids consisting mainly of "FG" motifs, and (ii) the more $\mathrm{C}$-terminal part of the FG domain with a higher content of charged amino acids and "FSFG" being the predominant motif (Ader et al., 2010; Yamada et al., 2010). The FG domains of Nup49p and Nup57p both belong to the "GLFG" family, being cohesive with a low content of charged amino acids (Wente et al., 1992; Yamada et al., 2010) (see figure 1.2). Nsp1p, but not Nup49p or Nup57p, is additionally part of the Nup82p complex (Belgareh et al., 1998, see below).

\subsubsection{Yeast homologs of vertebrate Nup98}

Nup145Np is generated in vivo by self-cleavage of the precursor protein Nup145p (Teixeira et al., 1997). The resulting C-terminal protein is Nup145Cp which is incorporated 
into the Nup84 subcomplex (Teixeira et al., 1997). The autocatalytic cleavage site within Nup145p is conserved throughout evolution (Teixeira et al., 1997) and allows the identification of homologous in virtually all eukaryotes (Broder Schmidt, personal communication and Neumann et al., 2010).

The vertebrate homolog of NUP145 is NUP98 (Powers et al., 1995; Radu et al., 1995). Like Nup145Np, Nup98 is generated by self-cleavage of a precursor protein (Fontoura et al., 1999). Moreover, S. cerevisiae possesses two additional homologs of NUP98, namely NUP100 and NUP116 (Fabre et al., 1994; Wente and Blobel, 1994; Powers et al., 1995). But Nup100p and Nup116p are not generated by self-cleavage of a precursor protein. NUP100 and NUP116 are both flanked by a histidine tRNA gene and a transposon element suggesting that they arose by gene duplication (Wente et al., 1992).

Nup100p, Nup116p, Nup145Np, and Nup98 share an N-terminally localized "GLFG" type FG domain (Wimmer et al., 1992; Wente et al., 1992; Wente and Blobel, 1994; Fabre et al., 1994) and the conserved autocatalytic domain, which functions as nuclear pore-targeting domain (Bailer et al., 1998; Ho et al., 2000; Hodel et al., 2002).

However, Nup100p, Nup116p and Nup145Np do not anchor into the NPC via the same proteins. Nup145Np was shown to bind to the Nup84 subcomplex through Nup145C (Ratner et al., 2007; Lutzmann et al., 2005) and a biochemical analysis implied that neither Nup100p nor Nup116p can attach to this binding partner in vivo (Ratner et al., 2007). Nup116p binds to the Nup82 subcomplex (Ho et al., 2000; Bailer et al., 2000). Additionally, a yet unknown binding site for Nup116p was proposed, since the deletion of its nuclear pore-targeting domain leads to a reduced but still observable localization at the nuclear rim, indicative for NPC incorporation (Yoshida et al., 2011). This assumption is well in line with the observation that a simultaneous deletion of all three nuclear pore-targeting domains results only in a severe temperature sensitive phenotype (Fabre et al., 1994). The binding site of Nup100p within the NPC could not be identified so far. However, it is considered to bind to the same location within the NPC as Nup116p (Ho et al., 2000). In line with this assumption, Nup100p and Nup145Np were found to bind to Nup116p's binding site on Nup82p in a mutually exclusive manner in vitro (Yoshida et al., 2011).

Deletion of NUP116 was shown to result in morphological changes of the nuclear envelope accompanied by a severe growth delay and temperature sensitivity (Wente and Blobel, 
1993; Wente et al., 1992). These phenotypes could partially be attributed to the concomitant deletion of the conserved Gle2p binding site (GLEBS domain) that intervenes the FG domain of Nup116p (Bailer et al., 1998). Likewise, Nup98 harbors a binding site for Rae1, the vertebrate homolog of Gle2p (Pritchard et al., 1999).

Gle2p is a conserved RNA export factor (Brown et al., 1995; Murphy et al., 1996) and its interaction with Nup116p is crucial for Gle2p in vivo function (Bailer et al., 1998). Notably, Bailer et al. (1998) functionally transplanted the GLEBS domain from Nup116p to Nup100p and therewith rescued an observed Nup116p $\Delta$ GLEBS phenotype. In contrast, transplantation of the GLEBS domain to Nup49p or Seh1p did not rescue the phenotype.

In a Xenopus egg extract system, Nup98 was found to predominantly establish the permeability barrier of vertebrate NPCs (Hülsmann et al., manuscript submitted). Especially, its cohesive FG domain is sufficient to maintain a permeability barrier. Remarkably, replacing the Nup98 FG domain by the FG domains of Nup100p or Nup116p results in functional pores in vitro.

\subsubsection{The Nup82 complex}

The Nup82 subcomplex comprises Nup82p, Nsp1p, Nup116p, and Nup159p (Belgareh et al., 1998; Hurwitz et al., 1998; Ho et al., 2000; Bailer et al., 2000). Dbp5p, a DEADbox RNA helicase involved in termination of mRNA export, associates with the complex by binding to Nup159p (Weirich et al., 2004).

Nup82p is located exclusively at the cytoplasmic side of the NPC (Hurwitz et al., 1998) and seems to serve as a platform to attach the FG Nups Nsp1p, Nup116p, and Nup159p to the NPC scaffold (Yoshida et al., 2011).

\subsubsection{Peripheral FG Nups}

The FG domains of the peripheral FG Nups do not preferentially radiate into the NPC channel. Instead, they constitute filamentous structures observed on both, the nucleoplasmic and the cytoplasmic, sides of the NPC (see figure 1.1).

\section{Cytoplasmic filaments}

Nup159p is an essential protein (Gorsch et al., 1995). It is a component of the Nup82 subcomplex that is thought to form filamentous structure at the cytoplasmic side of the NPC (Belgareh et al., 1998; Hurwitz et al., 1998). It consists of several domains with 
distinct functions. The N-terminal domain functions in mRNA export by tethering the helicase Dbp5p to the NPC (Priore et al., 1997; Weirich et al., 2004). The centrally localized domain is rich in FG motifs (Gorsch et al., 1995). C-terminal of this FG domain is the dynein light chain interacting domain (DID) that binds Dyn2p, which is thought to project the Nup159p FG domain from the structural core of the Nup82 complex (Stelter et al., 2007). Nup159p is anchored to the Nup82 subcomplex via its C-terminal domain that folds into an amphipathic $\alpha$-helix (Priore et al., 1997; Yoshida et al., 2011).

The second FG Nup that localizes exclusively to the cytoplasmic side of the NPC is Nup42p, also known as Rip1p. It was originally identified in a screen as being a yeast target of the HI-Virus protein Rev (Stutz et al., 1995). It is not essential and was proposed to specifically function in the export of heat shock mRNAs (Saavedra et al., 1997), even though the deletion of its FG domain has no effect on mRNA export (Stutz et al., 1997).

\section{Nucleoplasmic filaments}

Nup1p is essential for cell viability, and its over-expression inhibits cell growth (Davis and Fink, 1990). Notably, some yeast strains bear a natural occurring suppressor that renders Nup1p to be non essential (Belanger et al., 1994). Nup1p is anchored to the NPC via its N-terminal domain probably by binding to Nup157p and Nup170p (Kenna et al., 1996). Nup1p and Nup60p were found to localize exclusively to the nucleoplasmic side of the NPC, and together with Nup2p, Mlp1p, and Mlp2p they are thought to form the nuclear basket structure of the yeast NPC (Rout et al., 2000; Kiseleva et al., 2004).

The localization of Nup2p is less defined: It was found to be a nucleo-cytoplasmic shuttling protein that dynamically associates with the nucleoplasmic side of the NPC (Dilworth et al., 2001). It binds the NPC via Nup60p in dependence of the intranuclear concentration of Gsp1p-GTP, the yeast homolog to the vertebrate GTPase Ran (Denning et al., 2001). The FG domains of Nup1p, Nup2p, and Nup60p belong to the "FxF" type FG domains. They were found to be non-essential and can be deleted simultaneously without affecting viability (Strawn et al., 2004). 


\subsection{Models of nucleocytoplasmic transport across the NPC permeability barrier}

The permeability barrier of NPCs excludes inert macromolecules $>40 \mathrm{kDa}$ (Paine et al., 1975 ) or $\geq 5 \mathrm{~nm}$ in diameter (Mohr et al., 2009). At the same time it permits facilitated passage of even very large cargoes, provided these are bound to NTRs (reviewd e.g. by Görlich and Kutay, 1999; Fried and Kutay, 2003). NTRs bind to FG motifs (e.g. Iovine et al., 1995; Radu et al., 1995; Bayliss et al., 2000) and this binding was found to be essential for facilitated NPC passage (Bayliss et al., 1999). Actually, this binding even accelerates NPC passage of the NTR -cargo complex by a factor of up to 1000 compared to the passage of an inert objects of similar size (Ribbeck and Görlich, 2001). It is however not trivial to explain this phenomenon, since a binding should instead cause the retention of the NTR·cargo complex and therefore delay the NPC passage.

In the past years, several models were proposed that try to resolve this paradox.

\subsubsection{The reduction of dimensionality model}

The "reduction of dimensionality" model (Peters, 2005, 2009) assumes that FG domains line the channel of the NPC but leave a free transit way in the middle of the NPC that allows the diffusion of small molecules. However, this channel is so narrow that free transit of molecules with a diameter above a certain limit is restricted. In this model, NTRs act as "ferries" for their bound cargos. They slide along the FG layer of the channel surface by a two dimensional random walk. This sliding is thought to be considerably faster than a three dimensional random diffusion of inert material. This model is well in line with the observation that cells are viable after deletion of more than half of the FG mass (Strawn et al., 2004), assuming that the remaining FG domains are still sufficient to form a thin layer of FG mass but inconsistent with the observed permeability of the NPC. Given that ribosomes with a diameter of $\sim 25 \mathrm{~nm}$ can pass, the central channel cannot be narrower than $25 \mathrm{~nm}$. Such wide channel can however not significantly hinder passage of $5 \mathrm{~nm}$-sized objects.

\subsubsection{The virtual gating model}

The "virtual gate" model (Rout et al., 2000, 2003) assumes that the NPC forms an aqueous central channel that is surrounded by FG domains. This channel is so narrow that molecules larger than the size limit are unlikely to enter. However, FG domains at the 
cytoplasmic and at the nucleoplasmic side of the NPC provide initial docking sites for NTRs, increasing the residence time of NTR - cargo complexes at the entrances to the central channel. This initial docking is thought to increase the probability of the complexes to enter the channel and to subsequently diffuse through it.

Inert macromolecules are repulsed by FG domains from entering the NPC channel whereas small objects can easily lurch through this entropic barrier.

However, the model does not explain how NTR·cargo complexes with a diameter larger than the diameter of the narrow channel can cross the NPC.

\subsubsection{The reverse collapse model}

The "virtual gate" model was extended by the observation that some FG domains collapse from their natively unfolded protein structures into a more collapsed-coil configuration (Lim et al., 2006, 2007). In this "reversible collapse model", NTR·cargo complexes are thought to move across the barrier via contracting FG domains.

However, a recent study did not observe collapse of FG domains in the presence of NTRs (Eisele et al., 2010) and the authors argue that the previously observed collapse can be attributed to the low density of FG domains in the experimental setup. Thus reversible collapse is unlikely to occur in the central region of the NPC (Eisele et al., 2010).

\subsubsection{The selective phase model}

In contrast to the previously described models, the "selective phase" model (Ribbeck and Görlich, 2001; Frey and Görlich, 2007) relies on interactions between FG motifs that crosslink the FG domains into a sieve-like meshwork. And free diffusion of inert molecules is controlled only by the mesh size. NTRs overcome this size limit by binding to FG motifs. The binding results in an opening of the FG meshes. Due to the fact that NTRs harbor multiple binding sites for FG motifs (Bayliss et al., 2000, 2002b; Bednenko et al., 2003; Isgro and Schulten, 2005) they subsequently become a part of the meshwork.

The "selective phase" model is supported by the observation that cohesive FG domains spontaneously form macroscopic hydrogels by creating inter-repeat contacts (Frey et al., 2006; Frey and Görlich, 2009; Milles and Lemke, 2011). The needed concentration for hydrogel formation was found to be in the range of the calculated concentration of FG domains within nuclear pores (Bayliss et al., 1999; Frey et al., 2006).

A macroscopic hydrogel displays permeability properties very similar to those of authentic NPCs. It effectively suppresses the influx of inert macromolecules, while the uptake of 
NTRs occurs so rapidly that it is only limited by the rate of their diffusion to the barrier (Frey and Görlich, 2007). Strikingly, the selectivity of macroscopic hydrogels increase when they are pre-incubated with NTRs (Frey and Görlich, 2009). Additionally, the ability of FG domains to form such hydrogels is conserved during evolution (A. Labokha \& B. Schmidt, personal communication).

Since yeast NPCs contain numerous cohesive FG repeat domains (Patel et al., 2007; Frey and Görlich, 2007, 2009; Yamada et al., 2010), it is plausible to assume that hydrogel formation occurs in authentic NPCs. A recent study directly showed that multivalent cohesion between FG repeats is indeed required (Hülsmann et al., manuscript submitted). However, it is still unclear whether hydrogels mimic the situation in NPCs in vivo.

\subsubsection{The forest model}

Like the "selective phase" model, the recently proposed "forest" model (Yamada et al., 2010) relies on hydrophobic interactions. However, not all FG domains were found to be cohesive (Patel et al., 2007; Yamada et al., 2010). Thus, inter-repeat cohesions might not be the only defining features. Based on measured stoke radii and molecular dynamic simulations, Yamada et al. (2010) proposed the existence of two distinct conformations of FG domains. FG domains that adopt a proposed collapsed coil conformation place the hydrophobic FG motifs in direct proximity to the anchoring domains. These "shrubs" thus line the wall of the NPC channel. In contrast, some FG domains, the "trees", allocate FG motifs to the central part of the channel by partially adopting an extended coil conformation. Only the tops of the "trees" form a hydrogel like structure in the central part of the channel. Here, large objects like ribosomal subunits pass the NPC according to principles described by the "selective phase" model. The "shrubs" act more like an entropic gate, as proposed by the "virtual gate" model. Smaller NTR·cargo complexes preferentially move through this zone.

\subsection{Yeast genetics to study the NPC permeability barrier}

Yeast genetics has been extensively used to study the protein composition of the NPC and the contribution of individual nucleoporins in protein and RNA transport (reviewed by Fabre and Hurt, 1997). Thus the general involvement of FG Nups and their crucial function in nucleo-cytoplasmic transport is generally established (reviewed e.g. by Terry and Wente, 2009). 
Additionally, yeast genetic studies are a useful tool to test predictions of transport models in vivo. As an example, it was suggested that the asymmetric localization of discrete Nups might be in part responsible for conferring directionality of protein import (Ben-Efraim and Gerace, 2001). Addressing this issue, Zeitler and Weis (2004) showed that an asymmetric distribution of FG domains between the nucleoplasmic and the cytoplasmic side of the NPC is not required for bulk protein import in vivo. Even though they genetically swapped the FG domains from Nup1p and Nup159p, protein import was not impaired.

An other yeast genetic study systematically analyzed relationships between all FG domains in the NPC. Strawn et al. (2004) reported the generation of deletion strains deficient in various combinations of FG domains. The authors first defined every FG domain of the NPC and then genomically removed it. All but one possible single FG domain deletions resulted in viable strains. Only deletion of the entire Nup116p FG domain was lethal. Thus, for subsequent steps only the Nup116p FG subdomain was removed which is located C-terminal of the GLEBS domain (figure 1.2).

As a next step, mutants for the centrally localized Nups were combined in all possible pair-wise combinations. Since only the combined deletion of Nup100p and Nup116p FG domains resulted in a non viable strain, a third - and where possible a fourth - deletion was added to each double mutant. Additionally, the authors analyzed specific transport pathways in their mutant strains.

In the end, Strawn et al. came to several conclusions: (i) not all FG domains are functionally equivalent, (ii) the remaining FG domain mass is insufficient to predict viability and transport efficiency, and (iii) NTRs have different requirements for individual FG domains.

For the generation of their multi FG domain deletion strains, Strawn et al. applied a strategy based on a selection marker gene flanked by loxP sites (see figure 1.3 A). After successful integration of this deletion cassette in diploid yeast cells and thereby replacing one allele of the targeted gene, Cre recombinase (Sauer, 1987) was expressed. The recombinase activity led to excision of the selection marker (figure $1.3 \mathrm{C}$ ), allowing its reuse in further rounds of deletion.

Cre-driven recombination of loxP sites is a common technique in mouse genetics (for review see e.g. Sauer, 1998). For this application, loxP sites are targeted to non-coding regions flanking an exon. Normally, targeted exons are chosen in a way that their excision finally results in a frame shift mutation. Thus no functional protein can be produced. The 
(A)

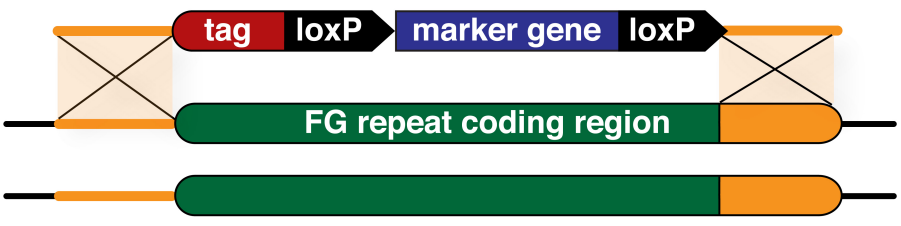

(B)

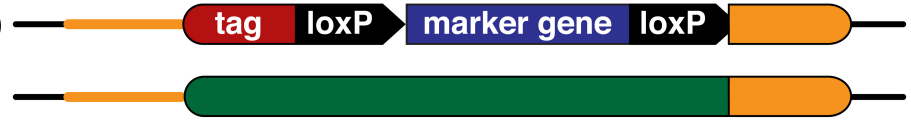

(C)

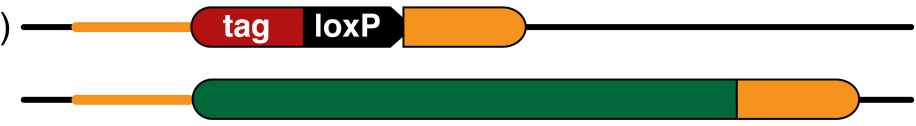

(D) 1. MASMTGGQQMGTSTTLNITSYNVCYTKLLGDIRST

2. CEQKLISEEDLTSTTLNITSYNVCYTKLLGDIRST

3. DYKDDDDKTSTTLNITSYNVCYTKLLGDIRST

Figure 1.3: Genetic strategy to genomically delete coding regions of FG domains using Cre recombinase as applied by Strawn et al. (2004) (A) A deletion cassette harboring a selection marker (blue) flanked by loxP sites (black) is targeted to the coding region of a specific FG domain (green) by homologous DNA regions (orange). Note that coding regions are depicted as rounded boxes. (B) Integration of the deletion cassette in diploid cells results in the substitution of the FG domain coding sequence by the deletion cassette on one allele. A wild type protein can still be expressed from the remaining second allele. (C) After expression of Cre recombinase, the selection marker is excised. DNA coding for an $\mathrm{N}$-terminal tag and one loxP site persists in the coding sequence, being in frame with the remaining protein coding sequence (orange box). (D) Expressed amino acid sequences from the integrated tag (red) followed by the amino acids that are coded by the loxP sequence (black) for three different deletion cassettes including different tags: 1 . $t 7$ tag, 2. myc tag 3. flag tag 
Cre loxP system was also applied in yeast genetics to completely delete a gene.

In contrast to the previously described approaches, Strawn et al. only targeted a part of the gene. As a consequence, the remaining loxP site became part of the coding region that was later on translated into the encoded protein. In combination with an additionally integrated N-terminal tag this led to the attachment of 32-35 additional amino acids to the remaining domain of the protein (figure $1.3 \mathrm{D}$ ).

\subsection{Aim of this study}

FG hydrogels reproduce the permeability properties of NPCs almost perfectly. However, it is still unclear whether FG hydrogels form in authentic NPCs and are required for in vivo NPC function.

The obvious solution to address this issue would be to introduce point mutations that change the cohesive FG domains into non-cohesive ones that do not form hydrogels in vitro and test the effects of such mutations on viability and transport. However, this requires more than point mutations, since FG domains consist of many divergent repeat motifs. In addition, not only in the sequences of the repeat motifs differ within a given FG domain but also the sequences of the intervening spacers. Likewise, FG domains differ from one nucleoporin to another. And in total yeast possesses 11 different Nups with FG domains (Rout et al., 2000).

However, the significance of most of these observed differences among FG domains for the functionality of the permeability barrier in vivo so far remained unclear.

The focus of my PhD work has been to elucidate which FG domain features are essential for permeability barrier function in vivo. Therefore, the immediate aim of this project has been to establish a genetic assay that allows us to test individual FG domains, motifs or artificial FG domains for their ability to support NPC function and viability. We want to know which features of FG domains are important for NPC selectivity and, concomitant with that, for viability.

By relocating FG domains from one anchor point to an other one and monitoring the viability of resulting cells, we want to answer questions such as whether the anchoring and thereby the relative position of a domain within the NPC influences its overall functionality and whether FG domains are functionally equivalent.

If we find a strains that relies on the presence of a given FG domain for viability, we will change the length, the NTR binding sites or the cohesiveness of the FG domain and test 
whether this influences the viability of the strain. By doing so we will answer questions about what FG domain features are important for NPC functionality.

Overall, we want to be able to extrapolate determinant features of FG domains, beside cohesiveness and NTR binding, for permeability barrier function. 


\section{Results}

FG domains have been known for being the building blocks of the NPC permeability barrier for many years. In vitro studies accumulated data about properties of individual FG domains from a variety of organisms and allowed the proposal of models for barrier function. However, information about the in vivo contribution of individual FG domains are still insufficient and experimental systems to test predictions from proposed models for in vivo functionality are missing.

We set out to establish such a system that not only allows the analysis of an in vivo permeability barrier but also offers the potential to simultaneously manipulate components of the barrier in vivo.

The yeast Saccharomyces cerevisiae was chosen as a model system since (i) methods for its genetic manipulations are well established, (ii) it is one of the best studied model organism in cell biology, and (iii) the FG nucleporins of $S$. cerevisiae belong to a group of biochemically well characterized FG Nups.

As a first step, we wanted to establish a genetic assay that allows to evaluate the importance of a given FG domain for viability. For this assay, a mutant strain with a maximal number of genomically deleted FG domains is needed. The according anchor domains (that anchor the FG Nups to the rigid NPC scaffold) should not be impaired in this strain. Deletion of a next FG Nup should then be lethal and supplying the anchor domain of this Nup alone should not be sufficient to restore viability. In such a strain, the in vivo functionality of any FG domain can be assayed by transforming a plasmid that codes for a fusion of this FG domain with the anchor domain of the completely deleted Nup.

Based on the previous observation that a triple deletion of the FG domains of Nup49p, Nup57p, and Nsp1p is lethal and that viability can be restored with either FG domains of the Nup49p, Nup57p, or Nsp1p (Strawn et al., 2004), these combinations of deletions were chosen as starting points. 


\subsection{The combined deletions of FG domains from Nsp1p, Nup49p, and Nup57p are not lethal}

Lethality in the previously created triple $\Delta \mathrm{FG}$ mutant strain (Strawn et al., 2004) is rescued by a Nsp1p wild type coding vector. In principal, this strain could already be used to test FG domains fused to the Nsp1p anchor domain. However, problematic in that strain is the residual existence of a genomically encoded Nsp1p anchor domain. With this genomic copy there are in total three genetic sources of Nsp1p anchor domains: (i) the genomically coded one (Nsp1p anchor), (ii) Nsp1p wild type originating from the rescuing vector, and (iii) Nsp1p anchor domain fused to the FG domain to be tested (Test ${ }^{\mathrm{FG}}-\mathrm{Nsp} 1 \mathrm{p}^{\text {anchor }}$ ). The composition of the NPC at a given time is thus not precisely controllable.

The obvious solution for the generation of a Nsp1p, Nup49p, Nup57p triple FG domain deletion strain was to completely delete one of these three FG nucleoporins in a background where the two other corresponding FG domains were already genomically deleted.

\subsubsection{Remaining scar domains result in a deleterious effect}

Strawn et al. (2004) reported the generation of several combinations of double FG domain deletion strains. For the generation of their strains with multi FG domain deletions, the authors applied a recombinase strategy. The used recombinase recognition site remained in the amino acid coding region of the anchor domain. In combination with an additionally integrated N-terminal tag this resulted in the attachment of 32-35 amino acids to the anchor domain (see section 1.3 and figure $1.3 \mathrm{D}$ ).

Three new strains with combined FG domain deletions of Nsp1p, Nup49p, and Nup57p including one complete gene deletion, respectively, were generated on the basis of these strains. Due to the recombinase based deletion strategy of parental strains, the resulting strains have the following genotypes:

$$
\begin{aligned}
& \Delta \text { nup } 49 \text { flag-loxP-nsp } 1 \Delta F G \text { myc-loxP-nup } 57 \Delta F G+N U P 49 \text { URA3 CEN } \\
& \Delta \text { nup57 t7-loxP-nup } 49 \Delta F G \text { flag-loxP-nsp1 } \Delta F G+N U P 57 \text { URA3 CEN } \\
& \Delta \text { sp1 tr-loxP-nup } 49 \Delta F G \text { myc-loxP-nup57DFG + NSP1 URA3 CEN }
\end{aligned}
$$

The expected lethality caused by the complete deletion of one of the Nups is rescued by a vector coding the corresponding wild type Nup and the URA3 selection marker gene. This allows the use of the FOA based complementation assay (4.2.2.9). With that assay 
we tested whether the anchor domain of the completely deleted Nup alone is sufficient to rescue loss of the wild type coding rescue vector.

transformed with LEU2 vector coding FOA

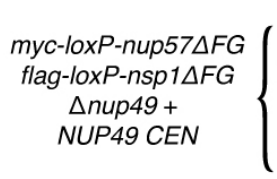
t7-loxP-nup $49 \Delta F G$
flag-loxP-nsp1 $1 F G$
Dnup57+
NUP57 CEN

t7-IoxP-nup49 $\triangle F G$ myc-loxP-nup57 $\triangle F G$ $\triangle n s p 1+$ NSP1 CEN
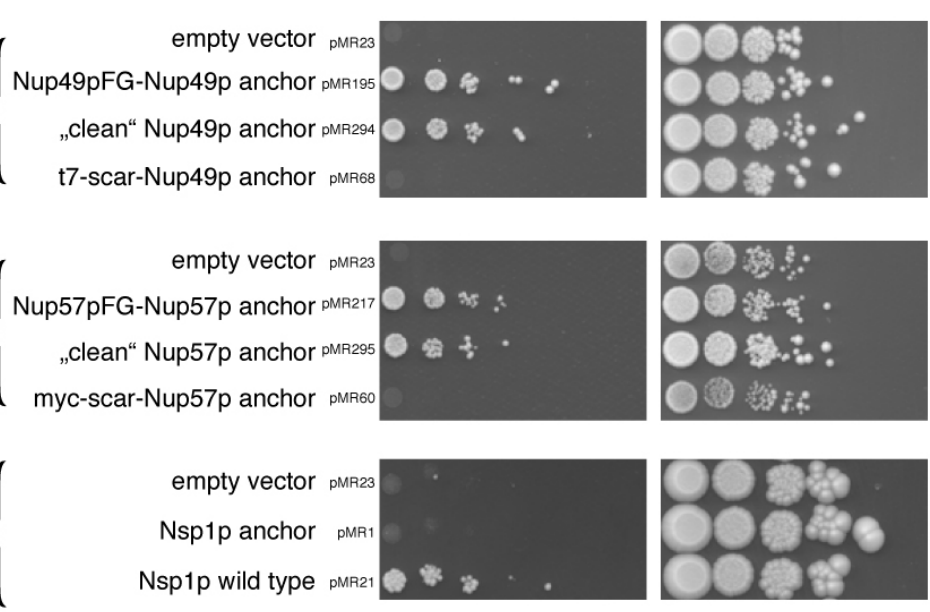

Leu
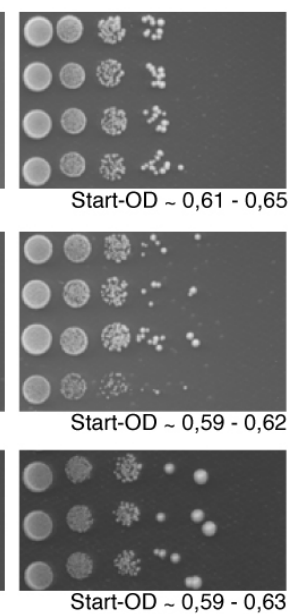

Figure 2.1: Effect of a deletion scar: Plasmids encoding the specified proteins were transformed into strains with genetic backgrounds as indicated (from top to bottom: YMR27, YMR28, YMR26). Additionally the strains contained a URA3 harboring plasmid that encoded an essential wild type protein. In this so-called FOA Assay, the ability of the encoded fusion protein to functionally replace the according wild type protein is assayed by monitoring cell growth on FOA containing plates. If the fusion protein cannot functionally replace the wild type protein, cells depend on the presence of the wild type coding plasmid. However, in such a case cell growth is prevented since the URA3 gene product converts FOA in an toxic compound. For details see section 4.2.2.9. Cells were incubated for 5 days (top and middle panel) or 8 days (bottom panel) at $25^{\circ} \mathrm{C}$, respectively. The exact amino acid composition of the fusion proteins can be found in section 4.1.10.

In contrast to published data (Strawn et al., 2004), it turned out that already the Nup49p anchor domain alone was able to complement the loss of the NUP 49 gene in the nsp $1 \triangle F G$ nup 57 $\triangle F G$ background (see figure 2.1 "clean Nup49p anchor"-construct). Likewise the Nup57p anchor domain complemented for the loss of the $N U P 57$ gene in a nsp1 $A F G$ nup $49 \Delta F G$ background. Only the Nsp1p anchor domain, as previously reported, was not sufficient to complement the loss of the NSP1 gene in a nup $49 \Delta F G$ nup $57 \Delta F G$ background.

We could reproduce the reported lethal phenotype, when we mimicked the exact coding region of the published triple FG deletion strains including the genetic "scar" that originated from the recombinase based deletion strategy (see constructs "t7-scar-Nup49p anchor" and "myc-scar-Nup57p anchor" in figure 2.1 respectively). 
The deletion of a single FG domain by recombinase driven excision might not show a different phenotype compared to a "clean" deletion (Strawn et al., 2004). But unexpectedly, the combination of deletions created by this method finally results in a dominant negative effect.

To avoid such a artificial effect, we had to start from scratch to generate strains with multiple clean FG domain deletions. In such strains a generated phenotype should only show the effect due to the loss of the FG domains.

\subsection{S. cerevisiae tolerates more FG domain deletions than expected}

We focused on deleting the FG domains of Nups that are thought to be centrally localized within the NPC, namely Nsp1p, Nup49p, Nup57p, Nup100p, Nup116p, and Nup145Np, in order to efficiently weaken the NPCs permeability barrier. For genomic deletions, we defined the respective FG domains as follows:

Nsp1p FG repeat domain amino acid 3-602

Nup49p FG repeat domain amino acid 2-236

Nup57p FG repeat domain amino acid 2-223

Nup100p FG repeat domain amino acid 2-576

Nup116p FG repeat domain amino acid 2-714

Nup145Np FG repeat domain amino acid 10-209

Applying the strategies described in 4.2.2.4, we obtained several viable strains carrying combinations of FG domain deletions that previously had been described as lethal (Strawn et al., 2004):

$$
\begin{aligned}
& \text { nsp } 1 \Delta F G \text { nup } 49 \Delta F G \text { nup } 57 \Delta F G \\
& \text { nsp } 1 \Delta F G \text { nup } 49 \Delta F G \text { nup } 145 \Delta F G \\
& \text { nsp } 1 \Delta F G \text { nup } 57 \Delta F G \text { nup } 145 \Delta F G \\
& \text { nup } 49 \Delta F G \text { nup } 57 \Delta F G \text { nup } 145 \Delta F G \\
& \text { nsp } 1 \Delta F G \text { nup } 49 \Delta F G \text { nup } 57 \Delta F G \text { nup } 100 \Delta F G \\
& \text { nsp } 1 \Delta F G \text { nup } 49 \Delta F G \text { nup } 100 \Delta F G \text { nup } 145 \Delta F G
\end{aligned}
$$

All strains generated in this study are summarized in table 4.1, page 65 .

Even though some triple $\Delta \mathrm{FG}$ mutant strains generated by the recombinase strategy do 


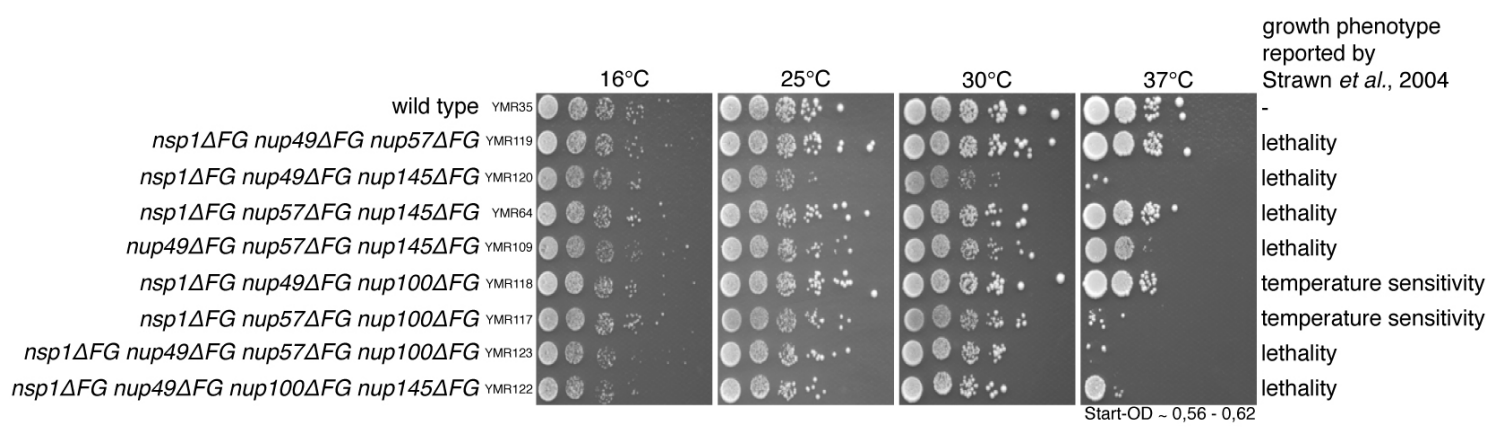

Figure 2.2: Temperature sensitivity assay of multiple $\Delta$ FG mutant strains: Multiple FG domain deletion strains were subjected to a temperature sensitivity assay as described in 4.2.2.10. Cells were allowed to grow for 5 days at $25^{\circ} \mathrm{C}, 30^{\circ} \mathrm{C}$, and $37^{\circ} \mathrm{C}$ and for 8 days at $16^{\circ} \mathrm{C}$. For comparison, the growth phenotypes reported by Strawn et al. (2004) are summarized.

not show a lethal phenotype, they were shown to be temperature sensitive at $37^{\circ} \mathrm{C}$ (Strawn et al., 2004). To test if strains with multiple clean FG domain deletions share such a temperature sensitive phenotype, all strains with three or more FG domain deletions generated in this study where subjected to a temperature sensitivity assay (figure 2.2).

Cold sensitivity or reduced growth at $30^{\circ} \mathrm{C}$ were not observed for any of the strains. Only two of the triple $\Delta \mathrm{FG}$ mutants exhibited reduced growth at $37^{\circ} \mathrm{C}$. This phenotype was previously reported (Strawn et al., 2004) for the nsp $1 \Delta F G$ nup $57 \Delta F G$ nup $100 \Delta F G$ strain. Hence, no difference caused by the scar domains could be observed here.

Remarkably, the quadruple $\Delta \mathrm{FG}$ mutant strain nsp $1 \Delta F G$ nup $49 \Delta F G$ nup $100 \Delta F G$ nup $145 \Delta F G$ only showed reduced growth at $37^{\circ} \mathrm{C}$ even though only the Nup57p- and Nup116p FG domains remain in the central part of the NPC. The nsp $1 \Delta F G$ nup $49 \Delta F G$ nup $57 \Delta F G$ nup $100 \Delta F G$ mutant failed to grow at $37^{\circ} \mathrm{C}$.

Obviously, S. cerevisiae tolerates more FG domain deletions than previously reported (Strawn et al., 2004).

\subsection{Multi $\Delta F G$ mutant strains can be used to assay in vivo functionality of FG domains at a given NPC anchor point}

As demonstrated in the previous chapter, we already massively reduced the number of FG domains, but the resulting cells showed no severe phenotype. It appears that the 
permeability barrier is either built up in a highly redundant manner or the really crucial constituents of the barrier have not been affected so far.

\subsubsection{FG domains at Nup98 homologous anchor position are essential}

Experiments by Hülsmann et al. using a Xenopus egg extract system strongly support the idea that the main component of the vertebrate permeability barrier is the FG domain of Nup98 (manuscript submitted). As summarized in the introduction, there are three Nup98 homologous proteins in S. cerevisiae, namely Nup100p, Nup116p, and Nup145Np. A deeper insight into the functions of FG domains from Nup98 homologs in yeast required simultaneous genetic manipulation of all of these FG nucleoporins.

\subsubsection{The GLEBS domain can be functionally transplanted to Nsp1p}

The investigation of Nup116p FG repeat domain function is hampered by the presence of the GLEBS domain, which intervenes the FG domain of Nup116p. Deleting the complete FG domain including the GLEBS domain results in a loss of NPC function (Iovine et al., 1995). And removing just the FG regions of the domain and thereby relocating the GLEBS domain directly to the N-terminus of the anchor domain was also lethal (see figure $2.4 \mathrm{~A}$, upper part).

Bailer et al. (1998) successfully transplanted the GLEBS domain from Nup116p to Nup100p, which rescued the phenotype caused by the removal of the GLEBS domain from Nup116p. However, this transplantation would not ease the genetic analysis of FG domain functions of yeast Nup98 homologs, since Nup100p is one these homologs.

To gain genetic access to all Nup98 homolog FG domains, we tested the effect of transplanting the GLEBS domain to Nsp1p.

Nsp1p was chosen for two reasons: First, like Nup116p, Nsp1p places a relatively large FG domain to the central part of the NPC so that the GLEBS domain would be positioned into an environment that should mimic its natural surrounding. Second, the Nsp1p FG domain is the only FG domain in the central part of the NPC that does not belong to the "GLFG" type of FG domains. To specifically analyse contribution of "GLFG" type FG domains to NPC function it would be crucial to have genetic access to all of the "GLFG" type Nups. Thus, if transplantation of the GLEBS domain to Nsp1p does not effect its function, reduction of FG mass contributed by "GLFG" type FG domains is possible. Transplantation of the GLEBS domain from Nup116p to Nsp1p has to meet two demands: 
2.3 Multi $\Delta \mathrm{FG}$ mutant strains can be used to assay in vivo functionality of $\mathrm{FG}$ domains at a given NPC anchor point

It should not interfere with normal Nsp1p function and it should enable normal GLEBS domain activity.

\section{Nsp1-GLEBSp fusion does not interfere with Nsp1p function}

We tested the effect of an Nsp1-GLEBSp fusion construct (figure 2.3 C) on Nsp1p function in two genetic backgrounds: (i) $\Delta n s p 1$ (YMR54) and (ii) $\Delta n s p 1$ tr-loxP-nup $49 \Delta F G$ myc-loxP-nup57 $\triangle F G$ (YMR26).

In a $\Delta n s p 1$ background (YMR54) no obvious phenotype could be detected by a temperature sensitivity assay (figure $2.3 \mathrm{~A}$ ). Cells grew nearly equally well at all temperatures tested with either the wild type Nsp1p, the Nsp1p anchor domain only, or the Nsp1GLEBSp fusion protein being present. Furthermore we tested the effect of the Nsp1-

(A)

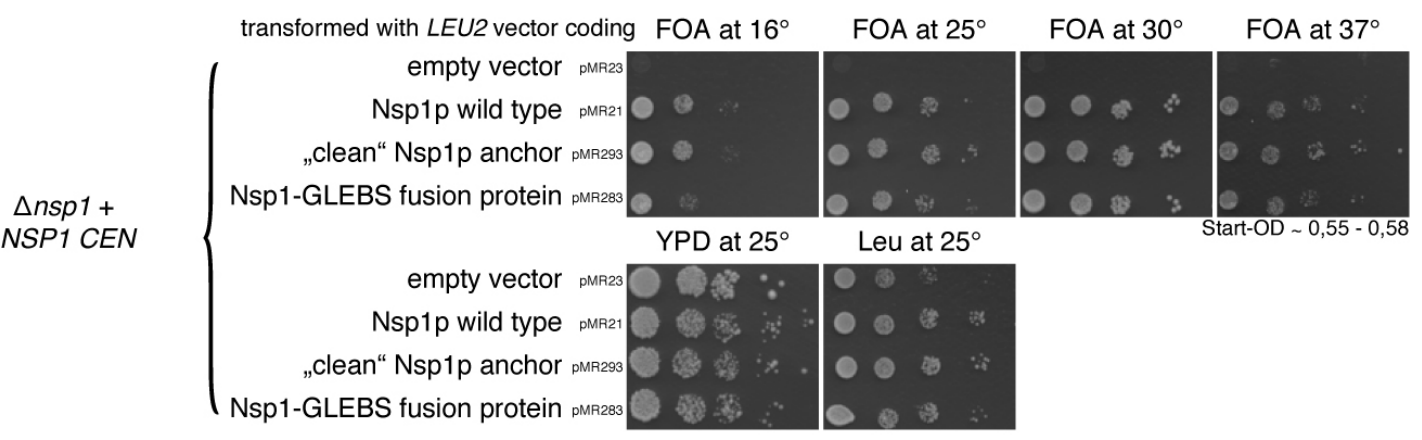

(B)

transformed with LEU2 vector coding FOA
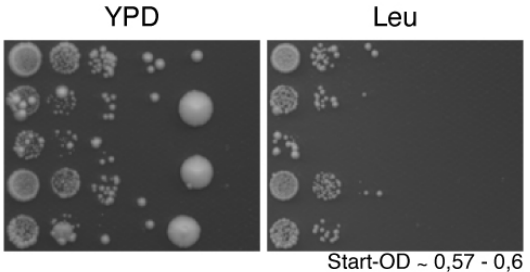

(C)

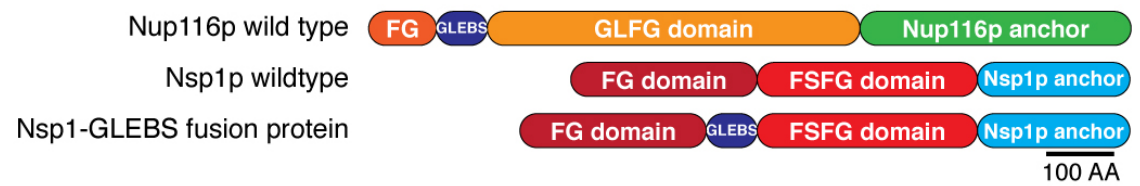

Figure 2.3: Nsp1-GLEBSp fusion does not interfere with Nsp1p function: Constructs were transformed into strains with genetic backgrounds as indicated (panel A: YMR54, panel B YMR26). The ability of the transformed constructs to complement the loss of an Nsp1p wild type protein was monitored by an FOA Assay. (A) Cells on FOA plates were grown for 3 days at $23^{\circ} \mathrm{C}, 30^{\circ} \mathrm{C}$, and $37^{\circ} \mathrm{C}$ and for 8 days at $16^{\circ} \mathrm{C}$. Cells on control plates (YPD, Leu) were grown at $23^{\circ} \mathrm{C}$. Additional controls grown at $16^{\circ} \mathrm{C}$, $30^{\circ} \mathrm{C}$, and $37^{\circ} \mathrm{C}$ behave normal (data not shown). (B) Cells were incubated for 12 days at $25^{\circ} \mathrm{C}$. (C) Schematic representation of Nup116p wild type protein, Nsp1p wild type protein, and the Nsp1-GLEBSp fusion protein. Exact amino acid composition of all fusion proteins can be found in section 4.1.10. 
GLEBSp fusion protein in YMR26. This strain was chosen since its growth is already severely reduced by scar domains present in FG domain deleted Nup49p and Nup57p (figure $1.3 \mathrm{D})$. A possible additional negative effect of the GLEBS-Nsp1 fusion protein should therefore be easily detectable.

After 12 days of incubation on FOA, comparable growth behavior was observed both with wild type Nsp1p and with the Nsp1-GLEBSp fusion protein (figure 2.3 B).

No phenotype of the Nsp1-GLEBSp fusion protein could be detected. So we concluded that the GLEBS domain does not interfere with Nsp1p function in vivo.

\section{GLEBS domain is functional on Nsp1p}

In a next step, we integrated the coding sequence of the Nsp1-GLEBSp fusion protein at the genomic locus of NSP1. Then phenotypes of a NUP116 deletion in a wild type background versus a background with integrated NSP1-GLEBS fusion construct were compared. By doing so, we evaluated if the GLEBS domain is functional when transplanted to Nsp1p (figure 2.4).

As expected, the loss of Nup116p in a wild type background resulted in lethality and could not be complemented by the presence of Nup116p anchor domain or the GLEBS domain directly fused to the anchor domain alone (figure $2.4 \mathrm{~A}$, upper part). In contrast, in an NSP1-GLEBS fusion background, cells were viable after the loss of wild type Nup116p (figure $2.4 \mathrm{~A}$, lower part). In this background NUP116 is no longer essential.

These results indicate the functionality of the GLEBS domain after transplantation to Nsp1p. Additionally we demonstrated that NUP116 becomes dispensable after transplantation. 
(A)

transformed with LEU2 vector coding

FOA

YPD

Leu

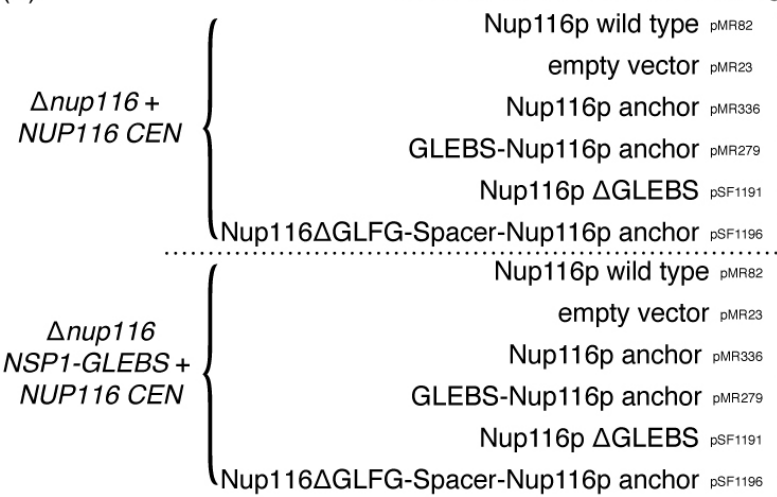

(B)

Nup116p wild type
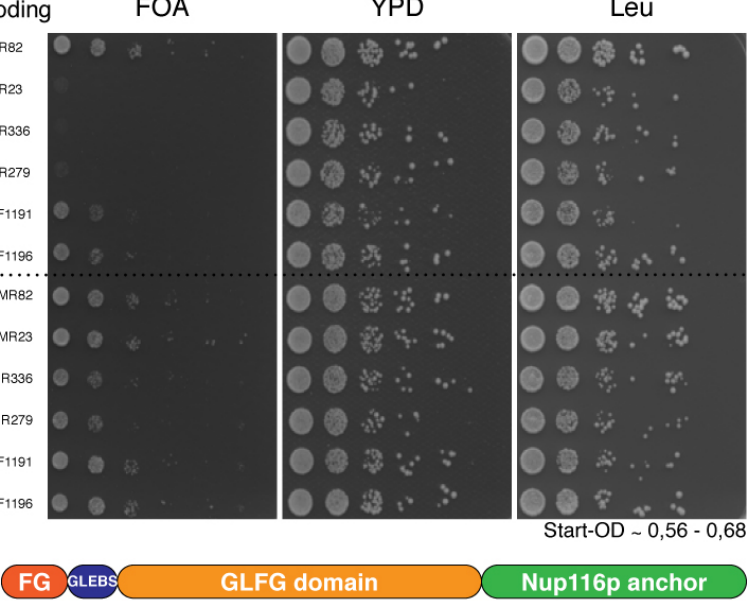

Nup116p anchor GLFG domain

GLEBS-Nup116p anchor

Nup116p $\Delta$ GLEBS

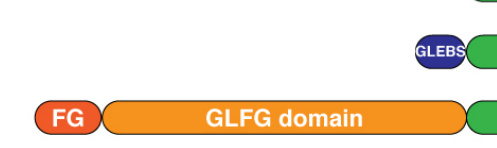

Nup116p anchor

Nup116 $\Delta$ GLFG-Spacer-Nup116p anchor

Figure 2.4: Nup116p becomes dispensable after GLEBS transplantation: $(A)$ Indicated constructs were transformed into YMR58 (upper part) and YMR73 (lower part). The ability of the transformed constructs to complement the loss of Nup116p wild type protein was monitored by an FOA Assay. Cells were incubated for 3 days at $25^{\circ} \mathrm{C}$. The exact amino acid composition of fusion proteins can be found in section 4.1.10. (B) Schematic representation of Nup116p mutant proteins. The spacer sequence consists of 16 repeats of the following amino acid sequence: NSGSGTGSSG. 
2.3 Multi $\Delta F G$ mutant strains can be used to assay in vivo functionality of $F G$ domains at a given

\section{Resulting strains}

Transplantation of Nup116p's GLEBS domain into the Nsp1p FG domain led to dispensability of Nup116p. Thus the genetic analysis of Nup98 homologs in S. cerevisiae is no longer hindered by the presence of the GLEBS domain on Nup116p.

We used the transplantation background to generate the following $\Delta \mathrm{FG}$ mutants:

$$
\begin{aligned}
& \text { nup } 100 \Delta F G \\
& \text { nup } 145 \Delta F G \\
& \text { nup } 100 \Delta F G \text { nup } 145 \Delta F G \\
& \text { nup } 116 \Delta F G \text { nup } 145 \Delta F G
\end{aligned}
$$

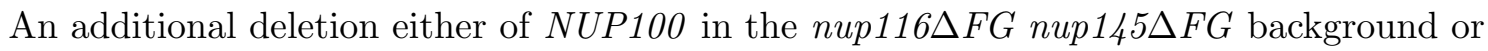
of NUP116 in the nup $100 \triangle F G$ background resulted in lethality that could not be rescued by the presence of the corresponding anchor domain alone (figure 2.5). Effects of other additional deletions are not tested so far.

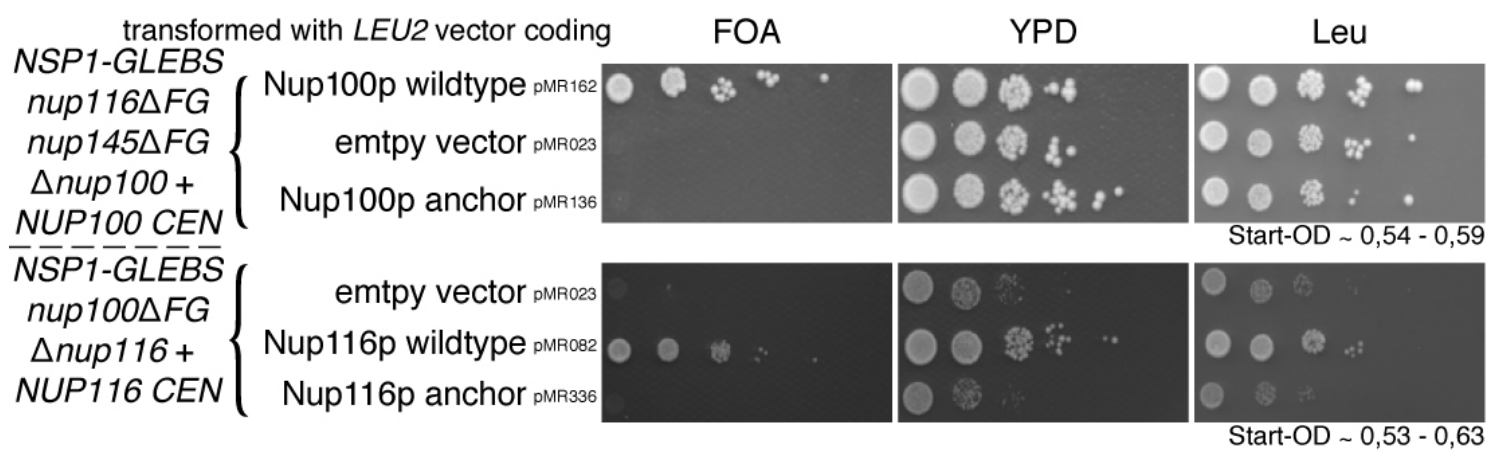

Figure 2.5: Effect of Nup100p and Nup116p deletion: Constructs were transformed into strains with genetic backgrounds as indicated (upper panel YMR128, lower panel YMR127). The ability of the transformed constructs to complement the loss of an according wild type protein was monitored by an FOA Assay. Cells were incubated for 5 days (YMR128) or 2 days (YMR127) at $25^{\circ} \mathrm{C}$, respectively. The exact amino acid composition of the fusion proteins can be found in section 4.1.10.

\subsubsection{Only Nup116p FG domains can functionally replace the Nup100p FG domain}

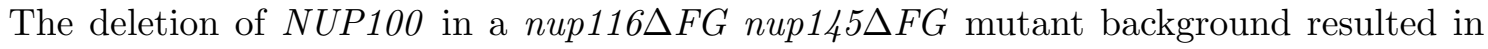
lethality. This phenotype was rescued by plasmid coded Nup100p wild type protein. And replacing Nup100p only by the Nup100p anchor domain did not rescue lethality (figure 2.5). Obviously, viability was only maintained when Nup100p FG domain was present. 
This genetic background offered for the first time the possibility to assay the effect of changing a given FG domain on viability.

With more than 570 amino acids, Nup100p FG domain is the third longest FG domain (after the FG domains of Nup116p and Nsp1p) in S. cerevisiae. To test whether the entire FG domain of Nup100p is essential for viability in this mutant background, shortened mutants of the FG domain were tested (figure 2.6).

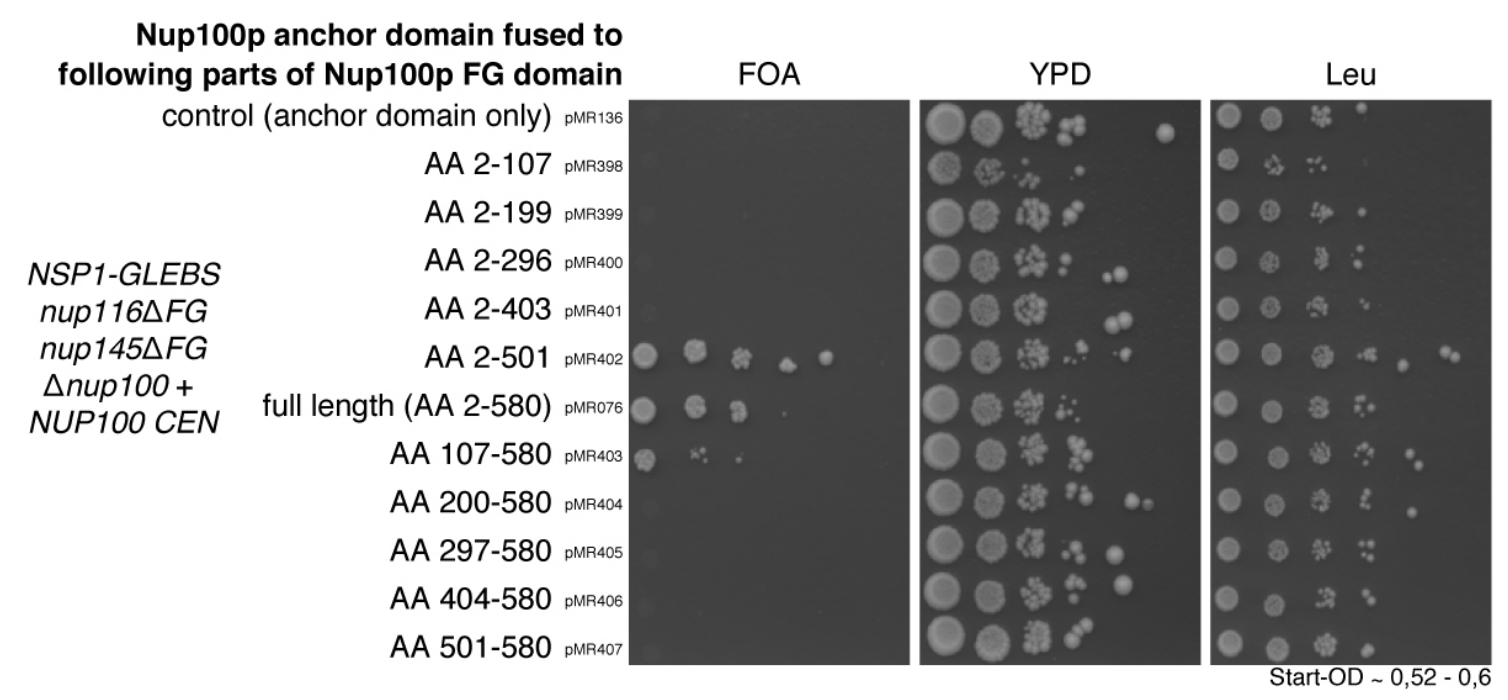

Figure 2.6: Effect of a shortened Nup100p FG domain: Nup100p FG domain was nearly equally divided into five parts. To generate mutant constructs, Nup100p's FG domain was shortened either from the $\mathrm{N}$ - or the C-terminus. The constructs were transformed into YMR128. The ability of the transformed constructs to complement the loss of Nup100p wild type protein was monitored by an FOA Assay. Cells were incubated for 7 days at $25^{\circ} \mathrm{C}$. The exact amino acid composition of the fusion proteins can be found in section 4.1.10.

Most of Nup100p's FG mass was needed to maintain viability. Only a minor fraction of around 100 amino acids could be deleted. It was not important whether the FG domain was shortened from its $\mathrm{N}$ - or C-terminus.

In a next step, we wondered whether FG domains of other Nups can substitute the Nup100 FG domain. Assuming that a certain FG mass at the Nup100p anchor position is needed, only FG domains from Nup116p and Nsp1p would be long enough to compensate for the loss of the Nup100p FG domain. By contrast, if not the quantity of the fused FG domain is relevant, at least some shorter FG domains should result in viable cells. Additionally, this kind of experiment would address the question to what extent variety of FG domains 
is needed.

Thus we assayed the ability of all other endogenous S. cerevisiae FG domains to restore viability in this background when they were genetically fused to the Nup100p anchor domain. Out of the endogenous $S$. cerevisiae FG domains, only the FG domain of Nup116p

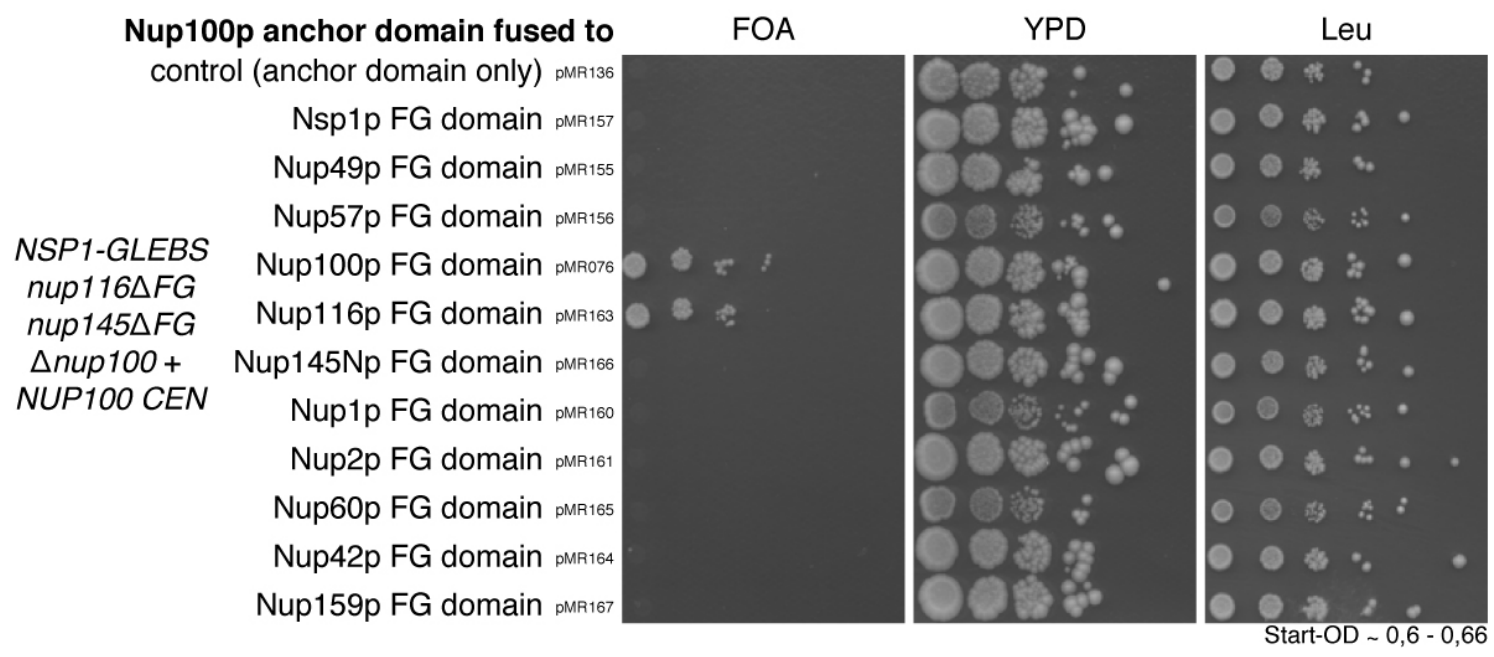

Figure 2.7: Effect of endogenous S. cerevisiae FG domains fused to the Nup100p anchor domain: Indicated FG domains were genetically fused to the Nup100p anchor domain. Their ability to complement the loss of Nup100p wild type was monitored after transformation into YMR128 via FOA Assay. Cells were incubated for 7 days at $25^{\circ} \mathrm{C}$. The exact amino acid composition of the fusion proteins can be found in section 4.1.10.

could replace the FG domain of Nup100p (figure 2.7). Even though the FG domain of

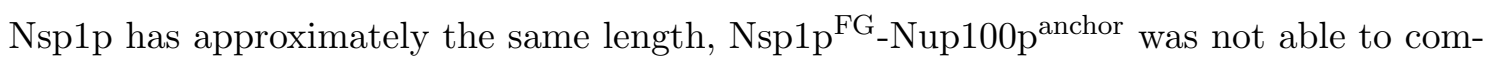
plement the loss of Nup100p wild type. Apparently the type of the FG domain anchored at this position has a great influence on viability.

\subsubsection{Only Nup100p FG domains can functionally replace the Nup116p FG domain}

As described above, we first investigated the in vivo effects of fusing FG domains to the Nup100p anchor position. Only those FG domains originating from Nup100p and Nup116p maintained viability when fused to the Nup100p anchor domain.

To test whether this is a particular peculiarity of the Nup100p anchor domain, we next studied the effects of fusing all endogenous FG domains to the Nup116p anchor domain in the $\Delta$ nup 116 nup $100 \Delta F G$ mutant strain. 


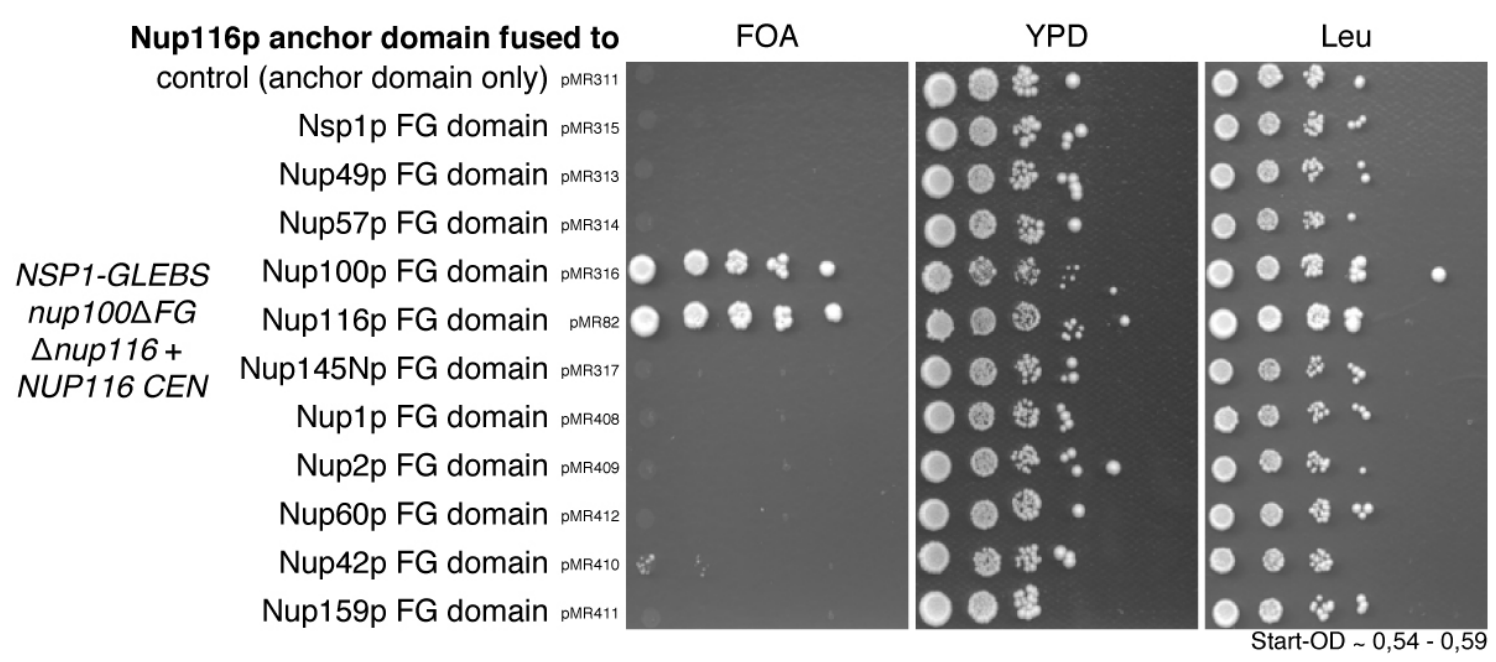

Figure 2.8: Effect of endogenous S. cerevisiae FG domains fused to the Nup116p anchor domain: Indicated FG domains were genetically fused to the Nup116p anchor domain. Their ability to complement the loss of Nup116p wild type was monitored after transformation into YMR127 via FOA Assay. Cells were incubated for 7 days at $25^{\circ} \mathrm{C}$. The exact amino acid composition of the fusion proteins can be found in section 4.1.10.

Again, cells were only viable when either wild type Nup116p or Nup100p ${ }^{F_{-}}$-Nup116 $p^{\text {anchor }}$ were present (figure 2.8).

Surprisingly, the FG domain of Nup145Np, the third S. cerevisiae homolog of vertebrate Nup98, did not rescue viability although its relative amino acid composition is comparable to the Nup100p- and Nup116p FG domain, respectively. One obvious difference is the length of Nup145Np FG domain, which is just 200 amino acids. This is roughly only one third of the length of the Nup100p- or Nup116p FG domain. We therefore assayed whether a multimerized Nup145Np FG domain mutant would enable cell viability.

As shown in figure 2.9, a quadrupled Nup145Np FG domain rescued cell viability after fusion to the anchor domain of Nup100p or Nup116p. But even though viability was achieved with the $4 \times$ Nup145Np FG fusion constructs, cells grew much slower as compared to Nup100p- or Nup116p FG domain fusion constructs.

Apparently, a mere substitution of FG mass is not sufficient to maintain cell viability at this anchor domain. However, the experiment in figure 2.6 argues for the importance of FG mass fused to the Nup100p anchor domain. Taken together these data point to the idea that not only the FG mass is important to constitute a functional permeability barrier but that FG domains have to meet more criteria. 


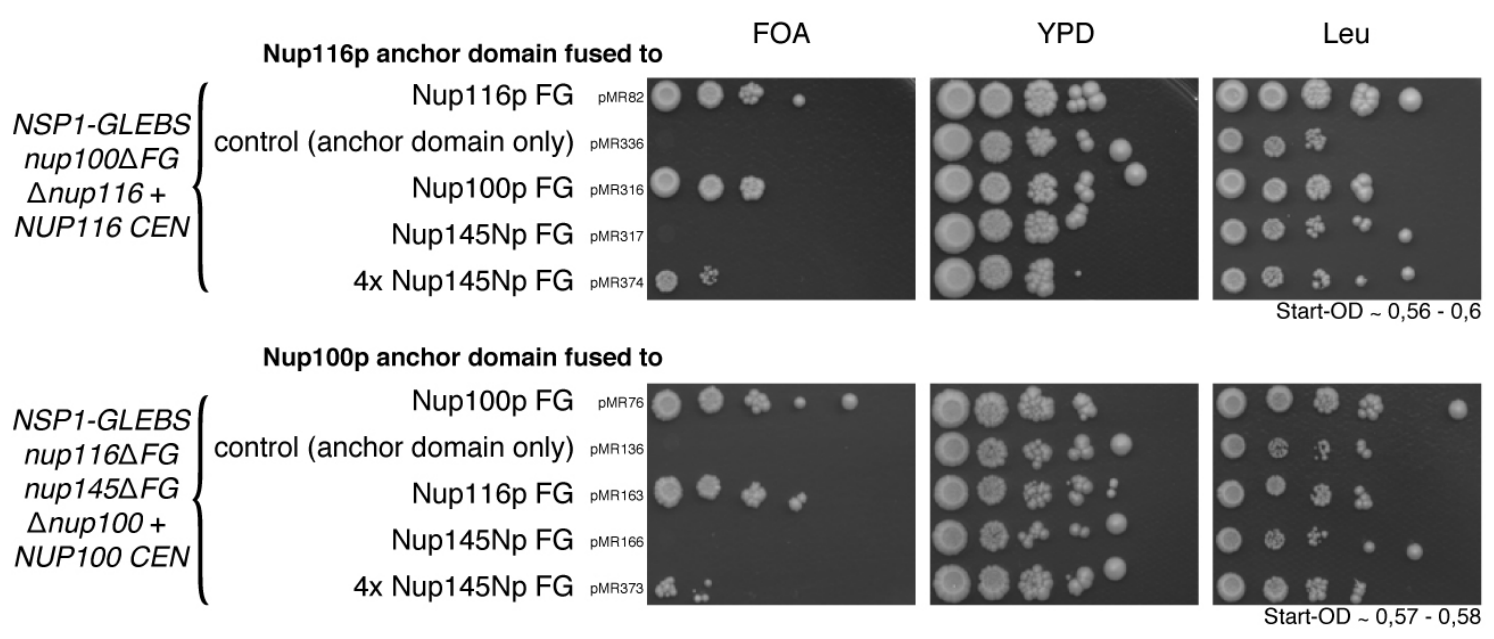

Figure 2.9: Effect of a quadruplicated Nup145N FG domain: Constructs were transformed into strains with genetic backgrounds as indicated (upper panel: YMR127, lower panel: YMR128). The ability of the transformed constructs to complement the loss of an according wild type protein was monitored by an FOA Assay. Cells were incubated for 11 days at $25^{\circ} \mathrm{C}$, respectively. The exact amino acid composition of the fusion proteins can be found in section 4.1.10.

It was shown that cohesive FG domains are important for maintaining the vertebrate permeability barrier (Hülsmann et al., manuscript submitted). Therefore we assume that the presence of a very cohesive FG domain at Nup100p and Nup116p anchor points is likewise crucial for permeability barrier function in yeast.

\subsubsection{Endogenous FG domains can functionally replace the Nsp1p FG domain}

As shown in the previous chapter, the NPC anchoring domains of Nup100p and Nup116p are quite restrictive concerning which anchored FG domains are able to preserve the integrity of NPC permeability barrier as indicated by cell viability. We wondered whether other anchor domains behave in the same manner. Do they only constitute a functional barrier when anchoring their endogenous FG domain, analogous to Nup100p and Nup116p anchor domain? Or can some anchor domains incorporate any FG domain into the NPC such that cell viability is preserved?

To answer these questions, we tested the effects of additional gene deletions in the multi $\triangle F G$ mutant strains. It turned out that the deletion of $N S P 1$ in the nup $49 \Delta F G$ nup $57 \Delta F G$ nup 145 $\triangle F G$ mutant strain was lethal and viability could not be restored by providing the Nsp1p anchor domain alone (figure 2.10). 


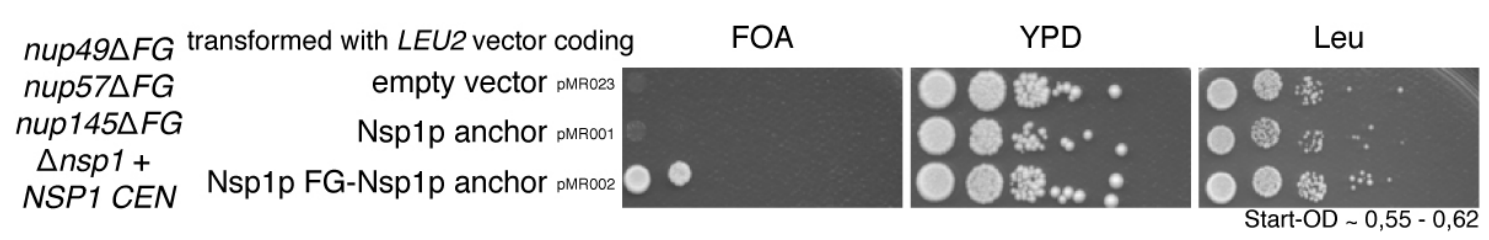

Figure 2.10: FG domains fused to the Nsp1p anchor domain are needed: The ability of the transformed constructs to complement the loss of Nsp1p wild type protein was monitored in YMR113 via FOA Assay. Cells were incubated for 5 days at $25^{\circ} \mathrm{C}$. The exact amino acid composition of fusion proteins can be found in section 4.1.10.

Since the Nsp1p FG domain is only cohesive at its distal end, the Nsp1p anchor domain positions an FG domain within the NPC that is non-cohesive around the anchor point (Ader et al., 2010; Yamada et al., 2010). This distinguishes Nsp1p from Nup100p or Nup116p, since their FG domains are entirely cohesive (Patel et al., 2007; Yamada et al., 2010). Thus, this strain allowed us to study the consequences of fusing various FG domains to an anchor domain which endogenously positions a non-cohesive FG domain within the NPC.

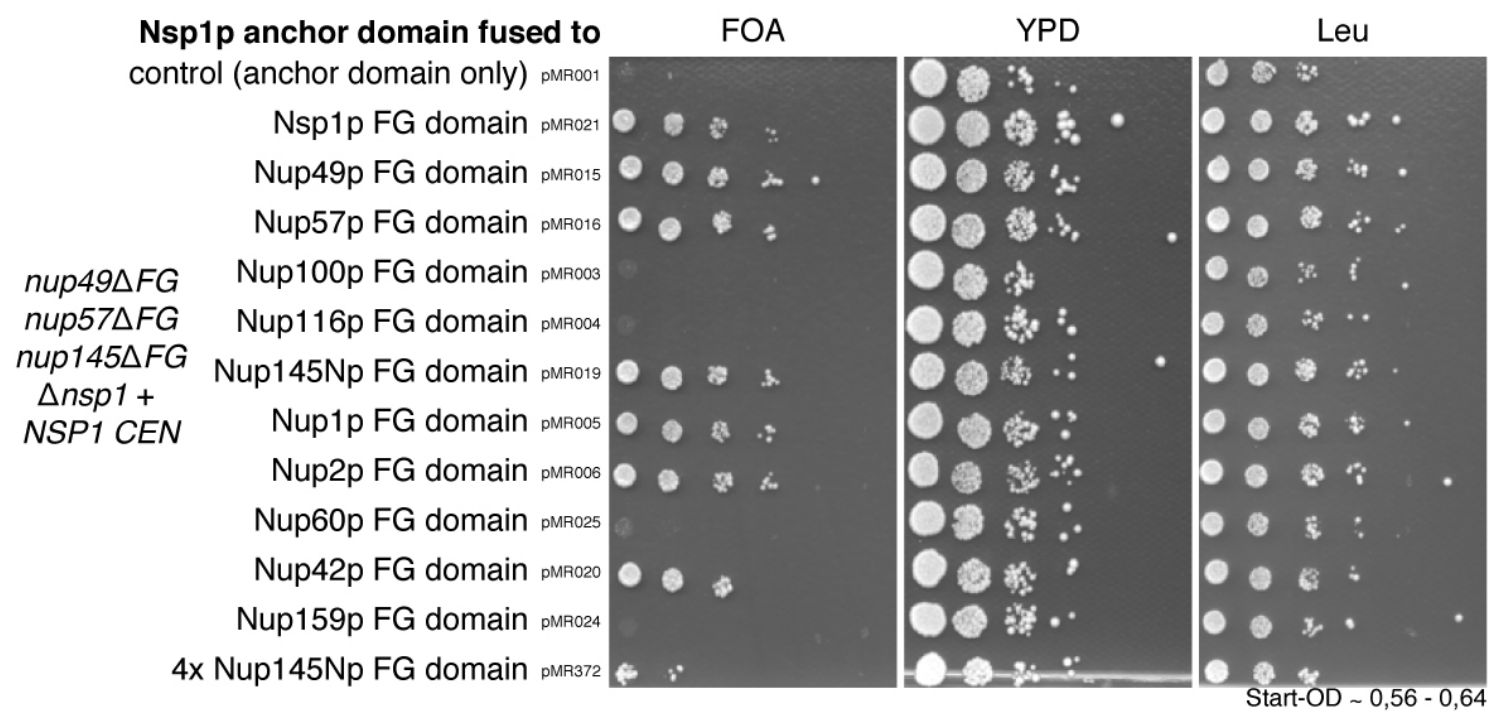

Figure 2.11: Effect of endogenous S. cerevisiae FG domain fused to the Nsp1p anchor domain: Indicated FG domains were genetically fused to the Nsp1p anchor domain. Their ability to complement the loss of Nsp1p wild type was monitored after transformation into YMR113 via FOA Assay. Cells were incubated for 5 days at $25^{\circ} \mathrm{C}$. The exact amino acid composition of fusion proteins can be found in section 4.1.10.

In contrast to the Nup100p- or Nup116p anchor point, a much higher diversity of fused FG domains was functional at the Nsp1p anchor point (figure 2.11), as indicated by cell 
viability. Out of all 11 endogenous $S$. cerevisiae FG domains only those derived from Nup100p, Nup116p, Nup60p, and Nup159p did not rescue cell viability. Irrespective of the FG domains from Nup60p and Nup159p, which cannot functionally replace any tested FG domain, the Nsp1p anchor domain thus tolerates the fusion of a complementary set of FG domains. Even more striking, the fusion protein containing a quadrupled Nup145Np FG domain rescued viability, albeit with a delay in growth. This artificial FG domain seems to be a compromise in terms of supporting viability between the two complementary groups of FG domains.

Obviously the permeability barrier can tolerate a remarkable degree of FG domain substitutions. However, a functional substitution, i.e. resulting in viable cells, is not possible at every anchor domain and with every FG domain respectively. With these result we show for the first time that FG domain anchor points are not equivalent.

\subsection{The Nup100p FG domain causes a dominant negative effect at various anchor points}

Nup100p and Nup116p FG domains are important for viability. A simultaneous deletion results in lethality. Furthermore, we showed that no other endogenous FG domain can maintain cell viability when substituting Nup100p or Nup116p FG domain at their anchor domains. Therefore, it is remarkable that they did not rescue viability when fused to the Nsp1p anchor domain. One simple explanation for this effect would be that the fusion constructs are not incorporated into the NPC. Thereby the essential Nsp1p anchor domain would not be present in the pore, resulting in a lethal phenotype. This possibility was tested in the following experiment.

\subsubsection{Nup100p FG domain fusion constructs are incorporated into the NPC}

We generated Citrine labeled fusion constructs to monitor their incorporation into the NPC by confocal microscopy. Citrine is an enhanced version of the yellow fluorescent protein (Griesbeck et al., 2001). Like with the unlabeled constructs, we expected a non viable phenotype after selection for these constructs in the $\Delta$ nsp1 nup $49 \Delta F G$ nup $57 \Delta F G$ nup 145 $\Delta F G$ mutant strain. Thus, the effect of the fusion protein (Nup100p ${ }^{\mathrm{FG}_{-} \text {-Citrine- }}$ $\mathrm{Nsp} 1 \mathrm{p}^{\text {anchor }}$ ) was evaluated in a $\Delta n s p 1$ strain without any further deletions. Nup100 $\mathrm{p}^{\mathrm{FG}}$ Citrine-Nup49p $\mathrm{p}^{\text {anchor }}$ and Nup100 $\mathrm{p}^{\mathrm{FG}}$-Citrine-Nup57p $\mathrm{p}^{\text {anchor }}$ chimeric proteins were additionally tested in respective deletion strains (figure $2.12 \mathrm{~A}$ ). 
Though fusion of the Nup100p FG domain to the anchor domains resulted in a decreased cell growth, cells were viable. This argues already for a correct insertion of fusion proteins into the NPC. To further prove their incorporation, fluorescence was monitored by confocal microscopy (figure $2.12 \mathrm{~B}$ ).

Clearly, rim staining was observed for all constructs, indicating their incorporation into the NPC. Nevertheless, we cannot fully exclude the possibility that fusion proteins only attached to the nuclear envelope or sticked to the NPC without being incorporated. 
(A)

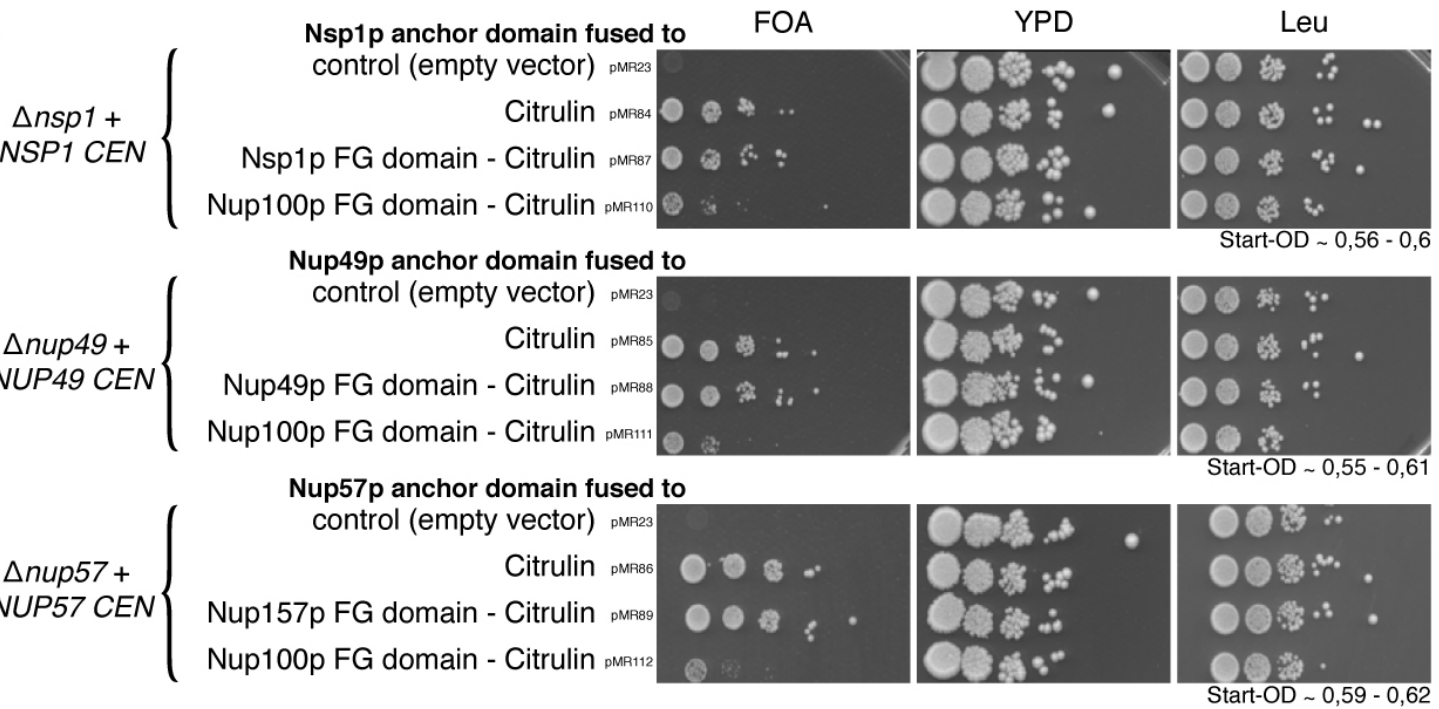

(B)
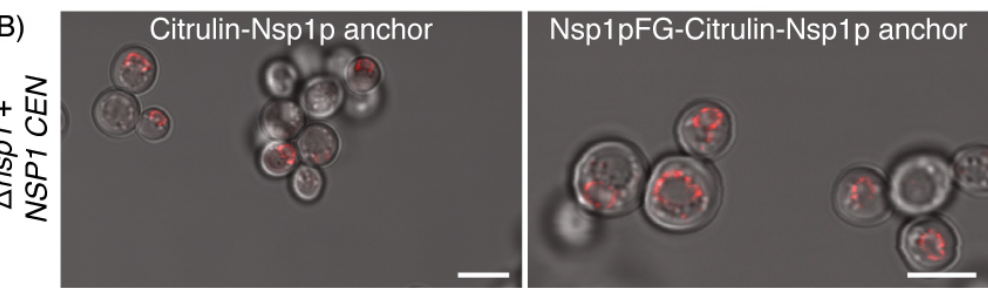

Nup100pFG-Citrulin-Nsp1p anchor
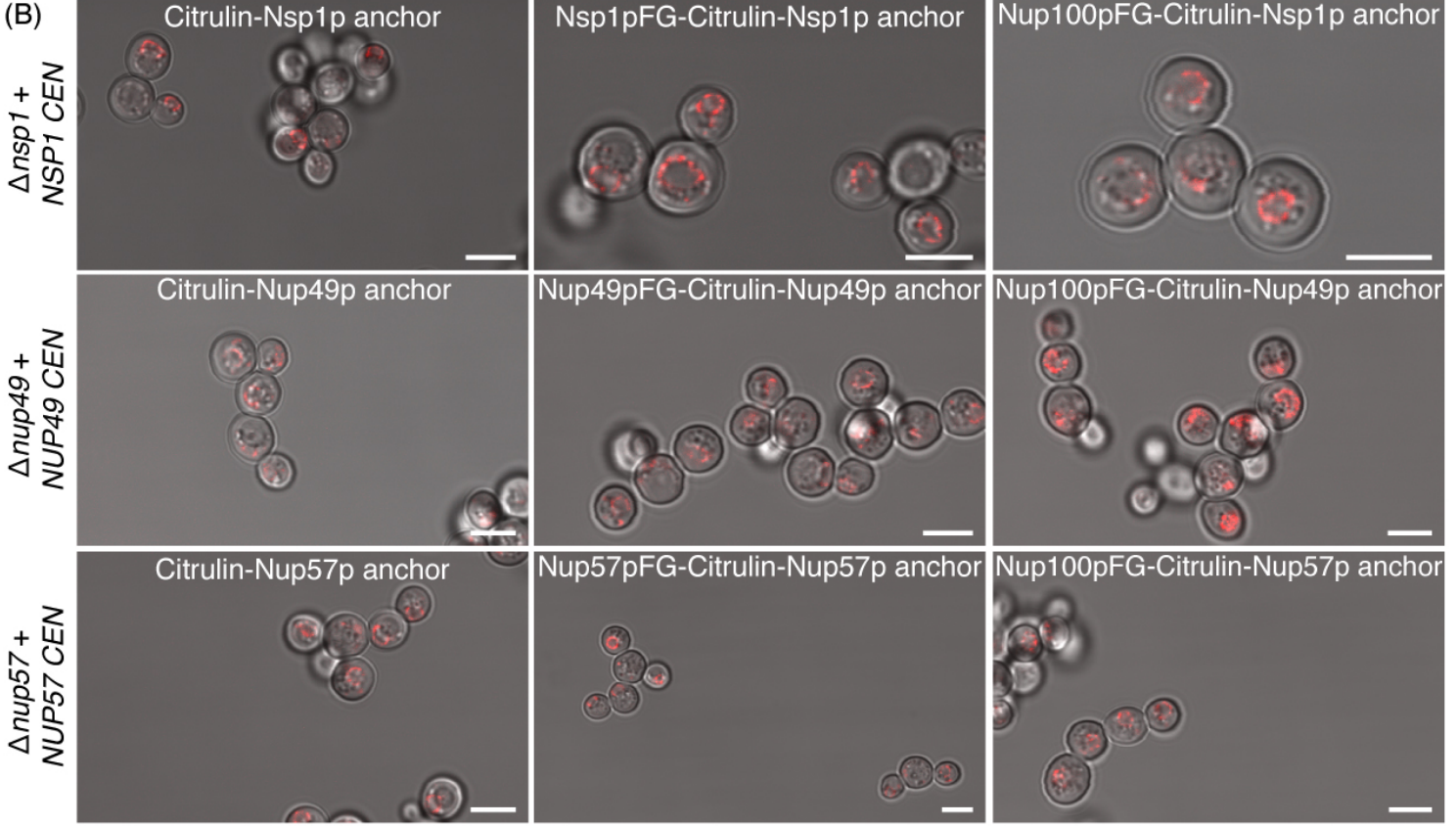

Figure 2.12: Nup100p FG domain fusion proteins incorporate into the NPC: (A) Constructs were transformed into strains with genetic backgrounds as indicated (from top to bottom: YMR29, YMR30, YMR31). The ability of the transformed constructs to complement the loss of an according wild type protein was monitored by an FOA Assay. Cells were incubated for 4 days at $25^{\circ} \mathrm{C}$. The exact amino acid composition of the fusion proteins can be found in section 4.1.10. (B) Cells grown on FOA plates from the assay shown in (A) were analyzed by confocal microscopy as described in section 4.2.3. An overlay of the scattered light image with the fluorescence signal by Citrulin (red) is shown. Scale bars represent $5 \mu \mathrm{m}$. 


\subsubsection{Nup100p FG domain reduces cell growth at various anchor domains}

The Nup100p FG domain fused to various Citrine labeled anchor domains was incorporated into the NPC even though transformed cells showed decreased growth rates. We wondered whether the reduction in growth rate observed was an effect of the Citrine labeling or whether the presence of the Nup100p FG domain interfered with normal growth behavior. Hence, we tested various fusions of the Nup100p FG domain to anchor domains in such genetic backgrounds, where the presence of the endogenous FG domain fused to its respective anchor domain is not essential for cell viability (figure 2.13).

In a $\Delta n s p 1$ strain, the Nsp1p anchor domain is essential, and its sole presence, i.e. without its FG domain, restored cell growth comparable to a wild type situation. In contrast, cells provided with Nup100p $\mathrm{p}^{\mathrm{FG}}-\mathrm{Nsp} 1 \mathrm{p}^{\text {anchor }}$ showed reduced growth compared to the presence of the anchor domain alone.

An analogous phenotype was observed in a $\Delta$ nup 49 strain and in a $\Delta$ nup 57 strain. Clearly, the reduction in growth rate can be attributed to the presence of the Nup100p FG domain. This dominant negative phenotype of the Nup100p FG domain was even more pronounced in a $\Delta$ nup 57 nsp $1 \Delta F G$ nup $49 \Delta F G$ nup $145 \Delta F G$ background. Here, the fusion protein Nup100p ${ }^{\mathrm{FG}}-\mathrm{Nup} 57 \mathrm{p}^{\text {anchor }}$ was not able to rescue viability at all.

On the other hand, in a $\Delta$ nup 57 nsp $1 \Delta F G$ nup $49 \Delta F G$ nup $100 \Delta F G$ background Nup100p ${ }^{\mathrm{FG}_{-}}$ Nup $57 \mathrm{p}^{\text {anchor }}$ rescued viability to a similar extent as the wild type protein did. Notably, in this background the Nup100p FG domain was genomically deleted so that it was only supplied by the transformed fusion protein.

\subsubsection{The negative effect is not caused by exceeding FG mass}

The FG domain of Nup100p is 579 amino acids long ( $58 \mathrm{kD})$. To test whether exceeding FG mass incorporated at the Nsp1p anchor domain was the cause of lethality, we generated chimeric FG domains and anchored them via the Nsp1p anchor domain in the NPC. The chimeric FG domains were Nup57p $\mathrm{p}^{\mathrm{FG}}-\mathrm{Nup} 49 \mathrm{p}^{\mathrm{FG}}$ with a total length of 470 amino acids $(\sim 45 \mathrm{kD})$ and the triple fusion Nup57p $\mathrm{p}^{\mathrm{FG}}-\mathrm{Nsp}_{1} \mathrm{p}^{\mathrm{FG}}-\mathrm{Nup} 49 \mathrm{p}^{\mathrm{FG}}$ with a total length of 1073 amino acids $(\sim 107 \mathrm{kD})$ (figure 2.14 ).

None of these fusions resulted in a dominant negative effect. This argues against the hypotheses that exceeding FG mass fused to the Nsp1p anchor domain negatively impacts viability. 

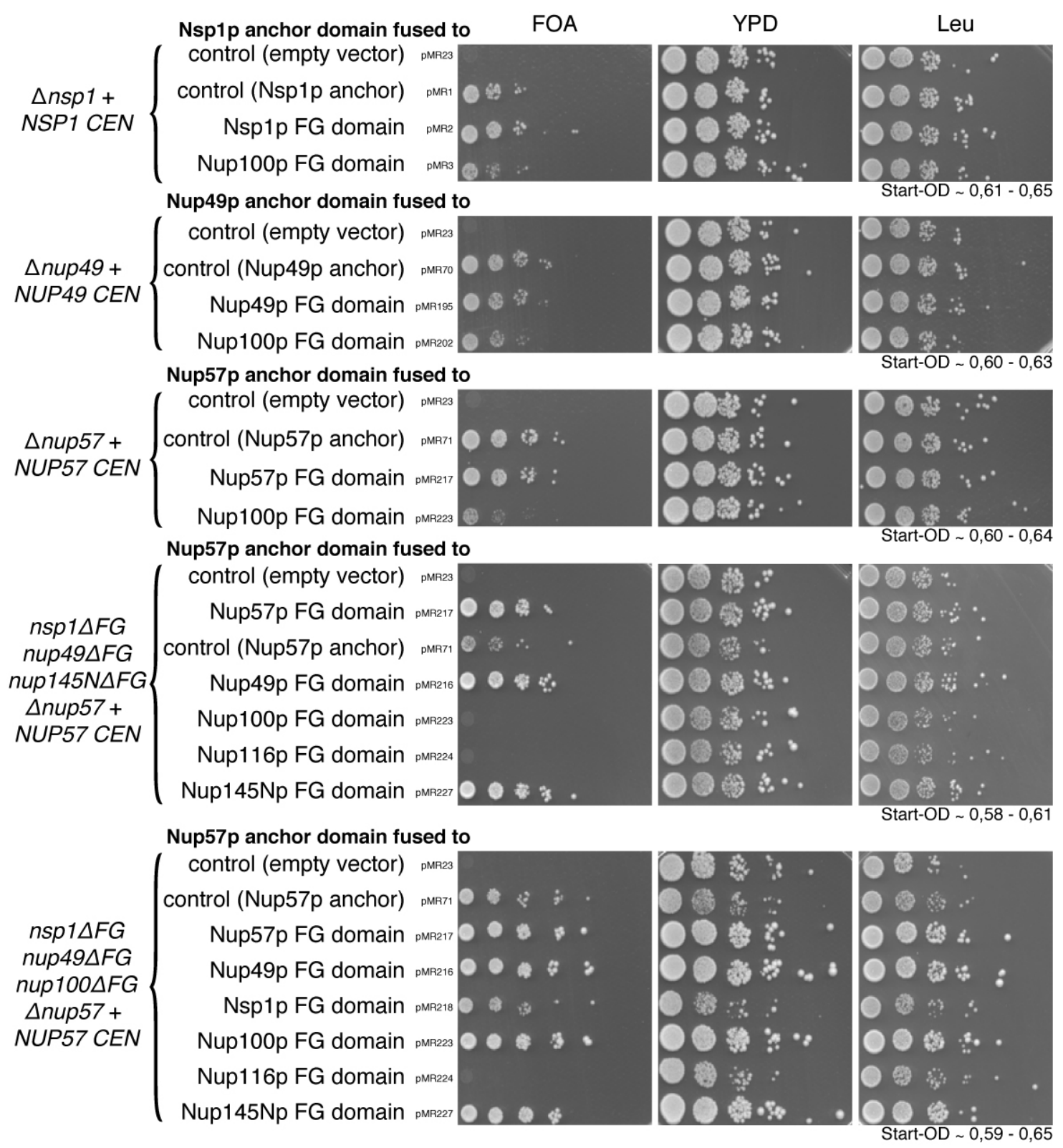

Figure 2.13: Nup100 FG domain fusion proteins show a dominant negative effect: Constructs were transformed into strains with genetic backgrounds as indicated (from top to bottom: YMR29, YMR30, YMR31, YMR78, YMR81). The ability of the transformed constructs to complement the loss of an according wild type protein was monitored by an FOA Assay. Cells were incubated for 3 days (YMR29YMR31), 4 days (YMR81), or 5 days (YMR78) at $25^{\circ} \mathrm{C}$, respectively. The exact amino acid composition of the fusion proteins can be found in section 4.1.10. 


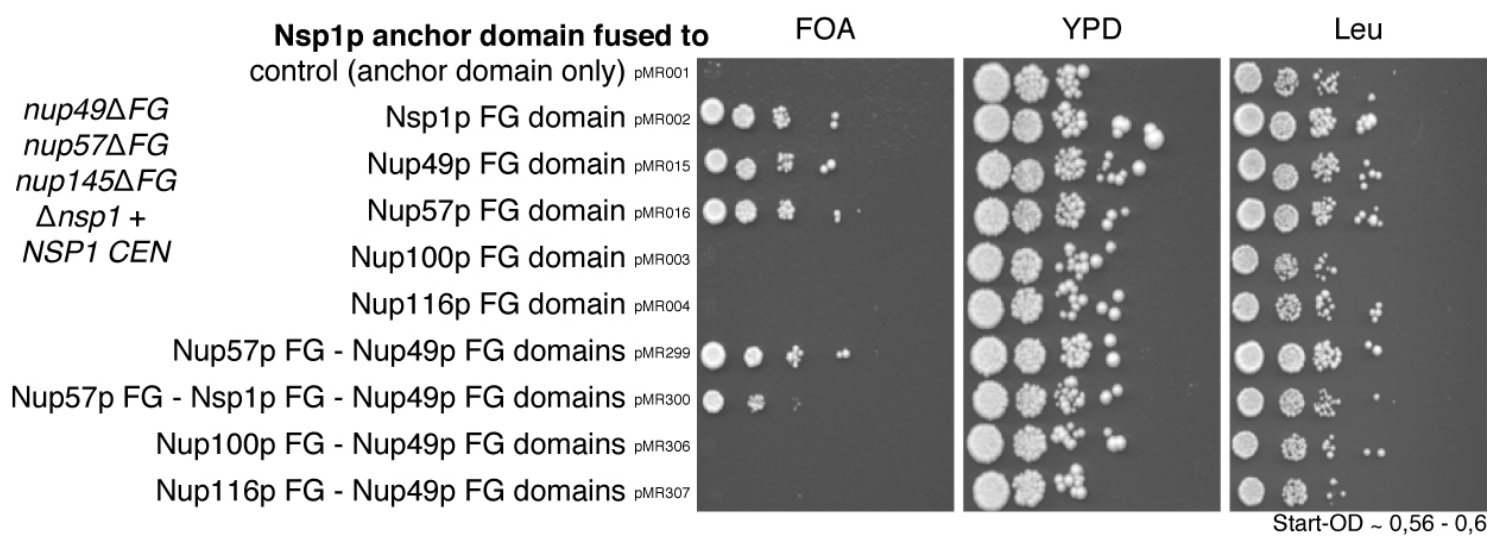

Figure 2.14: Increasing FG mass and junction spacer constructs at the Nsp1p anchor point:

The ability of the transformed constructs to complement the loss of Nsp1p wild type protein was monitored in YMR113 via FOA Assay. Cells were incubated for 6 days at $25^{\circ} \mathrm{C}$. The exact amino acid composition of fusion proteins can be found in section 4.1.10.

\subsubsection{The negative effect is not caused by an inappropriate connection to the anchor domain}

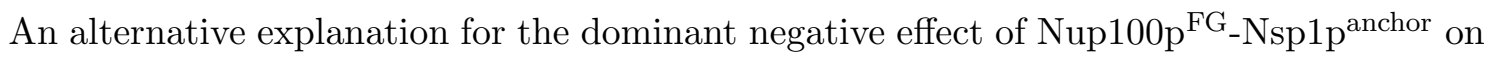
cell growth would be that the artificial connection at the fusion point of the Nup100p- or Nup116p FG domain with the Nsp1p anchor domain is deleterious.

We already knew that Nup49p $\mathrm{p}^{\mathrm{FG}}-\mathrm{Nsp} 1 \mathrm{p}^{\text {anchor }}$ rescues lethality. So we tested the hypotheses by using parts of the Nup49p FG domain as a "spacer" in between the FG domain of Nup100p or Nup116p and the Nsp1p anchor domain. Again, the presence of Nup100p- or Nup116p FG domain caused a lethal phenotype (figure 2.14).

\subsection{FG domain mass is insufficient to predict viability}

One main conclusion by Strawn et al. (2004) was that remaining FG domain mass is insufficient to predict viability or transport efficiency of a given strain. However, we showed that strains lacking multiple FG domains in some combinations, previously reported to be non-viable, remained viable when the FG domains were cleanly deleted.

Thus it is challenging to ask, whether remaining FG mass is a predictive value for viability when re-evaluated with results from our deletion study. We recalculated therefor the remaining FG mass in the NPC for each of the mutant strains. In table 2.1, data are included that result from screening the effect of an additional gene deletion in the multi $\Delta$ FG mutant strains (see section 2.3.2). Like Strawn et al. we were not able to predict 
Table 2.1: Remaining FG mass in multi $\Delta F G$ mutant strains: Strains are sorted according to their remaining FG mass. Remaining FG mass (in $\mathrm{MDa}$ ) was calculated for each mutant strain on the basis of the FG domain boundaries defined in section 2.2 or according to Strawn et al. (2004). The abundance of each FG nucleoporin in the NPC was estimated based on the stoichiometry reported by Rout et al. (2000) and on described FG Nup binding sites in the NPC (8 copies of Nup42p, Nup159p, Nup1p, Nup2p, Nup60p, Nup100p, and Nup116p; 16 copies of Nup49p, Nup57p, and Nup145Np; and 24 copies of Nsp1p). • indicates the presence of the according FG domain in the strain whereas an empty box indicates that the FG domain was deleted.

\begin{tabular}{|c|c|c|c|c|c|c|c|c|c|c|c|c|}
\hline 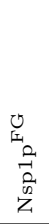 & 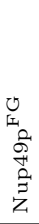 & 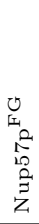 & 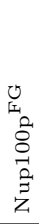 & 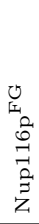 & 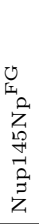 & 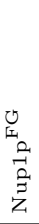 & 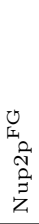 & 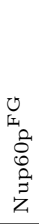 & 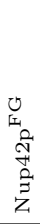 & 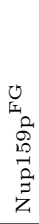 & 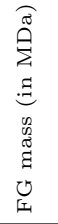 & 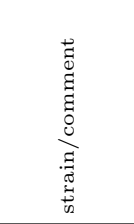 \\
\hline • & $\bullet$ & - & - & - & • & $\bullet$ & - & - & - & - & 4,98 & wild type \\
\hline 0 & - & 0 & 0 & & & - & - & 0 & 0 & 0 & 4,09 & YMR77* \\
\hline 0 & - & 0 & & & 0 & 0 & - & 0 & 0 & 0 & 3,95 & YMR127* \\
\hline 0 & & & 0 & 0 & & 0 & 0 & 0 & 0 & 0 & 3,96 & YMR109 † \\
\hline 0 & - & & - & & & - & - & 0 & 0 & 0 & 3,67 & (1) \\
\hline 0 & & 0 & 0 & & & $\bullet$ & - & 0 & $\bullet$ & 0 & 3,65 & (1) \\
\hline 0 & 0 & 0 & & & & 0 & 0 & 0 & 0 & $\bullet$ & 3,63 & YMR128* \\
\hline 0 & & & - & & - & - & - & 0 & - & 0 & 3,62 & (1) \\
\hline 0 & 0 & & & 0 & & 0 & 0 & 0 & 0 & 0 & 3,61 & (1) \\
\hline 0 & & 0 & & 0 & & 0 & 0 & 0 & 0 & 0 & 3,59 & (1) \\
\hline 0 & & & & 0 & $\bullet$ & 0 & $\bullet$ & 0 & 0 & 0 & 3,57 & (1) \\
\hline 0 & - & 0 & 0 & 0 & - & & & & & & 3,29 & (1) \\
\hline 0 & $\bullet$ & 0 & $\bullet$ & 0 & & & & & & & 2,97 & (2) \\
\hline 0 & - & & - & 0 & - & & & & & & 2,95 & (2) \\
\hline 0 & 0 & 0 & 0 & & 0 & & & & & & 2,89 & $(2)$ \\
\hline & 0 & & 0 & 0 & & 0 & 0 & 0 & 0 & 0 & 2,85 & YMR64 † \\
\hline & & 0 & 0 & 0 & & $\bullet$ & 0 & 0 & $\bullet$ & 0 & 2,83 & YMR120 † \\
\hline 0 & - & 0 & & 0 & - & & & & & & 2,83 & (1) \\
\hline & & & $\bullet$ & 0 & $\bullet$ & 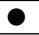 & 0 & 0 & 0 & $\bullet$ & 2,80 & YMR119 † \\
\hline & - & & & 0 & - & - & - & 0 & - & 0 & 2,70 & YMR117 \\
\hline & & 0 & & 0 & 0 & 0 & 0 & 0 & 0 & 0 & 2,68 & YMR118 \\
\hline 0 & 0 & 0 & & 0 & & & & & & & 2,62 & $(2)$ \\
\hline & 0 & 0 & 0 & & & - & - & 0 & - & 0 & 2,59 & (1) \\
\hline & - & & 0 & & - & 0 & - & 0 & 0 & 0 & 2,56 & (1) \\
\hline & & 0 & 0 & & 0 & 0 & - & 0 & 0 & 0 & 2,54 & (1) \\
\hline & - & 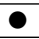 & & 0 & & - & - & 0 & - & 0 & 2,53 & (1) \\
\hline & & & 0 & 0 & & 0 & 0 & 0 & 0 & 0 & 2,49 & YMR68 †† \\
\hline & 0 & 0 & 0 & 0 & $\bullet$ & & & & 0 & 0 & 2,49 & $(1)$ \\
\hline & & 0 & & 0 & & 0 & 0 & 0 & 0 & 0 & 2,37 & YMR122 † \\
\hline & & & & 0 & - & 0 & - & 0 & 0 & 0 & 2,34 & YMR123 † \\
\hline & - & 0 & 0 & 0 & 0 & & & & & & 1,87 & $(1)$ \\
\hline & & & & & 분 & - & - & - & 0 & 0 & 1,77 & YMR124 \\
\hline
\end{tabular}

- FG domain present / not deleted

* strain with transplanted GLEBS domain, see section 2.3.1.1

$\dagger$ strain non-viable when generated by recombinase strategy, but viable in this study $\ddagger$ viability assayed by FOA assay

(1) strain generated by Strawn et al. (2004), genotype not reproduced in this study

(2) strain generated by Terry and Wente (2007), genotype not reproduced in this study viable strain

non-viable strain 
viability of cells based on remaining FG mass.

However, it was argued that avidity effects due to cohesion mediated by the FG domains contribute to the incorporation of FG Nups into the NPC (Griffis et al., 2003, Hülsmann et al., submitted). Therefore it might well be that an FG domain deleted Nup is not able to bind to its endogenous binding site within the NPC any longer and that this free binding site is consequently occupied by an other FG Nup. For example, it was shown that Nup100p and Nup145Np can attach to the binding site of Nup116p in vitro (Yoshida et al., 2011). Such possible replacements were not considered for the calculation of remaining FG mass. 


\section{Discussion}

Nuclear pore complexes (NPCs) provide the transport route for nucleocytoplasmic exchange (Feldherr, 1962). They establish the permeability barrier (Paine et al., 1975) that excludes inert macromolecules (Paine and Feldherr, 1972; Mohr et al., 2009) but permits facilitated passage of even very large cargoes, provided these are bound to nuclear transport receptors (NTRs, reviewed e.g. by Görlich and Kutay, 1999).

There is strong evidence that FG domains are the building blocks of the permeability barrier:

(i) NTRs bind to FG motifs (e.g. Iovine et al., 1995; Radu et al., 1995; Bayliss et al., 2000, 2002a,b) and this binding was found to be essential for facilitated NPC passage (Bayliss et al., 1999). Even more, this binding accelerates NPC passage of the NTR·cargo complex by a factor of 100-1000 compared to the passage of an inert objects of similar size (Ribbeck and Görlich, 2001).

(ii) FG domains radiate into the central channel of the NPC (Akey and Goldfarb, 1989; Grote et al., 1995). It has been estimated that more than 1000 copies of FG repeats are within one NPC, and that their local concentration in the interior of the central channel approximates $50 \mathrm{mM}$ (Bayliss et al., 1999), which would be sufficient to establish a functional barrier (Ribbeck and Görlich, 2001; Frey and Görlich, 2007).

(iii) FG domains spontaneously form macroscopic hydrogels when a critical protein concentration is exceeded (Frey et al., 2006), and these macroscopic hydrogels reproduce permeability barrier properties of authentic NPCs (Frey and Görlich, 2007, 2009; Milles and Lemke, 2011).

(iv) With the onset of Aspergillus nidulans closed mitosis, all FG Nups disperse throughout the cell, whereas the majority of the structural Nups remain at the NPC (Souza et al., 2004; Osmani et al., 2006). Concomitant, proteins that exclusively locate either to the nucleoplasm or the cytoplasm during interphase equilibrate between the two compartments (Suelmann et al., 1997; Ovechkina et al., 2003). Likewise, phosphorylation of vertebrate 
Nup98 was suggested to be crucial for mitotic disassembly of the permeability barrier and inhibition of phosphorylation retards NE permeabilization (Laurell et al., 2011).

(v) Upon infection with poliovirus, nuclear proteins leak into the cytoplasm (Belov et al., 2004) and the active import of proteins is suppressed (Gustin and Sarnow, 2001). Associated with this perturbation is the observation that several FG Nups are released from the NPC by the activity of viral proteases (Gustin and Sarnow, 2001, 2002; Park et al., 2008).

\subsection{Experimental systems for the analysis of the permeability barrier}

Even though the role of FG domains as main building blocks of the permeability barrier is well established, the question how FG domains achieve selectivity while allowing very fast translocation of transport complexes is a central issue in the field. However, addressing this question is challenging since it is not obvious which features of FG domains are important for permeability barrier functionality in vivo.

\subsubsection{Differences among FG domains}

The $S$. cerevisiae permeability barrier is constituted of FG domains originating from 11 distinct nucleoporins (Rout et al., 2000). Thus, heterogeneity is a prominent feature in vivo. Besides the fact that several Nups with distinct binding sites within the NPC contribute FG domains, FG domains themselves are also very heterogeneous since they can differ in a multitude of features: (i) their exact FG motif, with "FG", "GLFG", "FxFG", or "FxF" being the prominent ones in yeast, (ii) their length, ranging from around 100 amino acids for the Nup60p FG domain to more than 700 amino acids for the FG domain of Nup116p, (iii) the sequence of their inter-repeat spacer, or (iv) the distribution and frequency of individual amino acids. For example, even though most of the FG domains do not exhibit an overall net charge, they differ in the relative number of charged amino acids $^{1}$. It was proposed that the ratio of charged to hydrophobic amino acids relates to different proposed categories of intrinsically disordered structures for FG domains (Yamada et al., 2010).

However, it is not known whether any of this diverging features is of functional significance

\footnotetext{
${ }^{1}$ More than $20 \%$ of the amino acids from the FG domains of Nsp1p, Nup1p, Nup2p, Nup60p, and Nup159p comprise charged amino acids (Asp, Glu, Lys, Arg) whereas the other yeast FG domains exhibit less than $5 \%$ charged amino acids.
} 
or whether heterogeneity as such is an essential feature for in vivo NPC function.

We know that binding of NTRs to FG motifs is not only essential for facilitated NPC passage (Bayliss et al., 1999) but also accelerates NPC passage of an NTR-cargo complex (Ribbeck and Görlich, 2001). Therefore, NTR binding sites are a crucial functional feature of FG domains.

The hydrophobic clusters of FG domains not only bind NTRs but they also create interrepeat contacts (Frey et al., 2006; Patel et al., 2007; Frey and Görlich, 2009; Ader et al., 2010; Yamada et al., 2010). Intermolecular interaction between FG domains is determined by their overall cohesiveness. Additionally, it was observed that FG domains bind to nonFG domains of Nups in vitro (Patel et al., 2007; Schrader et al., 2008).

In addition to the listed characteristics, there may be more yet unknown but functionally important features.

\subsubsection{Limits of current experimental systems}

So far several types of experimental setups analyzed aspects of FG domain features for permeability barrier functionality.

Recombinantly expressed FG domains were found to form macroscopic hydrogels (Frey et al., 2006). These FG hydrogels display selectivity properties very similar to authentic nuclear pores (Frey and Görlich, 2007, 2009). Using FG hydrogels as a model system allows to study facilitated and passive transport in vitro. The system was e.g. used to quantify and compare partition coefficients and entry rates of different molecules in hydrogels derived from distinct FG domains (Frey and Görlich, 2009). Additionally it was shown that hydrogel formation not only depends on hydrophobic interactions but as well on hydrophilic contacts (Ader et al., 2010) specifying further the molecular basis of FG domain cohesiveness. Thus, hydrogels can be a very powerful tool to evaluate the effect of FG domain mutations on transport processes.

However, so far hydrogels were only formed by one kind of FG domain or by a chimeric fusion protein of up to three FG domains, therewith limiting the system for the analysis of heterogeneity. Even more, it is unclear whether hydrogel formation occurs in authentic NPCs and whether it is required for in vivo NPC function.

The significance of heterogeneity was addressed by genetic studies in yeast deleting FG domains in vivo (Fabre et al., 1995; Strawn et al., 2004; Terry and Wente, 2007). These 
studies showed that FG domains build up the permeability barrier in a highly redundant manner, and further indicated that FG domains are not equivalent to each other. Thus, a certain degree of heterogeneity seems to be essential for NPC functionality in vivo. However, previous studies did not further specify the need for heterogeneity, neither they addressed the question whether the anchoring points of FG domains within the NPC are of functional significance.

Artificial nanopores that were thought to resemble certain characteristics of NPCs were used to observe transport events or behavior of an ensemble of FG domains (JovanovicTalisman et al., 2009; Kowalczyk et al., 2011). In theory, such a system would combine the possibility to manipulate FG domains - and thus to test the effect of changing FG domain features - with the potential to create a "pore" that consists of several distinct FG domains. In hitherto presented systems, FG domains were however immobilized on surfaces and it was assumed that they radiate into the generated channel. This is problematic for two reasons: First, such a model does not accurately mimic the situation in the NPC, where FG domains are grafted inside the translocation channel. Second, the FG domain covered surface provides a multitude of additional binding sites for NTR $\cdot$ cargo complexes. Even more, the systems presented so far did not accomplish selectivity that is in the range of selectivity provided by authentic NPCs.

Computational studies simulating NPCs tried to "observe" transport events in silico (e.g. Miao and Schulten, 2009; Mincer and Simon, 2011; Moussavi-Baygi et al., 2011). These studies can be grouped into two categories. All atom molecular dynamics simulations (Miao and Schulten, 2009) allow to accurately study interactions between NPC components but are limited to a very short time scale of nanoseconds to microseconds. However, transport events occur in the range of several milliseconds (Kubitscheck et al., 2005; Yang and Musser, 2006). In contrast, other simulations model longer time scales but to do so they have to reduce the complexity of the system by making prior assumptions ${ }^{2}$. For example, FG domains are modeled as inert filaments interacting solely via FG motifs ${ }^{3}$

\footnotetext{
${ }^{2}$ For a simulation of transport reactions it would be necessary to calculate interactions of more than 800000 atoms for the FG mass plus the mass contributed by multiple NTR·cargo complexes. This is not feasible since computing power is still a limiting factor

${ }^{3}$ Hitherto, binding of FG motifs was simulated with one defined FG-FG off rate. Doing so does not account for the fact that NTR binding to FG motifs increases the off rate.
} 
neglecting interactions mediated by the spacer sequences (Mincer and Simon, 2011).

In summary, all these studies are limited or biased to a certain extent. To clarify the nature of the permeability barrier without a prior bias, the significance of distinct FG domain features and predictions from transport models have to be tested in the context of real NPCs. This might be done by analyzing living cells, native NPCs in permeabilized cells, or NPCs in an engineered system.

To test hypotheses about the significance of FG domain features it is not only necessary to delete individual FG domains but also to integrate artificial FG domains with modified domain features into the NPC. Therefore, a more modular and flexible experimental system is needed.

Experiments performed in such a modular system should allow to clearly evaluate the significance of distinct FG domain features for the functionality of the permeability barrier in vivo.

\subsubsection{New experimental systems to study the NPC permeability barrier}

The development of such a system was very recently reported by Hülsmann et al. (manuscript submitted). This system is based on in vitro assembled nuclei from Xenopus laevis egg extract and allows the incorporation of artificial FG domains into authentic NPCs. Selectivity and tightness are then tested by simultaneously challenging the modified permeability barrier with inert molecules and NTR·cargo complexes.

Here, we report the development of a complementary system based on genetically modified Saccharomyces cerevisiae strains. We employed this in vivo yeast system to evaluate FG domain features for barrier functionality and to test central predictions of the proposed permeability barrier models.

Like the system by Hülsmann et al., the in vivo yeast system allows to incorporate artificial FG domains at various anchor points in the NPC. Functionality of the permeability barrier is assayed by testing cell viability.

In our system, living cells are indicative for the proper function of all physiologically essential nuclear import and export pathways. Living cells allow to additionally test the consequences of cell stress on barrier function. More over, the yeast based system does not rely on recombinantly expressed proteins. Thus, it allows to analyze synthetic FG 
domains that cannot be expressed or purified for usage in the Xenopus system.

However, the in vivo yeast system has one disadvantage compared to the X. laevis egg extract system. So far, we cannot distinguish whether a resulting lethal phenotype of an integrated FG domain is caused by (i) a non-selective barrier that results in uncontrolled mixing of cytoplasmic and nuclear content, (ii) a barrier that is too tight so that any transport is prevented, (iii) by the blockade of one specific transport pathway.

In summary, both systems can be used to assay the consequences of fusing any FG domain to one specific anchor point for barrier functionality in authentic NPCs.

\subsubsection{Establishment of the yeast genetic system}

Our strategy to develop an in vivo yeast system relied on the use of previously described FG domain deletion strains (Strawn et al., 2004). We needed a strain with a lethal combination of deleted FG domains. Viability in such a strain should only be re-established by the presence of one specific FG domain. By genetically replacing this FG domain with modified FG domains and testing the consequences for viability we wanted to extrapolate critical features of FG domains for viability.

For the generation of their FG domain deletion strains, Strawn et al. (2004) applied a strategy that eventually resulted in an N-terminal extension of the FG domain-deleted nucleoporin. These additional amino acids (the "scar domain" of the deletion strategy) are coded by a remaining recombinase recognition site. We showed here that this scar is causative for a cumulatively deleterious effect in multi $\Delta \mathrm{FG}$ mutant strains. Furthermore, we demonstrated that the addition of a single scar domain could convert a normally viable multi $\Delta$ FG mutant strain into a non-viable strain.

For this reason, the previously generated multi $\Delta \mathrm{FG}$ mutant strains were not suitable as starting point for our genetic assay. If we had used them, we would not have been able to differentiate between a phenotype caused by the scar domain and a phenotype related to a modification within the FG domain of interest.

We thus had to start generating "clean" multi $\Delta \mathrm{FG}$ mutant strains.

\subsubsection{A new FG domain deletion strategy was needed}

The generation of "clean" deletion strains useful for the above mentioned assay was faced with two general challenges. 
(i) The yeast $S$. cerevisiae harbors 11 FG Nups. To analyse all possible combinations of FG domain deletions, we would have needed to generate 2047 strains $^{4}$. Generating this number of strains seemed unfeasible.

However, Strawn et al. (2004) reported that the deletion of all peripherally localized FG domains, namely Nup1p, Nup2p, Nup42p, Nup60p, and Nup159p, results in a viable strain. Furthermore, these FG domains were proposed to primarily function in the disassembly of import or export complexes (Wälde and Kehlenbach, 2010). We therefore assumed that these FG domains do not considerably participate in establishing the peremability barrier. For the generation of a starting strain for our intended assay, we thus focused on deleting only the centrally localized FG domains, namely Nsp1p, Nup49p, Nup57p, Nup100p, Nup116p, and Nup145Np. This reduced the number of possible combinations to 64. Even more, Strawn et al. (2004) reported that nearly all $\Delta$ FG mutant strains deficient in only one or two FG domains were viable. Thus we did not need to generate all possible double FG domain deletions ${ }^{5}$, reducing the number of strains to be generated to 43 at most.

(ii) Two general strategies seem to allow the efficient generation of strains lacking multiple FG domains in various combinations. The strains can be generated either by using a recombinase system or by applying an elaborated scheme of combining $\Delta \mathrm{FG}$ mutant alleles via mating and sporulation. However, in this study we showed that the use of a recombinase based strategy previously resulted in a cumulatively lethal effect. On the other hand, deficiency in some nucleoporins was shown to result in cell division phenotypes (Baï et al., 2004). Thus a strategy based on mating and sporulation seemed not feasible either.

We decided to employ a strategy based on yeast integration plasmids in combination with selection marker recovery via "loop-out" (for details see section 4.2.2.4). This strategy allows to successively delete any number of FG domains without being limited by the number of available selection marker.

\subsubsection{FG domains operate in a redundant manner}

Pursuing this strategy, we generated a set of new multi $\Delta \mathrm{FG}$ domain deletion strains that have "clean" genomic deletions, i.e. just the FG domain coding sequence is removed and

\footnotetext{
${ }^{4}$ Any FG domain can either be present or deleted. These two possibilities apply to all of the 11 FG Nups, resulting in $2^{11}$ possible combinations. However, we do not need to generate the wild type strain, resulting in $2^{11}-1=2047$ combinations.

${ }^{5}$ The number of possible combinations is calculated by the binomial coefficient $\left(\begin{array}{l}n \\ k\end{array}\right)$ with n being the total number of considered FG domains and $\mathrm{k}$ being the number of combined deletions. There are $\left(\begin{array}{l}6 \\ 1\end{array}\right)=6$ possible single deletions and $\left(\begin{array}{l}6 \\ 2\end{array}\right)=15$ combinations for double deletions.
} 
no additional sequences are inserted.

In contrast to previous reports (Strawn et al., 2004), four triple combinations of FG domain deletions resulted in viable strains when the FG domains were "cleanly" deleted. Additionally we reported the viability of three quadruple $\Delta F G$ domain strains (see table 2.1 on page 41$)$.

Thus, we enlarged the number of viable multi FG domain deletion strains and therewith corroborated the previous statement that FG domains function in a highly redundant manner (Fabre et al., 1995; Strawn et al., 2004). We conclude that remaining FG mass is insufficient to predict viability or transport efficiency of a given strain. Therefore, FG mass per se seems not to be an essential criterium for permeability barrier functionality.

We next assayed the effects of additional complete gene deletions in the multi $\Delta \mathrm{FG}$ mutant strains. If viability of a strain depends on the presence of the FG domain coded by the previously deleted gene, we can use this strain to assay the effect on viability when this FG domain is replaced by other domains. By doing so, we identified three strains, which fulfill this criterion and could thus be used for the in vivo yeast assay.

\subsubsection{FG domains of the Nsp1 subcomplex are not essential}

We show that all FG domains of the Nsp1 subcomplex, i.e. Nsp1p, Nup49p, and Nup57p, can be deleted simultaneously without affecting cell viability.

Fabre et al. (1995) already concluded the viability of such a triple FG domain deletion strain whereas Strawn et al. (2004) concluded the opposite.

However, there were several differences between these studies: First, Fabre et al. only partially deleted the FG domain from Nsp1p. Additionally, the first 12 amino acids of Nup49p were not deleted. Second, Strawn et al. performed chromosomal deletions of the FG domains with all mutant alleles being under the control of their endogenous promoters. Fabre et al. expressed FG domain deficient Nup49p and Nup57p from a plasmid, with nup $57 \triangle F G$ being under the control of the NOP1 promoter. And third, the studies differ in the genetic background of the used yeast strains. It was therefore argued that the discrepancy in the observed phenotypes could be due to a difference in the deleted sequences, protein expression levels, or yeast strain background (Strawn et al., 2004).

It is known that NUP1 becomes non essential in the presence of a suppressor mutant background (Belanger et al., 1994). Thus, the background of a deletion might strongly 
influence its phenotype.

We chose the same genetic background as Strawn et al. and deleted nearly the same sequences. Only the deleted sequence for Nsp1p slightly varies ( $\Delta 3-602$ in this study versus $\Delta 13-591$ in Strawn et al., 2004). We therefore expected to reproduce the phenotype previously described by Strawn et al. (2004).

However, we could show that the lethal phenotype of a nsp $1 \Delta F G$ nup $49 \Delta F G$ nup $57 \Delta F G$ strain observed by Strawn et al. is only caused by a deleterious effect of the "scar domain". If generated with "clean" FG domain deletions, this combination of deletions results in a viable strain.

This result is in line with the previous observation that the NPC-binding site for Nsp1p on Nic96p is not required for viability, even though deletion of this binding site results in a severe decrease in growth rate (Schrader et al., 2008).

We have not evaluated the mechanism of how the additional amino acids of the "scar domain" negatively impact cell viability further.

It is, however, tempting to speculate about the mechanism. More than one third of the amino acids of the "scar domains" are hydrophobic. It is well possible that scar domains somehow interact with parts of the FG domains or with themselves. Thereby they could form a conglutination that clogs the transport channel of the NPC. This would also explain why a single FG domain deletion via recombinase-driven excision has no detectable effect but the combination of such deletions results in cell death.

\subsubsection{Re-evaluation of former results is needed}

We showed that more FG domains can be simultaneously deleted without affecting viability than previously reported (Strawn et al., 2004; Terry and Wente, 2007). We could attribute the partially drastic differences in reported phenotypes to the presence of a "scar domain" in previously generated yeast strains.

As a consequence, it seems reasonable to re-evaluate conclusions that were drawn by making use of $\Delta \mathrm{FG}$ mutant strains created by Strawn et al. (2004) ( $\Delta \mathrm{FG}^{\text {scar }}$ strains).

For a re-evaluation, three kinds of conclusions need to be distinguished as exemplified in the following: (i) Strawn et al. (2004) concluded that FG domains function in a highly redundant manner as a third of the yeast FG mass can be deleted without affecting cell viability. This conclusion, even though obtained by $\Delta \mathrm{FG}^{\text {scar }}$ strains, holds true since strains should be viable when the same combinations of FG domains are cleanly deleted as compared to viable $\Delta \mathrm{FG}^{\text {scar }}$ strains. 
(ii) By comparing which FG domains are present in viable $\Delta \mathrm{FG}^{\text {scar }}$ and non-viable $\Delta \mathrm{FG}^{\text {scar }}$ strains, Strawn et al. extrapolate requirements for minimal functional NPCs. This kind of conclusion needs to be reevaluated since some $\Delta \mathrm{FG}^{\text {scar }}$ strains are viable when cleanly generated (see table 2.1 on page 41 ).

(iii) Transport of NTR $\cdot$ cargo complexes or reporter constructs was studied to reveal defects in specific transport pathways (Strawn et al., 2004; Terry and Wente, 2007; Meinema et al., 2011). An observed decrease in transport rates was attributed to the absence of a specific subset of FG domains. However, it might as well be attributed to the presence of the scar domain that might strongly bind NTR-cargo complexes or reporter constructs. Thereby the complexes would be retained in the NPC. In this case, the experiments should be repeated in clean multi $\Delta \mathrm{FG}$ strains to evaluate whether the presence of additional amino acids impacts the results.

\subsection{Role of FG domain anchor points}

We identified three strains, in which a lethal combination of deleted FG domains was only rescued by the presence of one specific FG domain. These strains allowed to test the effect of fusing any FG domains to the anchor domains of Nup100p, Nup116p, and Nsp1p on viability.

We first employed these strains to test whether endogenous FG domains can functionally substitute each other. In summary, we observed that endogenous FG domains can be grouped into three classes:

(i) The FG domains of Nup60p and Nup159p do not rescue cell viability at any of the tested anchor domains. With only four FG motifs within its 116 amino acids, the Nup60p FG domain might be too short or too low in FG motifs density to functionally replace any other FG domain. But these arguments do not apply to the FG domain of Nup159p. However, the FG domains of Nup60p and Nup159p are the only yeast FG domains with a negative net charge $^{6}$ (for amino acid composition analysis see e.g. Patel et al., 2007). Thus the negative net charge might be the cause for the inability of Nup60p $\mathrm{p}^{\mathrm{FG}}$ and Nup159p $\mathrm{p}^{\mathrm{FG}}$ to functionally replace other FG domains. To test this assumption, we could generate FG domain mutants, in which Asp and Glu are exchanged to Lys and Arg and vice versa and test their effect on viability when fused to various anchor domains. We further could analyze mutants, in which Asp and Glu are mutated to non-charged amino acids.

\footnotetext{
${ }^{6}$ Nup60p FG domain: 5 Asp, 9 Glu, 10 Lys, 2 Arg; Nup159p FG domain 5 Asp, 6 Glu, 7 Lys, 0 Arg
} 
(ii) FG domains from Nup100p and Nup116p only result in viable cells when fused to the anchor domains of Nup100p or Nup116p.

(iii) All other endogenous FG domains can functionally replace the Nsp1p FG domain.

Both of the two last-mentioned classes comprise FG domains that were shown to provide nuclear transport receptor (NTR) binding sites (Iovine et al., 1995; Bayliss et al., 1999, 2000, 2002a,b; Bednenko et al., 2003; Isgro and Schulten, 2005). Nevertheless, fused FG domains are either functional at one or the other anchor domain. This indicates that the ability of an FG domain to bind NTRs is not sufficient to functionally replace any other FG domain.

Furthermore, we show with these result for the first time that FG domain anchor points are not equivalent. Thus, FG domain function not only depends on properties of the FG domain itself but additionally on its anchor point within the NPC.

\subsection{Role of FG domain cohesion for in vivo permeability barrier functionality}

The "selective phase" model assumes that permeability barrier-forming FG domains mediate multivalent intra- and inter molecular interactions via their cohesive parts. Thereby they create a sieve-like meshwork that prevents diffusion of molecules with a diameter above a size limit defined by the mesh size. NTR cargo complexes bind to FG motifs and as a consequence disengage FG meshes. This allows their passage through the meshwork (Ribbeck and Görlich, 2002; Frey and Görlich, 2007).

According to the "selective phase" model, cohesiveness and the ability to bind NTRs are thus central features of FG domains.

Previous experiments demonstrated that recombinantly expressed and purified cohesive FG domains indeed form a macroscopic hydrogel that reproduces main properties expected for authentic NPCs (Frey and Görlich, 2007, 2009).

\subsubsection{Cohesive FG domains are essential for in vivo permeability barrier function}

Very recently it was shown that the Nup98 FG domain is the main constituent of the vertebrate's permeability barrier (Hülsmann et al., manuscript submitted). Moreover, the highly cohesive nature of the Nup98 FG domain was shown to be the crucial determinant for a functional barrier and Nup98 FG domain-deficient NPCs failed in establishing an 
efficient barrier. This phenotype could only be rescued by re-addition of the Nup98 FG domain or by presence of other cohesive FG repeats (Hülsmann et al., manuscript submitted).

We observed that most triple combinations of FG domain deletions resulted in viable strains. However, in line with the results by Hülsmann et al., already the deletion of the FG domains from two yeast homologs of Nup98, Nup100p and Nup116p resulted in non-viable cells, as reported previously (Iovine and Wente, 1997).

The FG domain of Nup116p harbors the so-called GLEBS domain and it was shown that a strain with a nup116 $G L E B S$ mutant allele exhibits clusters of herniated NPCs and is temperature sensitive (Bailer et al., 1998). However, in contrast to Iovine and Wente (1997) we can exclude that the lethal phenotype of the double deletion (nup100 $\mathrm{FG}$ nup $116 \Delta F G)$ is partially caused by the deletion of the GLEBS domain as we functionally transplanted it to Nsp1p prior to the deletion of the Nup116p FG domain. This suggests a prominent functional role of the FG domains of Nup100p and Nup116p for cell viability.

By shortening of the Nup100p FG domain, we learned that a critical FG mass at the Nup100p anchor domain is needed for cell viability when assayed in a nup $116 \Delta F G$ background. However, FG mass fused to the Nup100p anchor alone is not sufficient to constitute a functional permeability barrier (see section 2.3.1.2).

The FG domains of Nup100p and Nup116p are very cohesive, probably even more cohesive than the FG domain of Nup98 (K. Kirli, personal communication). Analogous to the vertebrate NPC, we therefore assume that the FG domains of Nup100p and Nup116p are main constituents of the yeast permeability barrier.

The FG domains of Nup100p and Nup116p cannot be functionally be replaced by any other endogenous FG domain. Nevertheless, an artificially elongated version of the Nup145Np FG domain was able to functionally replace them partially. We predict that also other endogenous cohesive FG domains could functionally replace the FG domains of Nup100p or Nup116p in vivo when artificially elongated.

For example, the N-terminal FG subdomain of Nsp1p (Nsp1p $\left.{ }^{2-175}\right)$ was found to be very cohesive (Ader et al., 2010; Yamada et al., 2010) and preliminary data indeed indicated that this FG subdomain can functionally replace the Nup116p FG domain when a quadrupled mutant of the Nsp1p ${ }^{2-175}$ FG subdomain is fused to the Nup116p anchor domain. This experiments remains to be repeated and the results carefully analyzed. 


\subsubsection{Can the NPC channel be blocked by an excessive presence of cohesive FG mass?}

We observed that the FG domains of Nup100p and Nup116p inhibit cell growth when fused to the anchor domain of nucleoporins that are part of the Nsp1 subcomplex, i.e. Nsp1p, Nup49p or Nup57p. However, this growth inhibition only becomes apparent in strains where Nup100p $\mathrm{p}^{\mathrm{FG}}$ and Nup116 $\mathrm{p}^{\mathrm{FG}}$ are present at their endogenous anchor points. In contrast, cell growth with a Nup100 $\mathrm{p}^{\mathrm{FG}}-\mathrm{Nup} 57 \mathrm{p}^{\text {anchor }}$ construct was normal when analyzed in a nup100 $\triangle F G$ background (see figure 2.13, lowest panel, page 39 ).

One explanation for this phenomenon is that the fusion of FG domains from Nup100p or Nup116p to anchor domains of Nsp1 subcomplex proteins created a permeability barrier that simply was too tight to allow nuclear transport. We suggest that an excessive presence of these cohesive FG domains in the central part of the NPC clogs the pores in a way that facilitated transport is delayed or restricted.

To test this assumption, we will generate two sets of Nup100p FG domain mutants and assay whether they can functionally replace the FG domain of Nsp1p. In one set, the Nup100p FG domain will be gradually shortened. Thus, the incorporated cohesive FG mass will be reduced. For the second set of mutations, we want to reduce the cohesiveness of the Nup100p FG domain e.g. by exchanging hydrophobic amino acids to nonhydrophobic amino acids.

We expect that at least some of these mutants can functionally replace the FG domain of Nsp1p so that cell growth is not inhibited.

\subsection{Role of FG domain heterogeneity}

In this study, we reason that the FG domains of Nup100p and Nup116p are main constituents of the yeast permeability barrier. But what is the function of the nine other endogenous FG domains if mainly Nup100p and Nup116p FG domains build up the permeability barrier?

It was previously proposed that FG domains from the peripheral Nups might primarily function in the disassembly of import or export complexes (reviewed by Wälde and Kehlenbach, 2010). Even though the peripheral FG Nups might therefore not be involved in maintaining the permeability barrier, yeast features six distinct FG Nups that are thought to localize to the central channel of the NPC and thus are thought to have a primary role in formation of the permeability barrier (Terry and Wente, 2009). 


\subsubsection{Do less cohesive FG domains regulate the tightness of the yeast permeability barrier?}

The Nup $100^{\mathrm{FG}_{-} \mathrm{Nsp}} 1^{\text {anchor }}$ construct provoked only a slight delay of growth when assayed in a $\Delta n s p 1$ background. (Please note that in this genetic background all other endogenous FG domains were unaffected and thus present in the NPC.) However, the same construct caused a much more severe phenotype, i.e. non-viability, when assayed in a background where several FG domains were genomically deleted ( $\Delta$ nsp 1 nup $49 \Delta F G$ nup $57 \Delta F G$ nup $145 \Delta F G$ ). If it is the addition of $\mathrm{FG}$ mass that caused this effect, we would however expect exactly the opposite behavior, since addition of cohesive FG mass would more likely result in a blocked NPC channel when the channel is already completely equipped with the endogenous FG domains.

We can overcome this conflict by the following assumption. The permeability barrier of yeast is mainly constituted by the cohesive FG domains of Nup100p and Nup116p. But such a permeability barrier is too tight to allow the transport of very large NTR $\cdot$ cargo complexes due to the strong cohesive properties of these domains. To modulate the barrier, less cohesive or non-cohesive FG domains could "dilute" the barrier.

In an FG domain deletion background the supplemental added FG domain of Nup100p cannot be sufficiently blended by the other remaining FG domains, resulting in the lethal phenotype.

A similar mechanism of "permeability blending" was previously observed in macroscopic hydrogels formed by the FG domain of Nsp1p. A hydrogel formed by the very cohesive N-terminal FG subdomain was so tight that NTR - cargo complexes only accumulated at the surface of the gel and diffusion into the gel was blocked. The regular "FSFG" subdomain alone did not form a hydrogel (Ader et al., 2010). In contrast, both domains together formed a hydrogel that restricted diffusion of inert molecules but highly enriched NTR · cargo complexes thus resembling permeability properties of authentic NPCs (Frey and Görlich, 2007; Ader et al., 2010).

\subsubsection{Vertebrates regulate the tightness of their permeability barrier}

The hypothesis that yeast NPCs regulate the tightness of their permeability barrier by "blending" too cohesive FG domains with less cohesive FG domains is well in line with other findings. The vertebrate homolog of yeast Nup100p and Nup116p is Nup98. Its FG 
domain was found to be highly cohesive and it can form an extremely dense macroscopic hydrogel which is restrictive towards inert molecules. However, this in vitro hydrogel is so dense that even NTR-cargo complexes are not allowed to efficiently enter the gel (A. Labokha et al., manuscript in preparation).

It is known that Nup98 is glycosylated in vivo (Snow et al., 1987; Davis and Blobel, 1987; Finlay and Forbes, 1990). Labokha et al. showed that hydrogels formed of in vitroglycosylated Nup98 FG domain are still restrictive towards inert molecules but allow the enrichment of NTR $\cdot$ cargo complexes in a selective manner. The authors therefore assume that vertebrates titrate the density of their permeability barrier by glycosylation.

In contrast, glycosylation of FG domains is not described in yeast. A regulation of the permeability barrier by glycosylation is thus not expected. However, yeast possesses FG domains that are less-cohesive.

It seems thus reasonable to assume that yeast balanced the cohesiveness of its permeability barrier in the course of evolution by two means. First, the main constituent of the permeability barrier, an evolutionary ancestor of Nup98, was adapted resulting in three distinct homologs with partly differing properties. Second, several less-cohesive FG domains were employed to lower and balance the tightness of the barrier.

\subsection{Implications for proposed permeability barrier models}

FG domain exhibit a multitude of features. However, the significance of most of the features for functionality of the permeability barrier remained unclear. An essential feature is the binding of NTRs to FG motifs, which is not only essential for translocation but also accelerates NPC passage (Ribbeck and Görlich, 2001). It is unclear how such binding can accelerate translocation since one would expect that a binding delays passage of a molecule.

Several models have been proposed to resolve this enigma (reviewed by Terry and Wente, 2009; Wälde and Kehlenbach, 2010; Yang, 2011).

In general these models can be distinguished by their assumptions concerning the mode of interaction among FG domains. The "selective phase" model and the "forest" model explain barrier characteristics by the ability of FG domains to engage in inter-repeat contacts. On the other side, if the "entropic gate" or the "reduction of dimensionality" model faithfully described NPC selectivity, then inter-FG domain cohesion would not be required. It was recently found that in Xenopus NPCs the multivalent cohesion between FG do- 
mains is required for a functional permeability barrier (Hülsmann et al., submitted). In line with that we showed that in yeast indeed cohesiveness is an essential feature of FG domains for in vivo permeability barrier function.

In the "entropic gate" and the "reduction of dimensionality" model, translocation of NTRs only depends on the presence of NTR binding sites. The models would predict that an exchange of FG domains has no consequences as long as the inserted FG domain provides NTR binding sites.

However, we showed that not all FG domains harboring NTR binding sites can functionally replace the FG domains of Nup100p and Nup116p. On the other side, these two domains, even though they bind NTRs (Iovine et al., 1995; Bayliss et al., 2002b), cannot functionally replace the Nsp1p FG domain. The ability to replace FG domains was thus not related to NTR binding. Neither the "entropic gate" nor the "reduction of dimensionality" model can explain this observation.

The models predict different consequences concerning the deletion of FG domains. In the "reduction of dimensionality" model, deletions would have no effect as long as enough FG mass is present to line the inner wall of the channel. And the deletion of FG mass above a critical value would result in a collapse of facilitated transport. According to the "entropic gate" model, a stepwise deletion of FG domains should result in a stepwise reduction of selectivity because less FG domains would be present to repulse inert macromolecules. And like for the "reduction of dimensionality" model, the deletion of FG mass above a critical value would result in a collapse of facilitated transport. In contrast, according to the "selective phase" or the "forest" model, the effect of deletions would depend on the kind of deleted FG domains. The deletion of non-cohesive FG mass should have no effect whereas the excessive deletion of cohesive FG domains should lead to a collapse of the FG meshwork.

We observed that the simultaneous deletion of the cohesive FG domains from Nup100p and Nup116p results in lethality. Together, these FG domains provide approximately $20 \%$ of the total FG mass. If the "entropic gate" or the "reduction of dimensionality" model correctly described NPC functionality, then the deletion of more FG mass would not be compatible with viability. However, a strain with only $40 \%$ of its FG mass being left over was found to be viable.

Due to these argument, we consider the "entropic gate" and the "reduction of dimension- 
ality" model to be refuted.

On the other side, all phenotypes observed in this study are in line with predictions from the "selective phase" model and from the "forest" model. So how can we experimentally distinguish these two models?

The "selective phase" model assumes a homogenous meshwork. In contrast, the "forest" model assumes that FG domains adopt distinct "disordered structures" that create two distinct transport zones within the NPC channel. If the "forest" model correctly predicted NPC functionality, and if we simultaneously deleted all FG domains that are predicted to build up one of these transport zones, then the resulting NPC would not be functional. However, this remains to be tested.

\subsection{Perspectives}

In this study we developed a new experimental system that allows to manipulate the NPC permeability barrier in vivo. We showed that the postulated necessity of cohesiveness, according to the "selective phase" model, is indeed an essential feature of FG domains in vivo. However, we assume that both the excessive presence as well as the absence of cohesive FG mass is incompatible with the in vivo functionality of the permeability barrier. Furthermore we demonstrated that neither FG domains nor the FG domain anchor points within the NPC are equivalent. Thus, heterogeneity seems to be a crucial for in vivo NPC function.

To further analyze predictions by the "selective phase" model and to distinguish them from predictions by other models, we need to extend and exploit our system. Four main directions are obvious.

(i) So far, we did not "cleanly" generate and test all triple and quadruple combinations of FG domain deletions. Doing so would probably pave the way for the creation of new anchor domain test strains which would broaden our understanding of requirements for the anchoring of FG domains. Furthermore, we concentrated so far on the centrally localized FG domains. Is was previously shown that all peripherally localized FG domains can be deleted simultaneously in addition to the deletion of one central FG domain (Strawn et al., 2004; Terry and Wente, 2007). It is provoking to test if our triple of quadruple $\Delta \mathrm{FG}$ mutants would be viable in a background where all peripherally localized FG domains are 
deleted. A viable strain would confirm the assumption that peripherally localized FG domains mainly function in other processes than permeability barrier formation.

(ii) Our system allows to assay the consequences of fusing one FG domain to a given anchor domain. However, we are not yet able to fuse a second FG domain to an unrelated anchor domain. In order to obtain a homogenous permeability barrier, it will be crucial to exchange more than one FG domain.

(iii) To date we only tested the effect of fusing endogenous FG domains to distinct anchor domains. However, to better understand FG domain properties, it is essential to modify and evaluate existing FG domains. Maybe it is possible to balance FG domain cohesiveness in such manner that it is functional at every anchor domain. This artificial FG domain could then be characterized in detail by biophysical and biochemical methods. The artificial quadrupled FG domain of Nup145Np described in this study might serve as a useful starting point for the development of such a universally functional FG domain. It is the only domain so far that is functional at all tested anchor points. However, this artificial domain has to be further optimized since cell growth is seriously delayed when FG domains are replaced by this domain.

(iv) So far, our experimental in vivo does not allow to distinguish whether a resulting lethal phenotype of an integrated FG domain is caused (i) by a non-selective barrier that results in uncontrolled mixing of cytoplasmic and nuclear content (ii) by a barrier that is too tight so that any transport is prevented or (iii) by the blockade of one specific transport pathway.

To resolve this issue, two experimental approaches needs to be combined. We need to establish in vivo transport assays that allow to monitor the intracellular distribution of very large transport cargos (like ribosomal subunits) and of small inert molecules (like GFP). By comparing steady-state distributions of molecules in wild type strains versus strains with modified FG domains we should be able to evaluate changes in barrier tightness. However, to evaluate the cause of a lethal phenotype such transport assays need to be combined with the ability to induce changes of the barrier. If we put the gene encoding a modified FG Nup under the control of an inducible promotor, we can follow the onset of lethal effects. 


\section{Materials and Methods}

\subsection{Materials}

\subsubsection{Chemicals}

If not stated otherwise, laboratory reagents were purchased from Calbiochem (San Diego, CA, USA), GibcoBRL-Life Technologies (Paisley, UK), Merck (Darmstadt, Germany), MoBiTech (Göttingen, Germany), Pharmacia (Uppsala, Sweden), Promega (Madison, WI, USA), Roche Diagnostics (Mannheim, Germany), Carl Roth (Karlsruhe, Germany), or Sigma-Aldrich (St. Louis, MO, USA).

\subsubsection{Software}

Software

Adobe CS4

Lasergene 8

Freemind

BibTeX

ImageJ

LaTeX

Microsoft Office

Oligo 6

GeneDesigner

Papers

"Epson Scan" Software Vers. 3.28A

Mac OS X Vers. 10.6.8

\section{Website}

https://www.adobe.com/de/products/creativesuite.html http://www.dnastar.com/t-products-lasergene.aspx http://freemind.softonic.de/

http://www.bibtex.org/

http://rsbweb.nih.gov/ij/

http://www.latex-project.org/

https://office.microsoft.com

http://oligo.net/

https://www.dna20.com/genedesigner2/

http://www.mekentosj.com/papers/

http://www.epson.de/

http://www.apple.com/ 


\subsubsection{Instruments}

Research pro multi channel pipette

Set of "Pipetman" pipettes

SensoQuest lab cycler

GenePulser $^{T M}$

NanoDrop ND-2000

Biophotometer plus

Rotator SB3

Thermomixer plus

5415R cooled tabletop centrifuge

5424 tabletop centrifuge

Multifuge 3L-R with 75006445 Rotor

Liebherr Premium fridge

Liebherr Comfort freezer

Thermostatic cabinet

Ecotorn incubator

Incubator Climo-Shaker ISF1-X

Ultra low freezer

Intas Gel Documentation System

Perfection V700 Photo Scanner

Micromanipulator MSM System Series 300

BX50 Microscope

TCS SP5 microscope

iMac 2,4GHz Intel Core 2 Duo
Eppendorf AG, Hamburg, Germany

Gilson, Middleton, WI, USA

SensoQuest, Göttingen, Germany

BioRad, Hercules, CA, USA

PEQLAB Biotechnologie GmbH, Erlangen, Germany

Eppendorf AG, Hamburg, Germany

Stuart, Stone, Staffordshire, UK

Eppendorf AG, Hamburg, Germany

Eppendorf AG, Hamburg, Germany

Eppendorf AG, Hamburg, Germany

Thermo Scientific, Waltham, MA, USA

Liebherr, Biberach, Germany

Liebherr, Biberach, Germany

AquaLytic, Dortmund, Germany

Infors AG, Basel, Switzerland

Kuhner AG, Basel, Switzerland

Sanya, Osaka, Japan

Intas Science Imaging Instruments GmbH, Germany

Epson, Tokio, Japan

Singer Instruments, Roadwater, Somerset, UK

Olympus, Tokio, Japan

Leica Microsystems, Wetzlar, Germany

Apple, Cupertino, CA, UAS

\subsection{4 buffers \& solutions}

\section{$10 \times$ Ligation buffer:}

$500 \mathrm{mM}$ Tris $(\mathrm{pH} 7,5), 100 \mathrm{mM} \mathrm{MgCl}_{2}, 100 \mathrm{mM}$ DTT, $10 \mathrm{mM}$ ATP, $250 \mu \mathrm{g} / \mathrm{mL}$ BSA

\section{$10 \times$ PfuS buffer:}

$200 \mathrm{mM}$ Tris-HCl (pH9), 250mM KCl, 100mM $\left(\mathrm{NH}_{4}\right)_{2} \mathrm{SO}_{4}, 15 \mathrm{mM} \mathrm{MgSO}_{4}, 1 \%$ Tween20, $1 \mathrm{mg} / \mathrm{mL}$ BSA

\section{$10 \times$ Tth buffer:}

$100 \mathrm{mM}$ Tris- $\mathrm{HCl}(\mathrm{pH} 8,9), 15 \mathrm{mM} \mathrm{MgCl} 2,1 \mathrm{M} \mathrm{KCl}, 500 \mu \mathrm{g} / \mathrm{mL} \mathrm{BSA}, 0,5 \%$ Tween20

\section{Orange G DNA loading buffer:}

10mM Tris-HCl (pH8), 10mM EDTA (pH8), 50\% Glycerol (w/v), 0,25\% OrangeG (w/v)

\section{LiAc/TE solution:}


0,1M Lithiumacetat in $1 \times \mathrm{TE}$ buffer

$10 \times$ TE (Tris-EDTA) buffer:

100mM Tris/HCl (pH 8,0), 5mM EDTA

PEG/LiAc/TE solution:

$40 \%$ (w/v) PEG6000, 1M Lithiumacetat in $1 \times$ TE buffer

50 $\times$ TAE (Tris- Acetat-EDTA) buffer:

$242 \mathrm{~g}$ Tris base, $57,1 \mathrm{~mL}$ acetic acid, 100mL 0,5M EDTA (pH8) in total 1L

\subsubsection{Escherichia coli Media}

\section{YT liquid medium}

16g Trypton

$10 \mathrm{~g}$ Yeast Extract

$5 \mathrm{~g} \quad \mathrm{NaCl}$

$20 \mathrm{~mL}$ Glycerol 85\%

$\mathrm{H}_{2} \mathrm{O}$ up to $1 \mathrm{~L}$

\section{YT plates}

$16 \mathrm{~g}$ Trypton

$10 \mathrm{~g}$ Yeast Extract

$5 \mathrm{~g} \quad \mathrm{NaC}$

20mL Glycerol 85\%

$14 \mathrm{~g}$ Agar

$\mathrm{H}_{2} \mathrm{O}$ up to $1 \mathrm{~L}$

The ingredients were mixed and autoclaved. Before the plates were poured, $10 \mathrm{~mL} 2 \mathrm{M}$ $\mathrm{K}_{2} \mathrm{HPO}_{4}$ and $10 \mathrm{~mL}$ of $40 \%(\mathrm{w} / \mathrm{v})$ glucose were additionally added.

For the selection and culturing of bacteria after transformation, plates or liquid medium were supplemented with the appropriate antibiotics in a final concentrations of $100 \mu \mathrm{g} / \mathrm{mL}$ ampicillin or $25 \mu \mathrm{g} / \mathrm{mL}$ kanamycin.

\subsubsection{Escherichia coli Strains}

\begin{tabular}{|c|c|c|}
\hline Strain & relevante genotype & origin \\
\hline $\mathrm{NEB} 5 \alpha \mathrm{F}^{\prime}$ & 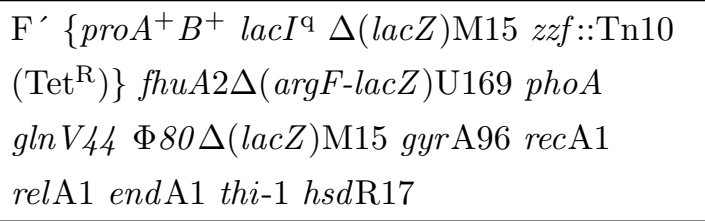 & $\begin{array}{l}\text { New England Biolabs } \\
\text { (Beverly, MA, USA) }\end{array}$ \\
\hline TOP10 F' & 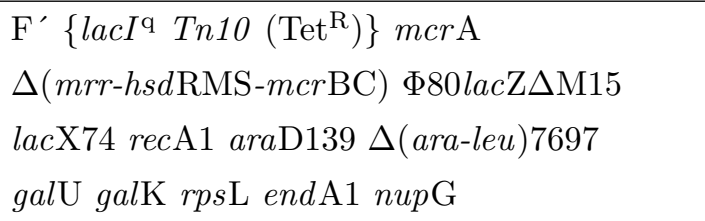 & $\begin{array}{l}\text { Invitrogen } \\
\text { (Karlsruhe, Germany) }\end{array}$ \\
\hline GM2163 & $\begin{array}{l}\mathrm{F}^{-} \text {dam-13::Tn9 dcm-6 hsdR2 mcr } \mathrm{A} \text { mcr } \mathrm{B} \\
\text { leuB6 his-4 thi-1 ara-14 lac } 1 \text { galK2 gal } 22 \\
\text { xyl-5 mtl-1 rpsL136 ton } \mathrm{A} 31 \text { tsx-78 sup } \mathrm{E} 44 \mathrm{i}\end{array}$ & Palmer and Marinus (1994) \\
\hline
\end{tabular}




\subsubsection{Saccharomyces cerevisiae Media}

Standard yeast media were used (Sherman, 2002). Plates contained 2\% (w/v) agar additionally. Media were autoclaved for sterilization and glucose was added afterwards in form of an autoclaved $40 \%(\mathrm{w} / \mathrm{v})$ glucose solution.

Agar was purchased from Invitrogen (Karlsruhe, Germany), yeast extract and tryptone from Serva (Heidelberg, Germany). CSM-mixes and yeast nitrogen base without ammonium sulfate (YNB) were obtained from MP Biomedicals (Santa Ana, CA, USA).

\section{YPD medium (yeast extract, peptone, D-glucose):}

$1 \%(\mathrm{w} / \mathrm{v})$ yeast extract, $2 \%(\mathrm{w} / \mathrm{v})$ tryptone, $2 \%(\mathrm{w} / \mathrm{v})$ glucose

\section{CSM medium (complete supplement mixture):}

$0,17 \%(\mathrm{w} / \mathrm{v})$ yeast nitrogen base without ammonium sulfate, $5 \%$ ammonium sulfate, $2 \%(\mathrm{w} / \mathrm{v})$ glucose, CSM-mix according to needs as follows:

$$
\begin{array}{rll}
\text { CSM complete } & 790 \mathrm{mg} / \mathrm{L} & (=0,079 \%(\mathrm{w} / \mathrm{v})) \\
\mathrm{CSM}-\mathrm{Leu} & 690 \mathrm{mg} / \mathrm{L} & (=0,069 \%(\mathrm{w} / \mathrm{v})) \\
\mathrm{CSM}-\mathrm{Ura} & 770 \mathrm{mg} / \mathrm{L} & (=0,077 \%(\mathrm{w} / \mathrm{v})) \\
\mathrm{CSM}-\mathrm{His} & 770 \mathrm{mg} / \mathrm{L} & (=0,077 \%(\mathrm{w} / \mathrm{v})) \\
\mathrm{CSM}-\mathrm{Ura}-\mathrm{Leu} & 670 \mathrm{mg} / \mathrm{L} & (=0,067 \%(\mathrm{w} / \mathrm{v}))
\end{array}
$$

\section{Sporulation plates:}

$1 \%(\mathrm{w} / \mathrm{v})$ potassium acetat, $0,1 \%(\mathrm{w} / \mathrm{v})$ yeast extract, $2 \%(\mathrm{w} / \mathrm{v})$ agar, $0,0204 \%(\mathrm{w} / \mathrm{v})$ adenine hemisulfate, $0,018 \%(\mathrm{w} / \mathrm{v})$ tryptophan, 0,03\% (w/v) L-leucine, 0,03\% (w/v) L-lysine. Potassium acetat, yeast extract, and agar were autoclaved before the remaining ingredients were added in form of a sterile filtered solution.

\section{FOA plates (5-fluoro-orotic- $\underline{\text { acid }})$ :}

$0,17 \%(\mathrm{w} / \mathrm{v})$ yeast nitrogen base without ammonium sulfate, $5 \%$ ammonium sulfate, $2 \%(\mathrm{w} / \mathrm{v})$ glucose, $0,079 \%(\mathrm{w} / \mathrm{v})$ CSM complete mix, $1 \%(\mathrm{w} / \mathrm{v})$ FOA, $2 \%(\mathrm{w} / \mathrm{v})$ agar.

Yeast nitrogen base without ammonium sulfate, ammonium sulfate, CSM complete mix, and agar were prepared and autoclaved. After the solution was cooled down to $\sim 50^{\circ} \mathrm{C}$, glucose in form of a $40 \%(\mathrm{w} / \mathrm{v})$ autoclaved solution and $100 \times \mathrm{FOA}$ solution in DMSO 
(Zymo Research, Irvine, CA, USA) were added. Before the plates were poured, the pH was adjusted to $\sim 4,4$ by addition of $10 \mathrm{M} \mathrm{NaOH}(\sim 100 \mu \mathrm{L}$ for $500 \mathrm{~mL}$ of medium).

\subsubsection{Saccharomyces cerevisiae Strains}

All Saccharomyces cerevisiae strains in this study originate from a W303 strain background (originally constructed by Rodney Rothstein).

Table 4.1: S. cerevisiae strains used in this study. Starting with strain YMR35, all strains below are MATa $\triangle$ ade2 his3-11, 15 leu2-3, 112 LYS2 trp1-1 ura3 can1-100 and were generated as described in 4.2.2.3 and 4.2.2.4. Please note that numbers given in parentheses indicate deleted amino acids compared to the wild type protein.

\begin{tabular}{|c|c|c|}
\hline Strain & Relevante genotype & $\begin{array}{l}\text { Parental strain / } \\
\text { Reference }\end{array}$ \\
\hline RJY358 & MATa ade2-1 his3-11, 15 leu2-3, 112 LYS2 trp1-1 ura3 can1-100 & $\begin{array}{l}\text { provided by } \\
\text { R.P. Jansen }\end{array}$ \\
\hline SFY401 & MATa ADE2 his3-11, 15 leu2-3, 112 LYS2 trp1-1 ura3 can1-100 & $\begin{array}{l}\text { RJY358 / provided by } \\
\text { S. Frey }\end{array}$ \\
\hline SWY2284 & $\begin{array}{l}\text { MAT } \alpha \text { ade2-1::ADE2 his3-11, } 15 \text { leu2-3, } 112 \text { LYS2 trp1-1 ura3-1 } \\
\text { can1-100 }\end{array}$ & $\begin{array}{l}\text { provided by } \\
\text { S.R. Wente (Strawn } \\
\text { et al., 2004) }\end{array}$ \\
\hline SWY2885 & $\begin{array}{l}\text { MATa/a myc-loxP-nup } 57 \Delta G L F G / \text { myc-loxP-nup } 57 \Delta G L F G \text { t7- } \\
\text { loxP-nup } 49 \Delta G L F G / t 7-l o x P-n u p 49 \Delta G L F G \text { ade2-1::ADE2/ade2- } \\
\text { 1::ADE2 his3/his3 leu2/leu2 LYS2/lys2 trp1-1/TRP1 ura3/ura3 } \\
\text { can1-100/can1-100 }\end{array}$ & $\begin{array}{l}\text { provided by } \\
\text { S.R. Wente (Strawn } \\
\text { et al., 2004) }\end{array}$ \\
\hline SWY2930 & 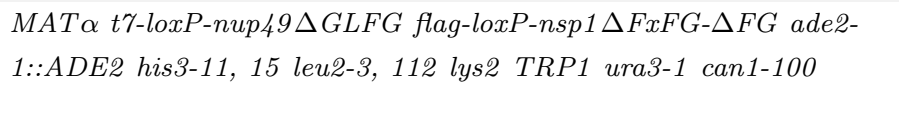 & $\begin{array}{l}\text { provided by } \\
\text { S.R. Wente (Strawn } \\
\text { et al., 2004) }\end{array}$ \\
\hline SWY2934 & $\begin{array}{l}\text { MATa myc-loxP-nup } 57 \Delta G L F G \text { flag-loxP-nsp1 } \Delta F x F G-\triangle F G \text { ade2- } \\
1:: A D E 2 \text { his3-11, } 15 \text { leu2-3, } 112 \text { lys2 TRP1 ura3-1 can1-100 }\end{array}$ & $\begin{array}{l}\text { provided by } \\
\text { S.R. Wente (Strawn } \\
\text { et al., 2004) }\end{array}$ \\
\hline YMR18 & $\begin{array}{l}\text { myc-loxP-nup } 57 \Delta G L F G / \text { myc-loxP-nup } 57 \Delta G L F G \text { t7-loxP- } \\
\text { nup } 49 \Delta G L F G / t 7 \text {-loxP-nup } 49 \Delta G L F G \text { NSP1/Dnsp } 1: \text { :HIS3 }\end{array}$ & SWY2885 \\
\hline YMR26.69 & $\begin{array}{l}\text { myc-loxP-nup57 } 6 \text { GLFG t7-loxP-nup49DGLFG } \triangle \text { nsp1::HIS3 + } \\
\text { pMR69 (NSP1 NUP49 NUP57 URA3 CEN) }\end{array}$ & YMR18 \\
\hline YMR26.109 & $\begin{array}{l}\text { myc-loxP-nup57 } 5 L F G \text { t7-loxP-nup49DGLFG } \Delta \text { nsp1::HIS3 + } \\
\text { pMR109 (ADH1promotor NSP1 URA3 CENmut) }\end{array}$ & YMR18 \\
\hline YMR27 & $\begin{array}{l}\text { myc-loxP-nup } 57 \Delta G L F G \text { flag-loxP-nsp } 1 \Delta F X F G-\triangle F G \Delta \text { nup } 49:: H I S \\
+ \text { pMR62 (ADH1promotor NUP49 URA3 CEN) }\end{array}$ & SWY2934 \\
\hline YMR28 & $\begin{array}{l}\text { t7-loxP-nup } 49 \Delta G L F G \text { flag-loxP-nsp1 } F X F G-\triangle F G \Delta \text { nup } 57:: H I S \\
+ \text { pMR63 (ADH1promotor NUP57 URA3 CEN) }\end{array}$ & SWY2930 \\
\hline YMR29 & 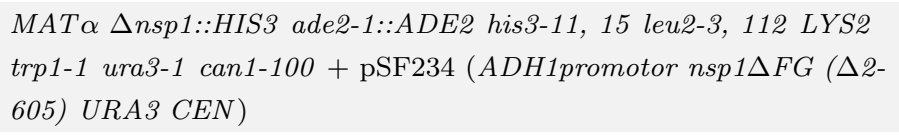 & SWY2284 \\
\hline YMR30 & $\begin{array}{l}\text { MAT } \alpha \text { nup } 49:: H I S 3 \text { ade2-1::ADE2 his3-11, } 15 \text { leu2-3, } 112 \text { LYS2 } \\
\text { trp1-1 ura3-1 can1-100 + pMR62 (ADH1promotor NUP49 URA3 } \\
\text { CEN) }\end{array}$ & SWY2284 \\
\hline
\end{tabular}


Table 4.1 - Continued from previous page

\begin{tabular}{|c|c|c|}
\hline Strain & Relevante genotype & $\begin{array}{l}\text { Parental strain / } \\
\text { Reference }\end{array}$ \\
\hline YMR31 & $\begin{array}{l}\text { MAT } \triangle \text { nup57::HIS3 ade2-1::ADE2 his3-11, } 15 \text { leu2-3, 112 LYS2 } \\
\text { trp1-1 ura3-1 can1-100 + pMR63 (ADH1promotor NUP57 URA3 } \\
\text { CEN) }\end{array}$ & SWY2284 \\
\hline YMR35 & MATa $\Delta$ ade2 his3-11, 15 leu2-3, 112 LYS2 trp1-1 ura3 can1-100 & SFY401 \\
\hline YMR36 & $\Delta$ nsp1::HIS3-ADE2 + pMR61 (ADH1promotor NSP1 URA3 CEN) & YMR35 \\
\hline YMR37 & $\triangle$ nup1::URA3-ADE2 & YMR35 \\
\hline YMR38 & $\triangle$ nup2::URA3-ADE2 & YMR35 \\
\hline YMR39 & $\triangle$ nup42::URA3-ADE2 & YMR35 \\
\hline YMR40 & $\triangle$ nup60::URA3-ADE2 & YMR35 \\
\hline YMR41 & $\begin{array}{l}\triangle \text { nup 49::HIS3-ADE2 + pMR62 (ADH1promotor NUP49 URA3 } \\
\text { CEN) }\end{array}$ & YMR35 \\
\hline YMR42 & $\begin{array}{l}\triangle \text { nup } 57:: H I S 3-A D E 2+\text { pMR63 (ADH1promotor NUP57 URA3 } \\
\text { CEN) }\end{array}$ & YMR35 \\
\hline YMR43 & $\begin{array}{l}\triangle \text { nup 145::HIS3-ADE2 + pSF1126 (ADH1promotor NUP145 URA3 } \\
C E N)\end{array}$ & YMR35 \\
\hline YMR44 & $\triangle$ nup 100::URA3-ADE2 & YMR35 \\
\hline YMR45 & $\begin{array}{l}\triangle \text { nup 116::HIS3-ADE2 + pSF1127 (ADH1promotor NUP116 URA3 } \\
C E N)\end{array}$ & YMR35 \\
\hline YMR46 & $\begin{array}{l}\triangle \text { nup 159::HIS3-ADE2 + pSF1128 (ADH1promotor NUP159 URA3 } \\
C E N)\end{array}$ & YMR35 \\
\hline YMR47 & nup $42 \Delta F G(\Delta 4-364)$ & YMR39 \\
\hline YMR48 & nup $1 \Delta F G(\Delta 382-888)$ & YMR37 \\
\hline YMR49 & nup $1 \Delta F G(\Delta 384-1043)$ & YMR37 \\
\hline YMR50 & nup 42 $\Delta F G(\Delta 4-364) \triangle$ nup $1:: U R A 3-A D E 2$ & YMR47 \\
\hline YMR51 & nup 42 $\Delta F G(\Delta 4-364) \Delta$ nup2::URA3-ADE2 & YMR47 \\
\hline YMR52 & nup $42 \Delta F G(\Delta 4-364) \Delta$ nup60::URA3-ADE2 & YMR47 \\
\hline YMR53 & nup42 $\Delta F G(\Delta 4-364) \Delta$ nup 100::URA3-ADE2 & YMR47 \\
\hline YMR54 & $\begin{array}{l}\triangle n s p 1:: H I S 3-A D E 2+\text { pMR109 (ADH1promotor NSP1 URA3 } \\
\text { CENmut) }\end{array}$ & YMR35 \\
\hline YMR55 & $\begin{array}{l}\text { Dnup 49::HIS3-ADE2 + pMR114 (ADH1promotor NUP49 URA3 } \\
\text { CENmut) }\end{array}$ & YMR35 \\
\hline YMR56 & $\begin{array}{l}\text { Anup57::HIS3-ADE2 + pMR115 (ADH1promotor NUP57 URA3 } \\
\text { CENmut) }\end{array}$ & YMR35 \\
\hline YMR57 & $\begin{array}{l}\triangle \text { nup 145::HIS3-ADE2 + pMR116 (ADH1promotor NUP145 URA3 } \\
\text { CENmut) }\end{array}$ & YMR35 \\
\hline YMR58 & $\begin{array}{l}\text { Anup116::HIS3-ADE2 + pMR117 (ADH1promotor NUP116 URA3 } \\
\text { CENmut) }\end{array}$ & YMR35 \\
\hline YMR60 & nup1 $\Delta F G(\Delta 382-888) \triangle$ nup2::URA3-ADE2 & YMR48 \\
\hline YMR61 & nup1 $\Delta F G(\Delta 382-888) \Delta$ nup 42::URA3-ADE2 & YMR48 \\
\hline YMR62 & nup1 $\Delta F G(\Delta 382-888) \Delta$ nup $60:: U R A 3-A D E 2$ & YMR48 \\
\hline YMR63 & nup1 $\Delta F G(\Delta 382-888) \Delta$ nup100::URA3-ADE2 & YMR48 \\
\hline YMR64 & nsp $1 \Delta F G(\Delta 3-602)$ nup $57 \Delta F G(\Delta 2-223)$ nuр $145 \Delta F G(\Delta 10-209)$ & YMR108 \\
\hline YMR65 & nup1 $\Delta F G(\Delta 384-1043) \triangle$ nup42::URA3-ADE2 & YMR49 \\
\hline YMR66 & nup 100 $\Delta F G(\Delta 2-576)$ nup $145 \Delta F G(\Delta 10-209)$ nsp $1:: N S P 1-G L E B S^{\mathbf{a}}$ & YMR116 \\
\hline YMR67 & nup1 $\Delta F G(\Delta 384-1043) \Delta$ nup100::URA3-ADE2 & YMR49 \\
\hline
\end{tabular}


Table 4.1 - Continued from previous page

\begin{tabular}{|c|c|c|}
\hline Strain & Relevante genotype & $\begin{array}{l}\text { Parental strain / } \\
\text { Reference }\end{array}$ \\
\hline YMR68 & $\begin{array}{l}\text { nsp1 } \Delta F G(\Delta 3-602) \text { nup } 57 \Delta F G(\Delta 2-223) \text { nup } 145 \Delta F G(\Delta 10-209) \\
\Delta \text { nup49::HIS3-ADE2 + pMR114 (ADH1promotor NUP49 URA3 } \\
\text { CENmut) }\end{array}$ & YMR64 \\
\hline YMR69 & nup $60 \Delta F G(\Delta 397-512)$ & YMR40 \\
\hline YMR70 & $n s p 1:: N S P 1-G L E B S^{\mathbf{b}}$ & YMR35 \\
\hline YMR71 & nup $1 \Delta F G(\Delta 382-888)$ nup $2 \Delta F G(\Delta 189-556)$ & YMR60 \\
\hline YMR72 & $n u p 49 \Delta F G(\Delta 2-236)$ & YMR55 \\
\hline YMR73 & $\begin{array}{l}\text { nsp1::NSP1-GLEBS } S^{\mathbf{b}} \Delta \text { nup116::HIS3-ADE2 + pMR117 } \\
(\text { ADH1promotor NUP116 URA3 CENmut) }\end{array}$ & YMR70 \\
\hline YMR74 & nsp $1:: N S P 1-G L E B S^{\mathbf{b}}$ nup $100 \Delta F G(\Delta 2-576)$ & YMR70 \\
\hline YMR75 & $n s p 1:: N S P 1-G L E B S^{\mathbf{b}}$ nup $145 \Delta F G(\Delta 10-209)$ & YMR102 \\
\hline YMR76 & nup $1 \Delta F G(\Delta 382-1043)$ nup $42 \Delta F G(\Delta 4-364)$ & YMR65 \\
\hline YMR77 & nsp1::NSP1-GLEBS ${ }^{\mathbf{b}}$ nup $145 \Delta F G(\Delta 10-209)$ nup116 $F G(\Delta 2-714)$ & YMR75 \\
\hline YMR78 & $\begin{array}{l}\text { nsp1 } \Delta F G(\Delta 3-602) \text { nup } 49 \Delta F G(\Delta 2-236) \text { nup } 145 \Delta F G(\Delta 10-209) \\
\Delta \text { nup 57::HIS3-ADE2 }+\operatorname{pMR} 115(\text { ADH1promotor NUP57 URA3 } \\
\text { CENmut })\end{array}$ & YMR120 \\
\hline YMR79 & nup $1 \Delta F G(\Delta 382-888)$ nup $42 \Delta F G(\Delta 4-364)$ & YMR50 \\
\hline YMR80 & nup $49 \Delta F G(\Delta 2-236) \Delta$ nup100::URA3-ADE2 & YMR72 \\
\hline YMR81 & $\begin{array}{l}\text { nsp1 } \Delta F G(\Delta 3-602) \text { nup } 49 \Delta F G(\Delta 2-236) \text { nup } 100 \Delta F G(\Delta 2-576) \\
\Delta \text { nup 57::HIS3-ADE2 + pMR115 (ADH1promotor NUP57 URA3 } \\
\text { CENmut })\end{array}$ & YMR118 \\
\hline YMR82 & $\begin{array}{l}\text { nsp1 } \Delta F G(\Delta 3-602) \text { nup } 49 \Delta F G(\Delta 2-236) \text { nup } 100 \Delta F G(\Delta 2-576) \\
\Delta \text { nup145::HIS3-ADE2 }+ \text { pMR116 (ADH1promotor NUP145 URA3 } \\
\text { CENmut })\end{array}$ & YMR118 \\
\hline YMR83 & $\begin{array}{l}\text { nsp1 } \Delta F G(\Delta 3-602) \text { nup } 49 \Delta F G(\Delta 2-236) \text { nup } 57 \Delta F G(\Delta 2-223) \\
\Delta \text { nup100::HIS3-ADE2 + pMR151 (ADH1promotor NUP100 URA3 } \\
\text { CENmut) }\end{array}$ & YMR119 \\
\hline YMR84 & $\begin{array}{l}\text { nsp1 } \Delta F G(\Delta 3-602) \text { nup } 49 \Delta F G(\Delta 2-236) \text { nup } 100 \Delta F G(\Delta 2-576) \\
\Delta \text { nup116::HIS3-ADE2 }+ \text { pMR117 (ADH1promotor NUP116 URA3 } \\
\text { CENmut })\end{array}$ & YMR118 \\
\hline YMR85 & $\begin{array}{l}\text { nsp1 } \Delta F G(\Delta 3-602) \text { nup } 49 \Delta F G(\Delta 2-236) \text { nup } 57 \Delta F G(\Delta 2-223) \\
\Delta \text { nup145::HIS3-ADE2 }+ \text { pMR116 }(\text { ADH1promotor NUP145 URA3 } \\
\text { CENmut })\end{array}$ & YMR119 \\
\hline YMR86 & nup60 $\Delta F G(\Delta 397-512) \Delta$ nup2::URA3-ADE2 & YMR69 \\
\hline YMR87 & nup60 $\Delta F G(\Delta 397-512) \Delta$ nup42::URA3-ADE2 & YMR69 \\
\hline YMR88 & nup60 $\Delta F G(\Delta 397-512) \Delta$ nup100::URA3-ADE2 & YMR69 \\
\hline YMR89 & nup1 $\Delta F G(\Delta 382-888)$ nup2 $\Delta F G(\Delta 189-556) \Delta$ nup 42::URA3-ADE2 & YMR71 \\
\hline YMR90 & nup1 $\Delta F G(\Delta 382-888)$ nup2 $\Delta F G(\Delta 189-556) \Delta$ nup60::URA3-ADE2 & YMR71 \\
\hline YMR91 & $\begin{array}{l}\text { nup1 } \Delta F G(\Delta 382-888) \text { nup2 } \Delta F G(\Delta 189-556) \Delta \text { nup } 100:: U R A 3- \\
\text { ADE2 }\end{array}$ & YMR71 \\
\hline YMR92 & $\begin{array}{l}\text { nup1 } \Delta F G(\Delta 382-1043) \text { nup42 } \Delta F G(\Delta 4-364) \Delta \text { nup } 100:: U R A 3- \\
\text { ADE2 }\end{array}$ & YMR76 \\
\hline YMR93 & nup1 $\Delta F G(\Delta 382-888)$ nup 42 $\Delta F G(\Delta 4-364) \Delta$ nup2::URA3-ADE2 & YMR79 \\
\hline YMR94 & nup1 $\Delta F G(\Delta 382-888)$ nup $42 \Delta F G(\Delta 4-364) \Delta$ nup60::URA3-ADE2 & YMR79 \\
\hline YMR95 & nup1 $\Delta F G(\Delta 382-888)$ nup 42 $\Delta F G(\Delta 4-364) \Delta$ nup100::URA3-ADE2 & YMR79 \\
\hline
\end{tabular}


Table 4.1 - Continued from previous page

\begin{tabular}{|c|c|c|}
\hline Strain & Relevante genotype & $\begin{array}{l}\text { Parental strain / } \\
\text { Reference }\end{array}$ \\
\hline YMR96 & nup1 $\Delta F G(\Delta 382-1043)$ nup 42 $\Delta F G(\Delta 4-364) \Delta$ nup60::URA3-ADE2 & YMR76 \\
\hline YMR97 & $\begin{array}{l}n u p 49 \Delta F G(\Delta 2-236) \Delta n s p 1:: H I S 3-A D E 2+\text { pMR109 } \\
\text { (ADH1promotor NSP1 URA3 CENmut) }\end{array}$ & YMR72 \\
\hline YMR98 & $\begin{array}{l}\text { nup } 49 \Delta F G(\Delta 2-236) \Delta \text { nup } 159:: H I S 3-A D E 2+\text { pMR118 } \\
\text { (ADH1promotor NUP159 URA3 CENmut) }\end{array}$ & YMR72 \\
\hline YMR99 & $\begin{array}{l}\text { nup } 49 \Delta F G(\Delta 2-236) \triangle \text { nup } 145:: H I S 3-A D E 2+\text { pMR } 116 \\
\text { (ADH1promotor NUP145 URA3 CENmut) }\end{array}$ & YMR72 \\
\hline YMR100 & $n s p 1 \Delta F G(\Delta 3-602)$ & YMR35 \\
\hline YMR101 & nup $57 \Delta F G(\Delta 2-223) \mathrm{H} 107 \mathrm{Q}$ & YMR35 \\
\hline YMR102 & nup145 $\Delta F G(\Delta 10-209)$ & YMR35 \\
\hline YMR103 & nup $49 \Delta F G(\Delta 2-236)$ nup $145 \Delta F G(\Delta 10-209)$ & YMR72 \\
\hline YMR104 & $n s p 1 \Delta F G(\Delta 3-602)$ nup $57 \Delta F G(\Delta 2-223)$ & YMR100 \\
\hline YMR105 & $n s p 1 \Delta F G(\Delta 3-602)$ nup $145 \Delta F G(\Delta 10-209)$ & YMR100 \\
\hline YMR106 & $n s p 1 \Delta F G(\Delta 3-602)$ nup $49 \Delta F G(\Delta 2-236)$ & YMR72 \\
\hline YMR107 & nup 49 $\Delta F G(\Delta 2-236)$ nup $57 \Delta F G(\Delta 2-223)$ & YMR72 \\
\hline YMR108 & nup $57 \Delta F G(\Delta 2-223)$ nup $145 \Delta F G(\Delta 10-209)$ & YMR102 \\
\hline YMR109 & nup $49 \Delta F G(\Delta 2-236)$ nup $57 \Delta F G(\Delta 2-223)$ nup $145 \Delta F G(\Delta 10-209)$ & YMR103 \\
\hline YMR110 & $\begin{array}{l}\text { nup } 49 \Delta F G(\Delta 2-236) \text { nup } 145 \Delta F G(\Delta 10-209) \Delta \text { nsp } 1: \text { HIS3-ADE2 + } \\
\text { pMR109 (ADH1promotor NSP1 URA3 CENmut) }\end{array}$ & YMR103 \\
\hline YMR111 & $\begin{array}{l}\text { nup } 49 \Delta F G(\Delta 2-236) \text { nup145 } \Delta F G(\Delta 10-209) \Delta \text { nup } 57:: H I S 3-A D E 2 \\
+ \text { pMR115 (ADH1promotor NUP57 URA3 CENmut) }\end{array}$ & YMR103 \\
\hline YMR112 & $\begin{array}{l}\text { nsp1 } \Delta F G(\Delta 3-602) \text { nup } 145 \Delta F G(\Delta 10-209) \Delta \text { nup } 49:: H I S 3-A D E 2+ \\
\text { pMR114 (ADH1promotor NUP49 URA3 CENmut) }\end{array}$ & YMR105 \\
\hline YMR113 & $\begin{array}{l}\text { nup } 49 \Delta F G(\Delta 2-236) \text { nup } 57 \Delta F G(\Delta 2-223) \text { nup } 145 \Delta F G(\Delta 10-209) \\
\Delta \text { nsp1::HIS3-ADE2 + pMR109 }(\text { ADH1promotor NSP1 URA3 } \\
\text { CENmut })\end{array}$ & YMR109 \\
\hline YMR114 & $\begin{array}{l}\text { nsp1 } \Delta F G(\Delta 3-602) \text { nup145 } \Delta F G(\Delta 10-209) \Delta \text { nup } 57:: H I S 3-A D E 2 \\
+ \text { pMR115 (ADH1promotor NUP57 URA3 CENmut) }\end{array}$ & YMR105 \\
\hline YMR115 & $n s p 1 \Delta F G(\Delta 3-602)$ nup $100 \Delta F G(\Delta 2-576)$ & YMR100 \\
\hline YMR116 & nup100 $\Delta F G(\Delta 2-576)$ nup $145 \Delta F G(\Delta 10-209)$ & YMR102 \\
\hline YMR117 & $n s p 1 \Delta F G(\Delta 3-602)$ nup $57 \Delta F G(\Delta 2-223)$ nup $100 \Delta F G(\Delta 2-576)$ & YMR115 \\
\hline YMR118 & $n s p 1 \Delta F G(\Delta 3-602)$ nup $49 \Delta F G(\Delta 2-236)$ nup $100 \Delta F G(\Delta 2-576)$ & YMR106 \\
\hline YMR119 & $n s p 1 \Delta F G(\Delta 3-602)$ nuр $49 \Delta F G(\Delta 2-236)$ nup $57 \Delta F G(\Delta 2-223)$ & YMR106 \\
\hline YMR120 & $n s p 1 \Delta F G(\Delta 3-602)$ nup $49 \Delta F G(\Delta 2-236)$ nup $145 \Delta F G(\Delta 10-209)$ & YMR103 \\
\hline YMR121 & $\begin{array}{l}\text { nup } 49 \Delta F G(\Delta 2-236) \text { nup } 57 \Delta F G(\Delta 2-223) \text { nup } 145 \Delta F G(\Delta 10-209) \\
\Delta \text { nup } 100:: H I S 3-A D E 2+\operatorname{pMR} 151(\text { ADH1promotor NUP100 URA3 } \\
\text { CENmut) }\end{array}$ & YMR109 \\
\hline YMR122 & $\begin{array}{l}n s p 1 \Delta F G(\Delta 3-602) \text { nup } 49 \Delta F G(\Delta 2-236) \text { nup } 100 \Delta F G(\Delta 2-576) \\
n u p 145 \Delta F G(\Delta 10-209)\end{array}$ & YMR118 \\
\hline YMR123 & $\begin{array}{l}n s p 1 \Delta F G(\Delta 3-602) \text { nup } 49 \Delta F G(\Delta 2-236) \text { nup } 57 \Delta F G(\Delta 2-223) \\
\text { nup100 } \Delta F G(\Delta 2-576)\end{array}$ & YMR118 \\
\hline YMR124 & $\begin{array}{l}\text { nsp1 } \Delta F G(\Delta 3-602) \text { nup49 } \Delta F G(\Delta 2-236) \text { nup } 57 \Delta F G(\Delta 2- \\
\text { 223) nup100 } \Delta F G(\Delta 2-576) \Delta \text { nup116::HIS3-ADE2 }+ \text { pMR117 } \\
\text { (ADH1promotor NUP116 URA3 CENmut) }\end{array}$ & YMR123 \\
\hline
\end{tabular}




\begin{tabular}{|c|c|c|}
\hline Strain & Relevante genotype & $\begin{array}{l}\text { Parental strain / } \\
\text { Reference }\end{array}$ \\
\hline YMR125 & $\begin{array}{l}\text { nsp1 } \Delta F G(\Delta 3-602) \text { nup } 49 \Delta F G(\Delta 2-236) \text { nup100 } \Delta F G(\Delta 2- \\
576) \text { nup145 } F G(\Delta 10-209) \Delta \text { nup } 57:: H I S 3-A D E 2+\text { pMR115 } \\
\text { (ADH1promotor NUP57 URA3 CENmut })\end{array}$ & YMR122 \\
\hline YMR127 & $\begin{array}{l}\text { nsp1::NSP1-GLEBS } S^{\mathbf{b}} \text { nup100 } \triangle F G(\triangle 2-576) \triangle \text { nup116::HIS3-ADE2 } \\
\text { + pMR117 (ADH1promotor NUP116 URA3 CENmut) }\end{array}$ & YMR74 \\
\hline YMR128 & $\begin{array}{l}\text { nsp1::NSP1-GLEBS } S^{\mathbf{b}} \text { nup145 } \mathrm{FG}(\Delta 10-209) \text { nup116 } \Delta F G(\Delta 2-714) \\
\Delta \text { nup100::HIS3-ADE2 + pMR151 (ADH1promotor NUP100 URA3 } \\
\text { CENmut) }\end{array}$ & YMR77 \\
\hline \multicolumn{3}{|c|}{ "CEN mut" indicates a vector with mutated centromere region. See 4.1 .10 for details. } \\
\hline \multicolumn{3}{|c|}{ a The GLEBS domain of Nup116p (amino acids 105-175) (Bailer et al., 1998) was inserted into the FG domain } \\
\hline \multicolumn{3}{|c|}{ of Nsp1p between amino acids 271 and 273. The insertion resulted in the following exact amino acid sequence: } \\
\hline \multicolumn{3}{|c|}{ Nsp1p 1-271 + KL + Nup116p 105-175 + GLR + Nsp1p 273-823. } \\
\hline \multicolumn{3}{|c|}{ b In this strain the GLEBS domain insertion resulted in the following exact amino acid sequence: } \\
\hline \multicolumn{3}{|c|}{ Nsp1p 1-271 + KL + Nup116p 105-175 + GLR + Nsp1p 273-602 + T + Nsp1p 603-823. } \\
\hline
\end{tabular}

\subsubsection{Oligonucleotides}

Oligonucleotides were synthesized by Sigma-Aldrich (St. Louis, MO, USA), Eurofins MWG GmbH (Ebersberg, Germany), or BioSpring GmbH (Frankfurt a. M., Germany).

\subsubsection{Plasmids}

Plasmids were generated as described in section 4.2.1. Plasmids characterized with "mutated $C E N$ " or "CENmut" originate from the pRS416 plasmid backbone. In these plasmids, the centromere region was mutated to result in a less mitotically stable replication plasmid by deleting the 24 base pairs between the $P m l$ I and the $S w a$ I endonuclease recognition sites of the $\mathrm{ARSH}_{4}$ sequence.

The plasmid backbone "pRS402mod" originates from pRS402 but carries additional the URA3 gene sub-cloned from pRS406.

Plasmids named pSF were kindly provided by Steffen Frey.

The following upstream and downstream regions were used as promotor and terminator regions respectively: 


\begin{tabular}{l|c|c} 
& $\begin{array}{c}\text { Promotor } \\
\text { (bp before start codon) }\end{array}$ & $\begin{array}{c}\text { Terminator } \\
\text { (bp after stop codon) }\end{array}$ \\
\hline ADH1 & 433 & - \\
NUF2 & - & 306 \\
NSP1 & 488 & 441 \\
NUP & 563 & 401 \\
NUP2 & 439 & 595 \\
NUP42 & 500 & 303 \\
NUP49 & 698 & 298 \\
NUP57 & 456 & 395 \\
NUP60 & 561 & 525 \\
NUP100 & 300 & 300 \\
NUP116 & 585 & 425 \\
NUP145 & 416 & 406 \\
NUP159 & 616 & 721
\end{tabular}

Plasmids used in FOA Assays are listed in table 4.2. All other plasmids used in this study are listed in table 4.3 .

Table 4.2: Plasmids used in FOA Assays Plasmids listed in this table originate from pRS415 (CEN LEU2 Amp). All genes are flanked by the endogenous promotor and terminator regions according to the coded anchor domain.

\begin{tabular}{lll}
\hline Plasmid & Insert & Coded protein sequence \\
\hline pMR001 & Nsp1-anchor & Nsp1p 1-2 + GSTQA + 603-823 \\
pMR002 & Nsp1-FGs Nsp1-anchor & Nsp1p 1-2 + GS + 2-602 + T + 603-823 \\
pMR003 & Nup100-FGs Nsp1-anchor & Nsp1p 1-2 + GS + Nup100p 2-580 + T + Nsp1p 603-823 \\
pMR004 & Nup116-FGs Nsp1-anchor & Nsp1p 1-2 + GS + Nup116p 2-725 + A + Nsp1p 603-823 \\
pMR005 & Nup1-FxFGs Nsp1-anchor & Nsp1p 1-2 + GS + Nup1p 384-888 + T + Nsp1p 603-823 \\
pMR006 & Nup2-FxFGs Nsp1-anchor & Nsp1p 1-2 + GS + Nup2p 189-527 + T + Nsp1p 603-823 \\
pMR015 & Nup49-GLFG Nsp1-anchor & Nsp1p 1-2 + GS + Nup49p 2-236 + T + Nsp1p 603-823 \\
pMR016 & Nup57-GLFG Nsp1-anchor & Nsp1p 1-2 + GS + Nup57p 2-223 + A + Nsp1p 603-823 \\
pMR019 & Nup145N-GLFG Nsp1anchor & Nsp1p 1-2 + GS + Nup145Np 10-209 + A + Nsp1p 603-823 \\
pMR020 & Nup42-FG Nsp1anchor & Nsp1p 1-2 + GS + Nup42p 4-364 + A + Nsp1p 603-823 \\
pMR021 & Nsp1 & Nsp1p wild type \\
pMR023 & empty & - \\
pMR024 & Nup159-FG Nsp1anchor & Nsp1p 1-2 + GS + Nup159p 464-876 + A + Nsp1p 603-823 \\
pMR025 & Nup60-FxF Nsp1anchor & Nsp1p 1-2 + GS + Nup60p 397-512 + A + Nsp1p 603-823 \\
pMR060 & Nup57anchor-Scar (myc loxP) & MCEQKLISEEDLTSTTLNITSYNVCYTKLLGDIRST + \\
& & Nup57p 224-541 \\
pMR068 & Nup49anchor-Scar (T7 loxP) & MMASMTGGQQMGTSTTLNITSYNVCYTKLLGDIRST + \\
& & Nup49p 237-472 \\
pMR070 & Nup49anchor & MFGSTQAS + Nup49p 237-472 \\
pMR071 & Nup57anchor & M GSTQAS + Nup57p 224-541 \\
pMR076 & Nup100 & Nup100p wild type \\
\hline
\end{tabular}


Table 4.2 - Continued from previous page

\begin{tabular}{|c|c|c|}
\hline Plasmid & Insert & Coded protein sequence \\
\hline pMR082 & Nup116 & Nup116p wild type \\
\hline pMR084 & Citrulin-Nsp1anchor & NGSTQAS + Citrulin + GSGP + Nsp1p 603-823 \\
\hline pMR085 & Citrulin-Nup49anchor & MFGSTQAS + Citrulin + GSGPS + Nup49p 237-472 \\
\hline pMR086 & Citrulin-Nup57anchor & M GSTQAS + Citrulin + Nup57p 224-541 \\
\hline pMR087 & Nsp1FG-Citrulin-Nsp1anchor & $\begin{array}{l}\text { Nsp1p 1-2 + GS + Nsp1p 2-602 + TS + Citrulin + GSGP + } \\
\text { Nsp1p 603-823 }\end{array}$ \\
\hline pMR088 & $\begin{array}{l}\text { Nup49GLFG-Citrulin- } \\
\text { Nup49anchor }\end{array}$ & $\begin{array}{l}\text { MFGS + Nup49p 2-236 + TS + Citrulin + GSGPS + Nup49p } \\
237-472\end{array}$ \\
\hline pMR089 & $\begin{array}{l}\text { Nup57GLFG-Citrulin- } \\
\text { Nup57anchor }\end{array}$ & $\begin{array}{l}\text { MNGS + Nup57p 2-223 + AS + Citrulin + GSGPS + } \\
\text { Nup57p 224-541 }\end{array}$ \\
\hline pMR110 & $\begin{array}{l}\text { Nup100GLFG-Citrulin- } \\
\text { Nsplanchor }\end{array}$ & $\begin{array}{l}\text { MNGS + Nup100p 2-580 + TS + Citrulin + GSGP + Nsp1p } \\
603-823\end{array}$ \\
\hline pMR111 & $\begin{array}{l}\text { Nup100GLFG-Citrulin- } \\
\text { Nup49anchor }\end{array}$ & $\begin{array}{l}\text { MFGS + Nup100p 2-580 + TS + Citrulin + GSGPS + } \\
\text { Nup49p 237-472 }\end{array}$ \\
\hline pMR112 & $\begin{array}{l}\text { Nup100GLFG-Citrulin- } \\
\text { Nup57anchor }\end{array}$ & $\begin{array}{l}\text { MNGS + Nup100p 2-580 + TS + Citrulin + GSGPS + } \\
\text { Nup57p 224-541 }\end{array}$ \\
\hline pMR136 & Nup100anchor & Nup100p 1 + GSTQAS + Nup100p 571-959 \\
\hline pMR155 & Nup49-GLFG Nup100anchor & Nup100p $1+$ GS + Nup49p 2-236 + TS + Nup100p 571-959 \\
\hline pMR156 & Nup57-GLFG Nup100anchor & Nup100p $1+$ GS + Nup57p 2-223 + AS + Nup100p 571-959 \\
\hline pMR157 & Nsp1-FG Nup100anchor & Nup100p $1+$ GS + Nsp1p 2-602 + TS + Nup100p 571-959 \\
\hline pMR160 & Nup1-FxFG Nup100anchor & Nup100p $1+$ GS + Nup1p 384-888 + TS + Nup100p 571-959 \\
\hline pMR161 & Nup2-FxFG Nup100anchor & Nup100p $1+$ GS + Nup2p 189-527 + TS + Nup100p 571-959 \\
\hline pMR162 & Nup100-GLFG Nup100anchor & Nup100p $1+$ GS + Nup100p 2-580 + TS + Nup100p 571-959 \\
\hline pMR163 & Nup116-GLFG Nup100anchor & Nup100p $1+$ GS + Nup116p 2-725 + TS + Nup100p 571-959 \\
\hline pMR164 & Nup42-FG Nup100anchor & Nup100p $1+$ GS + Nup42p 4-364 + AS + Nup100p 571-959 \\
\hline pMR165 & Nup60-FG Nup100anchor & $\begin{array}{l}\text { Nup100p } 1+\text { GS + Nup60p 397-512 + AS + Nup100p 571- } \\
959\end{array}$ \\
\hline pMR166 & Nup145N-GLFG Nup100anchor & Nup100p $1+$ GS + Nup145 10-209 + AS + Nup100p 571-959 \\
\hline pMR167 & Nup159-FG Nup100anchor & $\begin{array}{l}\text { Nup100p } 1+\text { GS + Nup159p 464-876 + AS + Nup100p 571- } \\
959\end{array}$ \\
\hline pMR195 & Nup49-GLFG Nup49anchor & MFGS + Nup49p 2-236 + TS + Nup49p 237-472 \\
\hline pMR202 & Nup100-GLFG Nup49anchor & MFGS + Nup100p 2-580 + TS + Nup49p 237-472 \\
\hline pMR216 & Nup49-GLFG Nup57anchor & MNGS + Nup49p 2-236 + TS + Nup57p 224-541 \\
\hline pMR217 & Nup57-GLFG Nup57anchor & MNGS + Nup57p 2-223 + AS + Nup57p 224-541 \\
\hline pMR218 & Nsp1-FG Nup57anchor & MNGS + Nsp1p 2-602 + TS + Nup57p 224-541 \\
\hline pMR223 & Nup100-GLFG Nup57anchor & MNGS + Nup100p 2-580 + TS + Nup57p 224-541 \\
\hline pMR224 & Nup116-GLFG Nup57anchor & MNGS + Nup116p 2-725 + AS + Nup57p 224-541 \\
\hline pMR227 & Nup145N-GLFG Nup57anchor & MNGS + Nup145Np 10-209 + AS + Nup57p 224-541 \\
\hline pMR279 & GLEBS-Nup116anchor & Nup116p 1-6 + 140-175 + GLRSTQT + Nup116p 715-1113 \\
\hline pMR283 & $\begin{array}{l}\text { Nsp1FG-GLEBS-FSFG- } \\
\text { Nsp1anchor }\end{array}$ & $\begin{array}{l}\text { Nsp1p 1-271 + KL + Nup116p 105-175 + GLR + Nsp1p 273- } \\
602+\mathrm{T}+603-823\end{array}$ \\
\hline pMR293 & clean Nsp1 anchor & Nsp1p 1-2+603-823 \\
\hline pMR294 & clean Nup49 anchor & Nup49p $1+237-472$ \\
\hline pMR295 & clean Nup57 anchor & Nup57p $1+224-541$ \\
\hline pMR299 & $\begin{array}{l}\text { Nup57GLFG-Nup49GLFG } \\
\text { Nsp1anchor }\end{array}$ & $\begin{array}{l}\text { Nsp1p 1-2+GS+ Nup57 2-233+FMG+ Nup49 2-236+T+ } \\
\text { Nsp1p 603-823 }\end{array}$ \\
\hline
\end{tabular}


Table 4.2 - Continued from previous page

\begin{tabular}{|c|c|c|}
\hline Plasmid & Insert & Coded protein sequence \\
\hline pMR300 & $\begin{array}{l}\text { Nup57GLFG-Nsp1FG- } \\
\text { Nup49GLFG Nsp1anchor }\end{array}$ & $\begin{array}{l}\text { Nsp1p 1-2+GS+Nup57 2-233+FMG+Nsp1p 2- } \\
601+\text { FMG+Nup49 2-236+T+Nsp1p 603-823 }\end{array}$ \\
\hline pMR306 & $\begin{array}{l}\text { Nup100FG-Nup49FG } \\
\text { Nsp1anchor }\end{array}$ & $\begin{array}{l}\text { Nsp1p 1-2+GS+Nup100p 2-580+T+Nup4923-236+T+Nsp1p } \\
603-823\end{array}$ \\
\hline pMR307 & $\begin{array}{l}\text { Nup116FG-Nup49FG } \\
\text { Nsp1anchor }\end{array}$ & $\begin{array}{l}\text { Nsp1p 1-2+GS+Nup116p 2-725+A+Nup49 23-236+T+Nsp1p } \\
603-823\end{array}$ \\
\hline pMR311 & Nup116anchor & Nup116p 1-6 + SAFTGGLT + Nup116p 715-1113 \\
\hline pMR313 & Nup49-GLFG Nup116anchor & Nup116p 1-6 + S + Nup49p 2-236 + T + Nup116p 715-1113 \\
\hline pMR314 & Nup57-GLFG Nup116anchor & Nup116p 1-6 + S + Nup57p 2-223 + A + Nup116p 715-1113 \\
\hline pMR315 & Nsp1-FG Nup116anchor & Nup116p 1-6 + S + Nsp1p 2-602 + T + Nup116p 715-1113 \\
\hline pMR316 & Nup100-GLFG Nup116anchor & Nup116p 1-6 + S + Nup100p 2-580 + T + Nup116p 715-1113 \\
\hline pMR317 & Nup145N-GLFG Nup116anchor & Nup116p 1-6 + S + Nup145 10-209 + A + Nup116p 715-1113 \\
\hline pMR336 & clean Nup116anchor & $M+N u p 116 p 715-1113$ \\
\hline pMR372 & 4xNup145FG Nsp1anchor & $\begin{array}{l}\text { MGSTS }+3 x(\text { Nup145Np 10-209 + AS })+\text { Nup145 10-209 + A } \\
+ \text { Nsp1p 603-823 }\end{array}$ \\
\hline pMR373 & 4xNup145FG Nup100anchor & MGSTS + 4x (Nup145Np 10-209 + AS) + Nup100p 571-959 \\
\hline pMR374 & 4xNup145FG Nup116anchor & $\begin{array}{l}\text { Nup116p } 1-6+\text { STS }+3 x(\text { Nup145Np } 10-209+\text { AS })+\text { Nup145 } \\
10-209+\text { A }+ \text { Nup116p } 715-1113\end{array}$ \\
\hline pMR398 & Nup100FG-Nterm-short1 & MGS + Nup100p 2-107 + TS + Nup100p 571-959 \\
\hline pMR399 & Nup100FG-Nterm-short2 & MGS + Nup100p 2-199 + S + Nup100p 571-959 \\
\hline pMR400 & Nup100FG-Nterm-short3 & MGS + Nup100p 2-296 + TS + Nup100p 571-959 \\
\hline pMR401 & Nup100FG-Nterm-short4 & MGS + Nup100p 2-403 + S + Nup100p 571-959 \\
\hline pMR402 & Nup100FG-Nterm-short5 & MGS + Nup100p 2-501 + Nup100p 571-959 \\
\hline pMR403 & Nup100FG-Cterm-short1 & $\mathrm{M}+\mathrm{Nup} 100 \mathrm{p} 107-580+\mathrm{TS}+\mathrm{Nup100p} 571-959$ \\
\hline pMR404 & Nup100FG-Cterm-short2 & MGS + Nup100p 200-580 + TS + Nup100p 571-959 \\
\hline pMR405 & Nup100FG-Cterm-short3 & $\mathrm{MG}+$ Nup100p 297-580 + TS + Nup100p 571-959 \\
\hline pMR406 & Nup100FG-Cterm-short4 & $\mathrm{M}+\mathrm{Nup} 100 \mathrm{p} 404-580+\mathrm{TS}+\mathrm{Nup} 100 \mathrm{p} 571-959$ \\
\hline pMR407 & Nup100FG-Cterm-short5 & MG + Nup100p 501-580 + TS + Nup100p 571-959 \\
\hline pMR408 & Nup1FxFG Nup116anchor & Nup116p 1-6 + S + Nup1p 384-888 + T + Nup116p 715-1113 \\
\hline pMR409 & Nup2FxFG Nup116anchor & Nup116p 1-6 + S + Nup2p 189-527 + T + Nup116p 715-1113 \\
\hline pMR410 & Nup42FG Nup116anchor & Nup116p 1-6 + S + Nup42p 4-364 + A + Nup116p 715-1113 \\
\hline pMR411 & Nup159FG Nup116anchor & $\begin{array}{l}\text { Nup116p 1-6 + S + Nup159p 464-876 + A + Nup116p 715- } \\
1113\end{array}$ \\
\hline pMR412 & Nup60FG Nup116anchor & $\begin{array}{l}\text { Nup116p 1-6 + S + Nup60p 397-512 + A + Nup116p 715- } \\
1113\end{array}$ \\
\hline pSF1191 & Nup116 $\Delta$ GLEBS & Nup116p 1-109 + 167-1113 \\
\hline pSF1196 & $\begin{array}{l}\text { NUP116 } \Delta \text { GLFG174-715- } \\
\text { Spacer }\end{array}$ & $\begin{array}{l}\text { Nup116p 1-175 + 16x(NSGSGTGSSG })+\mathrm{T}+\text { Nup116p 715- } \\
1113\end{array}$ \\
\hline
\end{tabular}

Table 4.3: Additional plasmids used in this study Plasmids listed in this table originate from pRS415 (CEN LEU2 Amp).

\begin{tabular}{llcccc}
\hline Plasmid & Insert & Promotor & Terminator & Backbone & Marker \\
\hline pMR061 & NSP1 & $A D H 1$ & NUF2 & pRS316 & URA3 Amp \\
pMR062 & NUP49 & $A D H 1$ & NUF2 & pRS316 & URA3 Amp \\
pMR063 & NUP57 & ADH1 & NUF2 & pRS316 & URA3 Amp \\
\hline & & & & \multicolumn{2}{c}{ Continued on next page }
\end{tabular}




\begin{tabular}{|c|c|c|c|c|c|}
\hline Plasmid & Insert & Promotor & Terminator & Backbone & Marker \\
\hline pMR069 & NSP1 NUP49 NUP57 & \multicolumn{2}{|c|}{ endogenous } & $\mathrm{pRS} 416$ & URA3 $A m p$ \\
\hline pMR092 & $\begin{array}{l}\text { knockIn nup } 49 \Delta F G \\
(\Delta 2-236)\end{array}$ & \multicolumn{2}{|c|}{ NUP49 } & pBlueScript & $A m p$ \\
\hline pMR096 & $\begin{array}{l}\text { knockIn nup } 1 \Delta F G \\
(\Delta 382-888)\end{array}$ & \multicolumn{2}{|c|}{ NUP1 } & pBlueScript & $A m p$ \\
\hline pMR097 & $\begin{array}{l}\text { knockIn nup } 1 \Delta(\Delta 384- \\
1043)\end{array}$ & \multicolumn{2}{|c|}{ NUP1 } & pBlueScript & $A m p$ \\
\hline pMR098 & $\begin{array}{l}\text { knockIn nup2 } \Delta F G \\
(\Delta 189-556)\end{array}$ & \multicolumn{2}{|c|}{ NUP2 } & pBlueScript & $A m p$ \\
\hline pMR099 & $\begin{array}{l}\text { knockIn nup 42 } \Delta F G \\
(\Delta 4-364)\end{array}$ & \multicolumn{2}{|c|}{ NUP42 } & pBlueScript & $A m p$ \\
\hline pMR100 & $\begin{array}{l}\text { knockIn nup } 60 \Delta F G \\
(\Delta 397-512)\end{array}$ & \multicolumn{2}{|c|}{ NUP60 } & pBlueScript & $A m p$ \\
\hline pMR109 & $N S P 1$ & $A D H 1$ & NUF2 & CENmut & URA3 Amp \\
\hline pMR114 & NUP49 & $A D H 1$ & NUF2 & CENmut & URA3 $A m p$ \\
\hline pMR115 & NUP57 & $A D H 1$ & NUF2 & CENmut & URA3 Amp \\
\hline pMR116 & NUP145 & $A D H 1$ & NUF2 & CENmut & URA3 $A m p$ \\
\hline pMR117 & NUP116 & $A D H 1$ & NUF2 & CENmut & URA3 Amp \\
\hline pMR139 & $\begin{array}{l}\text { loopOut } n s p 1 \Delta F G(\Delta 3- \\
\text { 602) }\end{array}$ & \multicolumn{2}{|c|}{$N S P 1$} & $\mathrm{pRS} 402 \mathrm{mod}$ & URA3 ADE2 Amp \\
\hline pMR141 & $\begin{array}{l}\text { loopOut nup } 57 \Delta F G \\
\text { ( } \triangle 2 \text {-223) }\end{array}$ & \multicolumn{2}{|c|}{ NUP57 } & pRS402mod & URA3 ADE2 Amp \\
\hline pMR142 & $\begin{array}{l}\text { loopOut nup } 145 \Delta F G \\
(\Delta 10 \text {-209) }\end{array}$ & \multicolumn{2}{|c|}{$N U P 145$} & pRS402mod & URA3 ADE2 Amp \\
\hline pMR143 & $\begin{array}{l}\text { loopOut nup } 100 \Delta F G \\
(\Delta 2-576)\end{array}$ & \multicolumn{2}{|c|}{ NUP100 } & pRS402mod & URA3 ADE2 Amp \\
\hline pMR151 & NUP100 & $A D H 1$ & NUF2 & CENmut & URA3 Amp \\
\hline pMR288 & $\begin{array}{l}\text { loopOut nsp } 1 F G \text { - } \\
\text { GLEBS-FSFG- } \\
\text { nsp1anchor }\end{array}$ & \multicolumn{2}{|c|}{$N S P 1$} & $\mathrm{pRS} 402 \mathrm{mod}$ & URA3 ADE2 Amp \\
\hline pMR326 & $\begin{array}{l}\text { loopOut nup116 } \Delta F G \\
(\Delta 2-714)\end{array}$ & \multicolumn{2}{|c|}{ NUP116 } & pRS402mod & URA3 ADE2 Amp \\
\hline pSF0234 & $n s p 1 \Delta N(\Delta 2-605)$ & $A D H 1$ & NUF2 & pRS316 & URA3 Amp \\
\hline pSF1126 & $N U P 145$ & $A D H 1$ & NUF2 & $\mathrm{pRS} 416$ & URA3 Amp \\
\hline pSF1127 & NUP116 & $A D H 1$ & NUF2 & pRS416 & URA3 $A m p$ \\
\hline pSF1128 & NUP159 & $A D H 1$ & NUF2 & pRS416 & URA3 Amp \\
\hline
\end{tabular}

\subsection{Methods}

\subsubsection{Standard Methods in Molecular Biology}

All standard methods in Molecular Biology were performed on the basis of Sambrook and Russell (2001) with modifications as described in this section. 


\subsubsection{Polymerase Chain Reaction (PCR)}

Specific DNA fragments can be amplified from template DNA by polymerase chain reaction (PCR)(Mullis et al., 1986). Use of synthesized primers enables the introduction of endonuclease restriction sites at both ends of the generated DNA fragment for subsequent cloning steps. Oligonucleotide synthesis of primers was performed by Sigma-Aldrich (desalted oligonucleotides were used, if not stated otherwise).

In general, a $100 \mu \mathrm{L}$ reaction mixture for PCR was composed as described below:

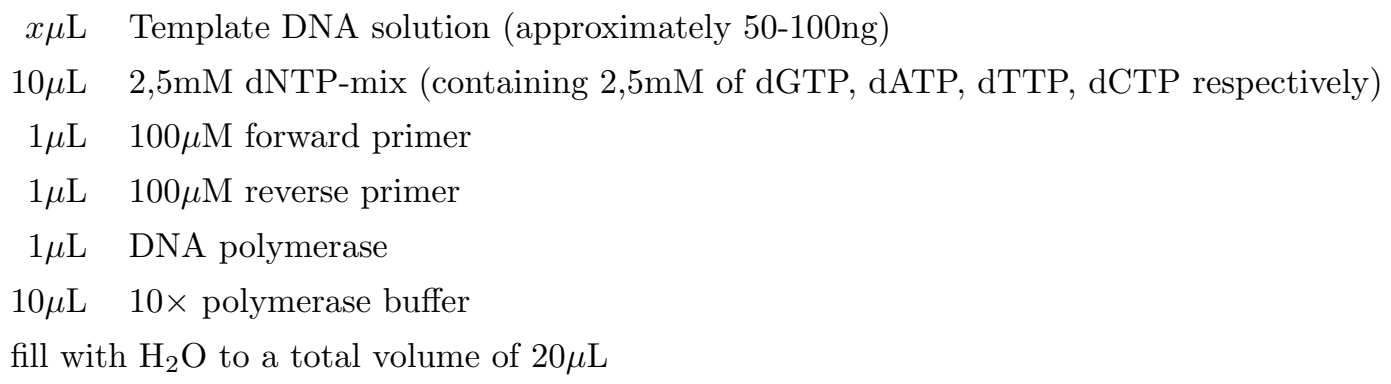

The polymerase and its buffer were chosen according to requirements: PfuS polymerase tripple mix (100ng/ $\mu \mathrm{L}$ PfuS DNA polymerase, $15 \mathrm{ng} / \mu \mathrm{L}$ Pab pyrophosphatase, $2,5 \mathrm{ng} / \mu \mathrm{L}$ Pab dUTPase; produced in the department by S. Frey) was used for general cloning, whereas Tth polymerase mix (100ng/ $\mu \mathrm{L}$ Tth DNA polymerase, $15 \mathrm{ng} / \mu \mathrm{L}$ Pab pyrophosphatase; produced in the department by S. Frey) was used for colony PCR reactions (see 4.2.1.7 or 4.2 .2 .7$)$.

PCR reactions were performed in a SensoQuest lab cycler, programmed with needed temperatures and durations, outlined here for a typical reaction with PfuS polymerase:

\section{Initial denaturation step}

$98^{\circ} \mathrm{C} \quad 2 \mathrm{~min}$

Amplification cycles (30-35 cycles)

$98^{\circ} \mathrm{C} \quad 30 \mathrm{sec}$

$t_{\text {annealing }} \quad 30 \mathrm{sec}$

$72^{\circ} \mathrm{C} \quad t_{\text {amplification }}$

Completion step

$72^{\circ} \mathrm{C} \quad 5 \mathrm{~min}$

$10^{\circ} \mathrm{C} \quad \infty$

$t_{\text {annealing }}$ stands for the average annealing temperature of a specific primer pair calculated by Oligo software and $t_{\text {amplification }}$ represents the duration of amplification, depending on the expected sequence length and amplification rate of the DNA polymerase (estimated to 
be $\sim 30 \mathrm{sec} / \mathrm{kb}$ for PfuS- and $\sim 40-60 \mathrm{sec} / \mathrm{kb}$ for Tth DNA Polymerase). In reactions were Tth DNA polymerase was used, denaturing temperature was lowered to $94^{\circ} \mathrm{C}$.

\subsubsection{Mutagenesis Polymerase Chain Reaction}

Mutagenesis PCR allows (i) to delete a small part of a gene, (ii) to exchange single amino acids coded in a gene, or (iii) to introduce base pairs coding for additional amino acids. For this purpose PCR primers were designed that amplify the entire plasmid of interest. The changes originate from non-complementary base pairs of the primers. Apart from that the primers were complementary to the regions to be modified. For mutagenesis PCR HPLC-purified and phosphorylated primers were ordered. A PCR reaction with PfuS DNA polymerase was performed as described in 4.2.1.1. After the reaction, the PCR product was precipitated by addition of one volume isopropanol to the PCR reaction mixture followed by $10 \mathrm{~min}$ of centrifugation (table-top centrifuge, 13000rpm) at room temperature. The supernatant was removed and the resulting pellet was resuspended in $30 \mu \mathrm{L}$ digestion mix $\left(2510 \mu \mathrm{L} \mathrm{H}_{2} \mathrm{O}, 3 \mu \mathrm{L}\right.$ NEB buffer $\left.4,2 \mu \mathrm{L} \mathrm{DpnI}(20 \mathrm{U} / \mu \mathrm{L})\right)$ for digestion of the methylated template plasmid. The digestion reaction was incubated for $1-2 \mathrm{~h}$ at $37^{\circ} \mathrm{C}$. Subsequently, the mix was purified by DNA gel purification (4.2.1.4) and ligated overnight at $16^{\circ} \mathrm{C}(4.2 .1 .5) .1-2 \mu \mathrm{L}$ of the ligated PCR product reaction was transformed into E. coli (4.2.1.6) and plated on selective medium.

\subsubsection{DNA Gel Electrophoresis}

Corresponding to their size, double stranded DNA fragments can be separated by their migration behavior in an agarose matrix exposed to an electric field. This method was used to separate, analyse and purify DNA fragments created by PCR or restriction digestion. Depending on the size of the expected DNA fragments, $0,8 \%-3 \%(\mathrm{w} / \mathrm{v})$ of agarose was dissolved in TAE buffer by heating. For visualization of DNA, the intercalating dye ethidium bromide (EtBr) was added to a final concentration of $\sim 0,5 \mu \mathrm{g} / \mathrm{mL}$. The resulting gel solution was poured into custom-made gel slides. After solidification, the gel was transferred into a custom-made electrophoresis chamber and a DNA marker of known size (1kb DNA ladder, Fermentas) as well as DNA samples mixed with 1:10 (v/v) Orange G DNA loading buffer were loaded to the gel. Electrophoresis was carried out at $\sim 160 \mathrm{~V}$ for approximately 30min. DNA was detected by direct examination of the gel in UV light with Intas Gel Documentation System (Intas Science Imaging Instruments GmbH, Göttingen, Germany). 


\subsubsection{Purification of DNA fragments from Agarose Gel and Concentration Determination}

After DNA gel electrophoresis, separated DNA fragments were cut out from the gel and purified with the Zymoclean Gel DNA recovery kit (Zymo Research, CA, USA) according to manufacturer's instructions.

DNA concentrations were determined with a NanoDrop ND-2000 spectrophotometer.

\subsubsection{Enzymatic Restriction Digestion \& Ligation of DNA}

All restriction endonucleases were purchased from NEB (New England Biolabs, Beverly, MA, USA). Digestion reactions were carried out according to manufacturer's instructions and at least 2 -fold excess of enzyme was used for $1 \mathrm{~h}$ digestions at $37^{\circ} \mathrm{C}$. For over night digestion at $37^{\circ} \mathrm{C}$ the amount of restriction endonucleases was decreased.

Ligations were performed using the T4 ligase $(100 \mathrm{ng} / \mu \mathrm{L}$; produced in the department by S. Frey). Ligation reaction mix was set up as follows:

$$
\begin{array}{rl}
x \mu \mathrm{L} & \sim 30 \mathrm{fmol} \text { Vector DNA }(\sim 20 \mathrm{ng} / \mathrm{kb}) \\
x \mu \mathrm{L} & \sim 60 \text { fmol Insert DNA }(\sim 40 \mathrm{ng} / \mathrm{kb}) \\
1 \mu \mathrm{L} & \quad \mathrm{T} 4 \text { DNA ligase } \\
10 \mu \mathrm{L} & 10 \times \text { ligation buffer } \\
\text { fill with } \mathrm{H}_{2} \mathrm{O} \text { to a total volume of } 10 \mu \mathrm{L}
\end{array}
$$

To reduce religation reactions of the vector, DNA was dephosphorylated for 30min at $37^{\circ} \mathrm{C}$ with $1 \mu \mathrm{L}$ FastAP (Fermentas, Glen Burnie, Maryland, USA) prior to ligation. As a negative control and to evaluate the relative efficiency of a ligation reaction, digested and dephosphorylated vector was incubated without addition of inserts.

The ligation reaction mix was mixed, shortly spined down in a table-top centrifuge and incubated for $30 \mathrm{~min}$ at $37^{\circ} \mathrm{C}$ for sticky-end ligations or overnight at $16^{\circ} \mathrm{C}$ for blunt-end ligations. Afterwards $1 \mu \mathrm{L}$ of ligation reaction mix was introduced into Escherichia coli cells by electroporation (4.2.1.6).

\subsubsection{Transformation of Escherichia coli with DNA}

For electroporation, $45 \mu \mathrm{L}$ electro-competent Escherichia coli cell solution (prepared in the department by G. Kopp) was mixed in an electroporation cuvette (BioRad, Burlington, USA) with $1 \mu \mathrm{L}$ of DNA ligation reaction mix or $\sim 50 \mathrm{ng}$ of plasmid DNA. Electroporation 
was performed in a GenePulser ${ }^{T M}$ device (BioRad, Burlington, USA) according to manufacturer's instructions. Afterwards cells were recovered in $1 \mathrm{~mL} 2 \mathrm{YT}$ medium supplemented with $0,2 \%$ glucose without antibiotics and incubated for $45 \mathrm{~min}$ at $37^{\circ} \mathrm{C}$ in a Thermomixer comfort (Eppendorf, Germany) agitating with 1300rpm. Cells were subsequently plated on selective agar plates containing an appropriate antibiotic $(100 \mu \mathrm{g} / \mathrm{mL}$ Ampicillin or $25 \mu \mathrm{g} / \mathrm{mL}$ Kanamycin) and incubated overnight at $37^{\circ} \mathrm{C}$.

\subsubsection{Colony PCR with Escherichia coli cells}

A variation of the PCR method (4.2.1.1) was used to screen for correct cloning constructs after transformation of Escherichia coli cells. Instead of using purified DNA, living cells were added as a template. For this purpose a colony was picked and thoroughly resuspended in $50 \mu \mathrm{L}$ of medium. $1 \mu \mathrm{L}$ of the resulting cell suspension was added to $19 \mu \mathrm{L}$ PCR reaction mixture (see 4.2.1.1). Tth polymerase mix (100ng/ $\mu \mathrm{L}$ Tth DNA polymerase, $15 \mathrm{ng} / \mu \mathrm{L}$ Pab pyrophosphatase; produced in the department by S. Frey) was used for colony-PCR. The PCR reaction was followed by analysis via agarose gel electrophoresis (4.2.1.3). In case of a positive result, the remaining cell suspension was used to inoculate a culture for DNA purification.

\subsubsection{Plasmid DNA Purification from Escherichia coli}

Plasmid DNA from Escherichia coli was purified with the assistance of G. Kopp at analytical scale using the NucleoSpin ${ }^{\circledR}$ Plasmid Mini kit (Macherey Nagel, Germany) or at preparative scale using NucleoBond ${ }^{\circledR}$ Xtra Midi kit (Macherey Nagel, Germany) according to the manufacturer's instructions.

$2 \mathrm{~mL}$ of $2 \mathrm{YT}$ medium containing appropriate antibiotics were inoculated for analytical purification while $250 \mathrm{~mL}$ of $2 \mathrm{YT}$ medium were used for preparative purification. The cultures were incubated over night at $37^{\circ} \mathrm{C}$.

\subsubsection{DNA Sequencing}

DNA sequencing reactions of plasmids or purified DNA fragments generated by PCR were performed by Seqlab-Sequence Laboratories Göttingen GmbH. The results were analyzed with SeqMan Software.

\subsubsection{Methods in Saccharomyces cerevisiae Genetics}




\subsubsection{General Culture and Storage of Saccharomyces cerevisiae}

Strains were cultured at $30^{\circ} \mathrm{C}$ or, if necessary, at $25^{\circ} \mathrm{C}$ either in YPD medium or in SDmedium, selecting for the appropriate auxotrophy marker.

Growth of liquid cultures was monitored by measuring the optical density at $600 \mathrm{~nm}$ $\left(\mathrm{OD}_{600}\right)$ with a Eppendorf BioPhotometer plus.

For long-term storage, glycerol culture stocks were prepared. To do so, $1 \mathrm{~mL}$ of a culture grown to stationary phase was mixed with $800 \mu \mathrm{L}$ of sterile glycerol $(86 \%(\mathrm{v} / \mathrm{v}))$ and directly stored at $-80^{\circ} \mathrm{C}$.

\subsubsection{Transformation of Saccharomyces cerevisiae with DNA}

The transformation procedure was based on a protocol developed by Gietz et al. (1995) and was applied as follows: A $10 \mathrm{~mL}$ pre-culture of the strain to be transformed was grown overnight $(\sim 16 \mathrm{~h})$ or for up to $40 \mathrm{~h}$ to stationary phase $\left(\mathrm{OD}_{600} \gtrsim 2\right)$. It was then used to inoculate a $25 \mathrm{~mL}$ culture to an $\mathrm{OD}_{600}$ of $\sim 0,1$. This main culture was incubated until grown to an $\mathrm{OD}_{600}$ between 0,5 and 0,8 . Cells were harvested by centrifugation $(5 \mathrm{~min}$, room temperature, 4500rpm, Swing-out rotor 75006445 in Thermo Scientific Multifuge 3L$\mathrm{R}$ ), washed once in $20 \mathrm{~mL}$ sterile $\mathrm{H}_{2} \mathrm{O}$, once in $10 \mathrm{~mL} \mathrm{LiAc/TE}$-solution, and resuspended in $100 \mu \mathrm{L} \mathrm{LiAc/TE}$-solution. $1 \mu \mathrm{g}$ of transformation DNA, $8 \mu \mathrm{L}$ denatured salmon sperm DNA solution $(\sim 10 \mathrm{mg} / \mathrm{mL})$, and $500 \mu \mathrm{L}$ PEG/LiAc/TE-solution were added to the cells and mixed by vortexing thoroughly. Cells were incubated for at least $1 \mathrm{~h}$ at $25^{\circ} \mathrm{C}$ (up to $3 \mathrm{~h}$ ) and heat-shocked for $20 \mathrm{~min}$ at $42^{\circ} \mathrm{C}$.

$200 \mu \mathrm{L}$ of this mix were plated on selection agar plates and incubated at $25^{\circ} \mathrm{C}$. When linear dsDNA was transformed, the remaining mix was shortly centrifuged and the PEG supernatant was removed. Pelleted cells were resuspended in $300 \mu \mathrm{L}$ sterile $\mathrm{H}_{2} \mathrm{O}$ and plated as well.

\subsubsection{Genomic Gene Deletion}

Genomic genes were deleted by transformation of cells with PCR-generated gene replacement cassettes (figure 4.1A). A gene replacement cassette for gene deletion consisted of two marker genes (HIS3 or URA3 in addition to ADE2) that were flanked by sequences homologous to the 5'-UTR and 3'-UTR of the target gene, respectively. Employment of the ADE2 marker allowed for additional selection via red/white screening.

In case that the targeted gene was essential, a rescue vector had been transformed before 
deletion. To avoid targeting of a gene replacement cassette to the vector, the ADH1 promotor was chosen as a 5'-UTR for the rescue construct, whereas the 3'-UTR of the gene was replaced by the NUF2 3'-UTR. See table 4.4 for a summary of gene deletion cassettes used in this study.

To verify genomic gene deletion, the genomic locus was analyzed by PCR with primers annealing up- and downstream of the region where homologous recombination occurred.

Table 4.4: Gene deletion cassettes generated by PCR

\begin{tabular}{llll}
\hline Deletion cassette & Template & Primer & Rescue vector \\
\hline NSP1::HIS3-ADE2 & pSF1198 & SF1110 \& SF1111 & pMR109 \\
NUP1::URA3-ADE2 & pSF1197 & SF1090 \& SF1091 & $/$ \\
NUP2::URA3-ADE2 & pSF1177 & SF1092 \& SF1093 & $/$ \\
NUP42::URA3-ADE2 & pSF1178 & SF1094 \& SF1095 & $/$ \\
NUP60::URA3-ADE2 & pSF1179 & SF1096 \& SF1097 & $/$ \\
NUP49::HIS3-ADE2 & pSF1200 & SF1098 \& SF1099 & pMR62 or pMR114 \\
NUP57::HIS3-ADE2 & pSF1201 & SF1100 \& SF1101 & pMR63 or pMR115 \\
NUP145::HIS3-ADE2 & pSF1202 & SF1102 \& SF1103 & pSF1126 or pMR116 \\
NUP100::URA3-ADE2 & pSF1180 & SF1104 \& SF1105 & $/$ \\
NUP100::HIS3-ADE2 & pMR152 & SF1104 \& SF1105 & pMR151 \\
NUP116::HIS3-ADE2 & pSF1203 & SF1106 \& SF1107 & pSF1127 or pMR117 \\
NUP159::HIS3-ADE2 & pSF1204 & SF1108 \& SF1109 & pSF1128 or pMR118 \\
\hline
\end{tabular}

(A)

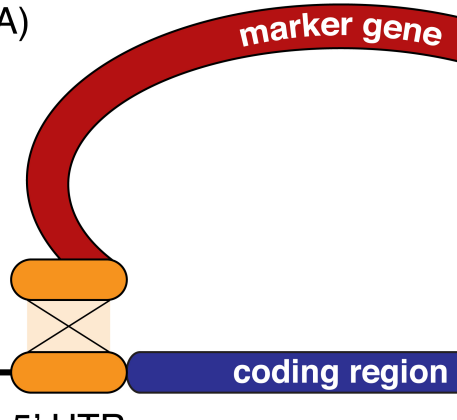

5' UTR

Figure 4.1: Principle of gene deletion and gene insertion via replacement. (A) A selectable marker gene is targeted to the gene of interest by flanking regions, which are homologous to the 5'-UTR and 3'UTR of the coding region of the target gene. The marker gene is then integrated by homologous recombination and replaces the coding region of the gene of interest. (B) A similar strategy can be applied to integrate a gene coding a $\triangle F G$ nucleoporin mutant. To do so, the coding sequence is previously deleted by replacement with the URA3 marker gene. Insertion of the $\triangle \mathrm{FG}$ mutant coding gene via homologous recombination results then in cells that are no longer sensitive to FOA (see 4.2.2.9 for details of resistance). 


\subsubsection{Genomic Deletion of FG Domains}

Two strategies were applied to genomically delete FG repeat domains of nucleoporins. First, as depicted in figure $4.1 \mathrm{~B}$, a strategy similar to that used for genomic gene deletions was pursued. For this purpose, the nucleoporin gene was previously disrupted by a gene replacement cassette containing the URA3 marker gene. In case of an essential nucleoporin, the replacement cassette contained the HIS3 marker gene and the according rescue vector coded the URA3 marker. Such a strain was then transformed with a second gene replacement cassette (see table 4.5). This cassette contained a gene, which coded for a $\triangle F G$ form of the nucleoporin and was flanked by the endogenous UTRs responsible for targeting the cassette to the genomic locus. Replacement cassettes were generated by restriction digestion of the appropriate plasmid. After transformation, cells were incubated in YPD medium over night before counter selection on FOA plates was performed.

Resulting colonies were screened by colony PCR. To further verify correct insertion of the gene replacement cassette and to rule out any unwanted mutation, the genomic locus was amplified by PCR with primers annealing up- and downstream of the regions of homologous recombination and the resulting PCR product was sequenced.

For unknown reasons, this strategy was only successful for some nucleoporin genes. Therefore a second strategy was applied: The genes coding for $\Delta \mathrm{FG}$ forms of the nucleoporins including their flanking region were cloned into yeast integration vectors (pRS400 series by Sikorski and Hieter, 1989; Christianson et al., 1992) containing the URA3 and the ADE2 marker gene. The resulting vectors were linearized by restriction digestion with an endonuclease recognizing a unique target site within the coding region of the nucleoporin gene (see table 4.5).

A vector prepared in this way was then transformed and cells that integrated the vector were selected via plating on CSM-Ura. A successful integration event, i.e. a "loop-in" event, resulted in two copies of the nucleoporin gene at the genomic locus: a wild type and a $\triangle$ FG coding allele (figure $4.2 \mathrm{~B}$, page 82 ), respectively. To allow the occurrence of a "loop-out" event (figure $4.2 \mathrm{C}$ ), a colony was grown for two days in $\sim 5 \mathrm{~mL}$ YPD liquid medium. $5-20 \mu \mathrm{L}$ of this culture were plated on FOA plates and incubated until colonies were visible. Since the "loop-out" event could result in either a wild type or a $\Delta$ FG coding version of the gene being present at the locus, resulting colonies were screened by colony $\mathrm{PCR}$. To verify the exclusive presence of the $\triangle \mathrm{FG}$ allele, genomic DNA was prepared and the genomic locus was amplified by PCR with primers annealing up- and downstream of 
the recombination regions. The resulting PCR product was sequenced in order to rule out any unwanted mutation of the coding sequence.

Table 4.5: Constructs for the insertion of mutated nucleoporin genes

\begin{tabular}{|c|c|c|c|}
\hline Construct & Strategy & Plasmid & $\begin{array}{l}\text { Restriction } \\
\text { with }\end{array}$ \\
\hline nup $49 \Delta F G(\Delta 2-236)$ & Gene insertion via replacement & pMR92 & NotI \& XhoI \\
\hline nup $1 \Delta F G(\Delta 382-888)$ & Gene insertion via replacement & pMR96 & $\begin{array}{l}\text { PvuI \& PvuII } \\
\& \text { EcoRV }\end{array}$ \\
\hline nup $1 \Delta F G(\Delta 384-1043)$ & Gene insertion via replacement & pMR97 & $\begin{array}{l}\text { PvuI \& PvuII } \\
\& \text { EcoRV }\end{array}$ \\
\hline nup2 $\Delta F G(\Delta 189-556)$ & Gene insertion via replacement & pMR98 & NspI \& XhoI \\
\hline nup42 $\Delta F G(\Delta 4-364)$ & Gene insertion via replacement & pMR99 & $A c c \mathrm{I} \& A h d \mathrm{I}$ \\
\hline nup60 $\Delta F G(\Delta 397-512)$ & Gene insertion via replacement & pMR100 & AccI \& EcoRI \\
\hline$n s p 1 \Delta F G(\Delta 3-602)$ & Gene domain deletion via loop-in loop-out & pMR139 & SnaBI \\
\hline nup $57 \Delta F G(\Delta 2-223)$ & Gene domain deletion via loop-in loop-out & pMR141 & BsiWI \\
\hline nup 145 $\Delta F G(\Delta 10-209)$ & Gene domain deletion via loop-in loop-out & pMR142 & SnaBI \\
\hline nup $100 \Delta F G(\Delta 2-576)$ & Gene domain deletion via loop-in loop-out & pMR143 & $B m g \mathrm{BI}$ \\
\hline nup116 $\Delta F G(\Delta 2-714)$ & Gene domain deletion via loop-in loop-out & pMR326 & EcoRI \\
\hline$N S P 1-G L E B S$-fusion construct ${ }^{\mathbf{a}}$ & Gene domain deletion via loop-in loop-out & pMR288 & $S f o \mathrm{I}$ \\
\hline \multicolumn{4}{|c|}{$\begin{array}{l}\text { a The GLEBS domain of Nup116p (amino acids 105-175) (Bailer et al., 1998) was inserted into the FG domain } \\
\text { of Nsp1p between amino acids } 271 \text { and } 273 \text {. The construct codes for the following exact amino acid sequence: } \\
\text { Nsp1p 1-271 + KL + Nup116p 105-175 + GLR }+ \text { Nsp1p 273-602 + T + Nsp1p 603-823. }\end{array}$} \\
\hline
\end{tabular}

\subsubsection{Preparation of Genomic DNA from Saccharomyces cerevisiae}

Yeast genomic DNA was prepared using the YeaStar ${ }^{\mathrm{TM}}$ Genomic DNA kit (Zymo Research, CA, USA) according to the manufacturer's instructions. DNA was eluted in $70 \mu \mathrm{L} \mathrm{H}_{2} \mathrm{O}$.

\subsubsection{Preparation of Plasmid DNA from Saccharomyces cerevisiae}

A modified protocol of Singh and Weil (2002) was employed to isolate plasmid DNA from Saccharomyces cerevisiae as described below. Solutions and columns of the NucleoSpin ${ }^{\circledR}$ Plasmid Mini kit (Macherey Nagel, Germany) were used.

Cells from a $10 \mathrm{~mL}$ overnight culture were collected by centrifugation and resuspended in $250 \mu \mathrm{L}$ solution A1. To digest the cell wall, $5 \mu \mathrm{L}$ R-Zymolyase ${ }^{\top \mathrm{M}}(1000 \mathrm{U} / \mathrm{mL}$; Zymo Research, CA, USA) was added. The mix was thoroughly vortexed and incubated for at least $45 \mathrm{~min}$ (overnight incubation is possible as well) at $37^{\circ} \mathrm{C}$. Cell lysis was then accomplished by addition of $250 \mu \mathrm{L}$ solution A2 followed by a gentle inversion of the reaction tube and incubation at room temperature. After $10 \mathrm{~min} 300 \mu \mathrm{L}$ solution A3 were added and the mix was centrifuged (10min, room temperature, 14.000rpm, Eppendorf table top centrifuge). 


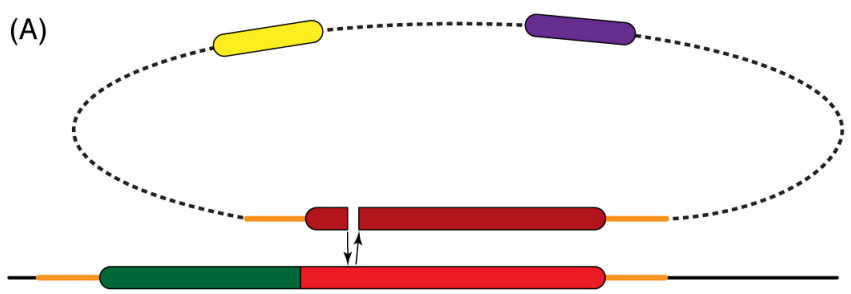

(B)

(C)

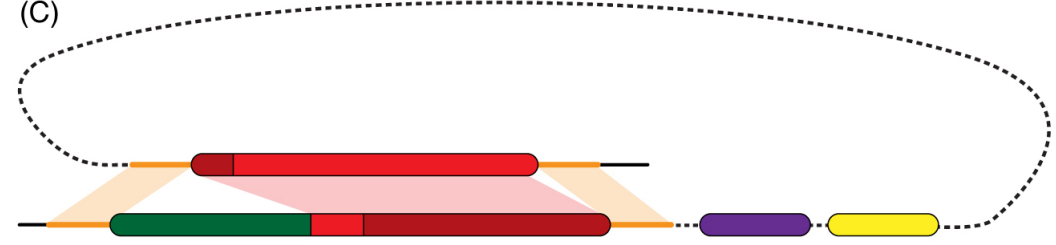

(D)

(E)

Figure 4.2: Principle of gene domain deletion via loop-in loop-out. (A) Yeast integration vectors are linearized within the coding region of the nucleoporin (red) lacking the FG domain (shown in green for the genomic gene). Non-coding homologous regions are depicted in orange. After transformation, the linearized vector can integrate into the genomic locus as shown in (B) resulting in two copies of the nucleoporin gene (with and without the FG domain, respectively). These are separated by the sequence originating from the yeast integration vector (dashed line, marker genes in yellow and purple, respectively). (C) Due to sequence homology, a loop-out event can occur and recombination can eventuate at any part of the homologous regions, so that the loop-out event can have two different overall results: (D) either recombination occurs in the 5'-UTR (orange) finally resulting in the deletion of the FG domain coding sequence or (E) a wild type situation is restored. 
The supernatant was applied to a column via a short centrifugation step. The column was afterwards washed twice with $500 \mu \mathrm{L}$ prewarmed $\left(\sim 50^{\circ} \mathrm{C}\right)$ solution $\mathrm{AW}$ and $600 \mu \mathrm{L}$ solution A4 respectively. $50 \mu \mathrm{L} \mathrm{H}_{2} \mathrm{O}$ were added and DNA was eluted after 1min incubation at room temperature by centrifugation for 30sec.

\subsubsection{Colony PCR with Saccharomyces cerevisiae cells}

Screening of positive clones after transformation of DNA was facilitated by colony PCR, which avoids the need of DNA purification for characterisation. As distinguished from standard PCR (4.2.1.1) whole cells were used as PCR template. Colonies to be tested were picked from a agar plate and resuspended in $25 \mu \mathrm{L}$ digestion buffer containing $50 \mathrm{mM}$ Tris $(\mathrm{pH} 8,5)$ and 50U/mL R-Zymolyase ${ }^{\mathrm{TM}}$ (Zymo Research, CA, USA). Directly after resuspending the cells, $1 \mu \mathrm{L}$ of the suspension was spotted on fresh agar medium to allow cell growth of the potentially positive clones. The remaining suspension was incubated overnight in an Eppendorf Thermomixer plus at $37^{\circ} \mathrm{C}$ with $1300 \mathrm{rpm}$ agitation to digest cell walls.

The next day the thermomixer was heated to $95^{\circ} \mathrm{C}$ for $10 \mathrm{~min}$ to denature proteins and thereby lyse the cells. To collect condensed water from the lid of the reaction tubes, the tubes were briefly centrifuged before $1 \mu \mathrm{L}$ of the supernatant was used as a template for the PCR reaction with Tth DNA Polymerase (see 4.2.1.1 for PCR mix and conditions).

\subsubsection{Sporulation \& Tetrad Dissection}

Diploid strains were sporulated by growing them first for three days on YPD plates and for further 10 to 16 days on sporulation plates at $25^{\circ} \mathrm{C}$. Sporulation was monitored by lightmicroscopic observation with an Olympus BX50 (Kassir and Simchen, 1991). To detach the wall of the ascus from the spores, tetrads were scratched from the sporulation plate with a sterile inoculating loop. After suspension in $500 \mu \mathrm{L} \mathrm{H}_{2} \mathrm{O}$ supplemented with $10 \mu \mathrm{L}$ $\beta$-D-glucuronidase/arylsulfatase from Helix pomatia (Roche Diagnostics, Mannheim, Germany), tetrads were briefly mixed and incubated for exactly $8 \mathrm{~min}$ at room temperature (Johnston and Mortimer, 1959). 5-10 $\mu \mathrm{L}$ of the suspension were streaked at the edge of a YPD plate. Segregation of the four spores of a tetrad was carried out using a micromanipulator (Singer MSM System Series 300). Dissected spores were allowed to grow for $\sim 7$ days at $25^{\circ} \mathrm{C}$ before growth behaviour on several selection media was observed. 


\subsubsection{FOA Complementation Assay}

This assay allows to monitor the complementation strength of a mutated allele versus the wild type allele of an essential gene.

Crucial for this assay is the cytotoxic effect of the pyrimidine analog 5-fluoro-orotic acid (FOA) in strains bearing a functional URA3 gene. The URA3 gene product orotidine5'-phosphate decarboxylase converts FOA into the toxic compound 5-fluorouracil (Boeke et al., 1984). For this reason wild type yeast cells or cells containing a plasmid coded URA3 gene are unable to grow on medium containing FOA.

To analyse the complementation strength of a mutated gene, a strain is used, in which an essential gene of interest is coded only by a plasmid containing the URA3 selection marker gene. This strain is transformed with a second plasmid coding for the mutated allele of the essential gene. If both alleles are functional, cells forfeit one of the two plasmids by a random process. When plated on FOA containing medium, only those cells are able to grow, in which the mutated allele of the gene can complement for the loss of the wild type gene.

The assay was performed as follows: A strain of interest containing a plasmid with the URA3 selection marker was grown in CSM-Ura medium for up to two days and was then transformed as described in 4.2.2.2 with a second plasmid containing the LEU2 selection marker. Transformants were selected on double selective plates (CSM-Ura-Leu) to avoid spontaneous mutations of the URA3 gene. To allow for loss of the URA3 coding plasmid, a single colony was picked, resuspended in $3 \mathrm{~mL}$ CSM-Leu medium, and incubated at $25^{\circ} \mathrm{C}$ until the culture reached stationary phase (in general after $\sim 40 \mathrm{~h}$ for strains with multiple FG repeat domain deletions).

Using this culture, cells were diluted in sterile $\mathrm{H}_{2} \mathrm{O}$ to yield $1 \mathrm{~mL}$ with an $\mathrm{OD}_{600}$ of 0,6 . Ten-fold dilution series of this $\mathrm{OD}_{600} 0,6$ culture were prepared in a 96 well plate (TPP, Trasadingen, Switzerland) and spotted on FOA containing plates, YPD plates, and CSMLeu plates with a multi channel pipette (Eppendorf research pro). The plates were incubated at $25^{\circ} \mathrm{C}$. Pictures of plates were acquired after two to 14 days by scanning with an Epson Perfection V700 Photo Scanner using "Epson Scan" Software (Version 3.28A) set to produce 8 bit grayscale images with 400dpi. 


\subsubsection{Temperature Sensitivity Assay}

To assay growth properties at a range of temperatures, strains were incubated in $3 \mathrm{~mL}$ YPD medium at $25^{\circ} \mathrm{C}$ until the culture reached stationary phase. Using this culture, cells were diluted in YPD to yield $1 \mathrm{~mL}$ with an $\mathrm{OD}_{600}$ of 0,6 . Ten-fold dilution series of this $\mathrm{OD}_{600}$ 0,6 culture were prepared in a 96 well plate (TPP, Trasadingen, Switzerland) and spotted on four YPD plates with a multi channel pipette (Eppendorf research pro). The plates were incubated at $16^{\circ} \mathrm{C}, 25^{\circ} \mathrm{C}, 30^{\circ} \mathrm{C}$, and $37^{\circ} \mathrm{C}$ respectively. Pictures of the plates were acquired after two to 14 days by scanning with an Epson Perfection V700 Photo Scanner using "Epson Scan" Software (Version 3.28A) set to produce 8bit grayscale images with 400dpi.

\subsubsection{Confocal Microscopy}

Proteins were genetically fused to Citrine (Griesbeck et al., 2001), an enhanced version of the yellow fluorescent protein, to study their intracellular localization. Cells expressing Citrine fusion proteins were grown to stationary phase and $5 \mu \mathrm{L}$ of the culture were directly loaded on Superfrost UltraPlus microscope slides (Menzel GmbH, Braunschweig, Germany). Confocal images were acquired with a Leica SP5 confocal laser scanning microscope equipped with a $20 \times$ or $63 \times$ immersion objective (Leica, Wetzlar, Germany). 


\section{Bibliography}

Ader, C., Frey, S., Maas, W., Schmidt, H. B., Görlich, D., and Baldus, M. (2010). Amyloid-like interactions within nucleoporin FG hydrogels. Proc Natl Acad Sci USA, 107(14):6281-5.

Aitchison, J. D., Blobel, G., and Rout, M. P. (1995a). Nup120p: a yeast nucleoporin required for NPC distribution and mRNA transport. J Cell Biol, 131(6 Pt 2):1659-75.

Aitchison, J. D. and Rout, M. P. (2012). The yeast nuclear pore complex and transport through it. Genetics, 190(3):855-83.

Aitchison, J. D., Rout, M. P., Marelli, M., Blobel, G., and Wozniak, R. W. (1995b). Two novel related yeast nucleoporins Nup170p and Nup157p: complementation with the vertebrate homologue Nup155p and functional interactions with the yeast nuclear pore-membrane protein Pom152p. J Cell Biol, 131(5):1133-48.

Akey, C. W. and Goldfarb, D. S. (1989). Protein import through the nuclear pore complex is a multistep process. J Cell Biol, 109(3):971-82.

Akey, C. W. and Radermacher, M. (1993). Architecture of the Xenopus nuclear pore complex revealed by three-dimensional cryo-electron microscopy. J Cell Biol, 122(1):1-19.

Alber, F., Dokudovskaya, S., Veenhoff, L. M., Zhang, W., Kipper, J., Devos, D., Suprapto, A., Karni-Schmidt, O., Williams, R., Chait, B. T., Sali, A., and Rout, M. P. (2007). The molecular architecture of the nuclear pore complex. Nature, 450(7170):695-701.

Bahr, G. and Beermann, W. (1954). The fine structure of the nuclear membrane in the larval salivary gland and midgut of Chironomus. Exp Cell Res, 6(2):519-22.

Baï, S. W., Rouquette, J., Umeda, M., Faigle, W., Loew, D., Sazer, S., and Doye, V. (2004). The fission yeast Nup107-120 complex functionally interacts with the small GTPase Ran/Spi1 and is required for mRNA export, nuclear pore distribution, and proper cell division. Mol Cell Biol, 24(14):6379-92.

Bailer, S. M., Balduf, C., and Hurt, E. (2001). The Nsp1p carboxy-terminal domain is organized into functionally distinct coiled-coil regions required for assembly of nucleoporin subcomplexes and nucleocytoplasmic transport. Mol Cell Biol, 21(23):7944-55.

Bailer, S. M., Balduf, C., Katahira, J., Podtelejnikov, A., Rollenhagen, C., Mann, M., Pante, N., and Hurt, E. (2000). Nup116p associates with the Nup82p-Nsp1p-Nup159p nucleoporin complex. J Biol Chem, 275(31):23540-8. 
Bailer, S. M., Siniossoglou, S., Podtelejnikov, A., Hellwig, A., Mann, M., and Hurt, E. (1998). Nup116p and Nup100p are interchangeable through a conserved motif which constitutes a docking site for the mRNA transport factor Gle2p. EMBO J, 17(4):1107-19.

Bayliss, R., Leung, S. W., Baker, R. P., Quimby, B. B., Corbett, A. H., and Stewart, M. (2002a). Structural basis for the interaction between NTF2 and nucleoporin FxFG repeats. EMBO J, 21(12):2843-53.

Bayliss, R., Littlewood, T., and Stewart, M. (2000). Structural basis for the interaction between FxFG nucleoporin repeats and importin-beta in nuclear trafficking. Cell, 102(1):99-108.

Bayliss, R., Littlewood, T., Strawn, L. A., Wente, S. R., and Stewart, M. (2002b). GLFG and FxFG nucleoporins bind to overlapping sites on importin-beta. J Biol Chem, 277(52):50597-606.

Bayliss, R., Ribbeck, K., Akin, D., Kent, H. M., Feldherr, C. M., Görlich, D., and Stewart, M. (1999). Interaction between NTF2 and xFxFG-containing nucleoporins is required to mediate nuclear import of RanGDP. Journal of Molecular Biology, 293(3):579-93.

Beck, M., Förster, F., Ecke, M., Plitzko, J. M., Melchior, F., Gerisch, G., Baumeister, W., and Medalia, O. (2004). Nuclear pore complex structure and dynamics revealed by cryoelectron tomography. Science (New York, NY), 306(5700):1387-90.

Beck, M., Lučić, V., Förster, F., Baumeister, W., and Medalia, O. (2007). Snapshots of nuclear pore complexes in action captured by cryo-electron tomography. Nature, 449(7162):611-615.

Bednenko, J., Cingolani, G., and Gerace, L. (2003). Importin beta contains a COOH-terminal nucleoporin binding region important for nuclear transport. J Cell Biol, 162(3):391-401.

Belanger, K. D., Kenna, M. A., Wei, S., and Davis, L. I. (1994). Genetic and physical interactions between Srp1p and nuclear pore complex proteins Nup1p and Nup2p. J Cell Biol, 126(3):619-30.

Belgareh, N., Snay-Hodge, C., Pasteau, F., Dagher, S., Cole, C. N., and Doye, V. (1998). Functional characterization of a Nup159p-containing nuclear pore subcomplex. Mol Biol Cell, 9(12):347592 .

Belov, G. A., Lidsky, P. V., Mikitas, O. V., Egger, D., Lukyanov, K. A., Bienz, K., and Agol, V. I. (2004). Bidirectional increase in permeability of nuclear envelope upon poliovirus infection and accompanying alterations of nuclear pores. $J$ Virol, 78(18):10166-77.

Ben-Efraim, I. and Gerace, L. (2001). Gradient of increasing affinity of importin beta for nucleoporins along the pathway of nuclear import. J Cell Biol, 152(2):411-7.

Boeke, J. D., LaCroute, F., and Fink, G. R. (1984). A positive selection for mutants lacking orotidine-5'-phosphate decarboxylase activity in yeast: 5-fluoro-orotic acid resistance. Mol Gen Genet, 197(2):345-6.

Brohawn, S. G., Leksa, N. C., Spear, E. D., Rajashankar, K. R., and Schwartz, T. U. (2008). Structural evidence for common ancestry of the nuclear pore complex and vesicle coats. Science (New York, NY), 322(5906):1369-73. 
Brohawn, S. G., Partridge, J. R., Whittle, J. R. R., and Schwartz, T. U. (2009). The nuclear pore complex has entered the atomic age. Structure, 17(9):1156-68.

Brown, J. A., Bharathi, A., Ghosh, A., Whalen, W., Fitzgerald, E., and Dhar, R. (1995). A mutation in the Schizosaccharomyces pombe rae1 gene causes defects in poly(A)+ RNA export and in the cytoskeleton. J Biol Chem, 270(13):7411-9.

Chadrin, A., Hess, B., Roman, M. S., Gatti, X., Lombard, B., Loew, D., Barral, Y., Palancade, B., and Doye, V. (2010). Pom33, a novel transmembrane nucleoporin required for proper nuclear pore complex distribution. J Cell Biol, 189(5):795-811.

Chial, H. J., Rout, M. P., Giddings, T. H., and Winey, M. (1998). Saccharomyces cerevisiae Ndc1p is a shared component of nuclear pore complexes and spindle pole bodies. J Cell Biol, 143(7):1789-800.

Christianson, T. W., Sikorski, R. S., Dante, M., Shero, J. H., and Hieter, P. (1992). Multifunctional yeast high-copy-number shuttle vectors. Gene, 110(1):119-22.

Cordes, V. C., Reidenbach, S., Rackwitz, H. R., and Franke, W. W. (1997). Identification of protein p270/Tpr as a constitutive component of the nuclear pore complex-attached intranuclear filaments. J Cell Biol, 136(3):515-29.

Cronshaw, J. M., Krutchinsky, A. N., Zhang, W., Chait, B. T., and Matunis, M. J. (2002). Proteomic analysis of the mammalian nuclear pore complex. J Cell Biol, 158(5):915-27.

D'Angelo, M. A. and Hetzer, M. W. (2008). Structure, dynamics and function of nuclear pore complexes. Trends in Cell Biology, 18(10):456-66.

Davis, L. I. and Blobel, G. (1987). Nuclear pore complex contains a family of glycoproteins that includes p62: glycosylation through a previously unidentified cellular pathway. Proc Natl Acad Sci USA, 84(21):7552-6.

Davis, L. I. and Fink, G. R. (1990). The NUP1 gene encodes an essential component of the yeast nuclear pore complex. Cell, 61(6):965-78.

de Castillia, C. S., Blobel, G., and Rout, M. P. (1999). Proteins connecting the nuclear pore complex with the nuclear interior. J Cell Biol, 144(5):839-55.

Denning, D., Mykytka, B., Allen, N. P., Huang, L., Burlingame, A., and Rexach, M. (2001). The nucleoporin Nup60p functions as a Gsp1p-GTP-sensitive tether for Nup2p at the nuclear pore complex. J Cell Biol, 154(5):937-50.

Denning, D. P., Patel, S. S., Uversky, V., Fink, A. L., and Rexach, M. (2003). Disorder in the nuclear pore complex: the FG repeat regions of nucleoporins are natively unfolded. Proc Natl Acad Sci USA, 100(5):2450-5.

Denning, D. P. and Rexach, M. F. (2007). Rapid evolution exposes the boundaries of domain structure and function in natively unfolded FG nucleoporins. Mol Cell Proteomics, 6(2):272-82. 
Denning, D. P., Uversky, V., Patel, S. S., Fink, A. L., and Rexach, M. (2002). The Saccharomyces cerevisiae nucleoporin Nup2p is a natively unfolded protein. J Biol Chem, 277(36):33447-55.

Devos, D., Dokudovskaya, S., Alber, F., Williams, R., Chait, B. T., Sali, A., and Rout, M. P. (2004). Components of coated vesicles and nuclear pore complexes share a common molecular architecture. PLoS Biol, 2(12):e380.

Dilworth, D. J., Suprapto, A., Padovan, J. C., Chait, B. T., Wozniak, R. W., Rout, M. P., and Aitchison, J. D. (2001). Nup2p dynamically associates with the distal regions of the yeast nuclear pore complex. J Cell Biol, 153(7):1465-78.

Eisele, N. B., Frey, S., Piehler, J., Görlich, D., and Richter, R. P. (2010). Ultrathin nucleoporin phenylalanine-glycine repeat films and their interaction with nuclear transport receptors. EMBO Rep, 11(5):366-72.

Fabre, E., Boelens, W. C., Wimmer, C., Mattaj, I. W., and Hurt, E. C. (1994). Nup145p is required for nuclear export of mRNA and binds homopolymeric RNA in vitro via a novel conserved motif. Cell, 78(2):275-89.

Fabre, E. and Hurt, E. (1997). Yeast genetics to dissect the nuclear pore complex and nucleocytoplasmic trafficking. Annu Rev Genet, 31:277-313.

Fabre, E., Schlaich, N. L., and Hurt, E. C. (1995). Nucleocytoplasmic trafficking: what role for repeated motifs in nucleoporins? Cold Spring Harb Symp Quant Biol, 60:677-85.

Feldherr, C. M. (1962). The nuclear annuli as pathways for nucleocytoplasmic exchanges. J Cell Biol, 14(1):65.

Fernandez-Martinez, J., Phillips, J., Sekedat, M. D., Diaz-Avalos, R., Velazquez-Muriel, J., Franke, J. D., Williams, R., Stokes, D. L., Chait, B. T., Sali, A., and Rout, M. P. (2012). Structurefunction mapping of a heptameric module in the nuclear pore complex. J Cell Biol, 196(4):41934.

Feuerbach, F., Galy, V., Trelles-Sticken, E., Fromont-Racine, M., Jacquier, A., Gilson, E., OlivoMarin, J.-C., Scherthan, H., and Nehrbass, U. (2002). Nuclear architecture and spatial positioning help establish transcriptional states of telomeres in yeast. Nature Cell Biology, 4(3):214-21.

Field, M. C. and Dacks, J. B. (2009). First and last ancestors: reconstructing evolution of the endomembrane system with ESCRTs, vesicle coat proteins, and nuclear pore complexes. Current opinion in cell biology, 21(1):4-13.

Finlay, D. R. and Forbes, D. J. (1990). Reconstitution of biochemically altered nuclear pores: transport can be eliminated and restored. Cell, 60(1):17-29.

Fontoura, B. M., Blobel, G., and Matunis, M. J. (1999). A conserved biogenesis pathway for nucleoporins: proteolytic processing of a 186-kilodalton precursor generates Nup98 and the novel nucleoporin, Nup96. J Cell Biol, 144(6):1097-112. 
Frey, S. and Görlich, D. (2007). A saturated FG-repeat hydrogel can reproduce the permeability properties of nuclear pore complexes. Cell, 130(3):512-23.

Frey, S. and Görlich, D. (2009). FG/FxFG as well as GLFG repeats form a selective permeability barrier with self-healing properties. The EMBO Journal, 28(17):2554-67.

Frey, S., Richter, R. P., and Görlich, D. (2006). FG-rich repeats of nuclear pore proteins form a three-dimensional meshwork with hydrogel-like properties. Science (New York, NY), $314(5800): 815-7$.

Fried, H. and Kutay, U. (2003). Nucleocytoplasmic transport: taking an inventory. Cell Mol Life Sci, 60(8):1659-88.

Gall, J. G. (1967). Octagonal nuclear pores. J Cell Biol, 32(2):391-9.

Giaever, G., Chu, A. M., Ni, L., Connelly, C., Riles, L., Véronneau, S., Dow, S., Lucau-Danila, A., Anderson, K., André, B., Arkin, A. P., Astromoff, A., El-Bakkoury, M., Bangham, R., Benito, R., Brachat, S., Campanaro, S., Curtiss, M., Davis, K., Deutschbauer, A., Entian, K.-D., Flaherty, P., Foury, F., Garfinkel, D. J., Gerstein, M., Gotte, D., Güldener, U., Hegemann, J. H., Hempel, S., Herman, Z., Jaramillo, D. F., Kelly, D. E., Kelly, S. L., Kötter, P., LaBonte, D., Lamb, D. C., Lan, N., Liang, H., Liao, H., Liu, L., Luo, C., Lussier, M., Mao, R., Menard, P., Ooi, S. L., Revuelta, J. L., Roberts, C. J., Rose, M., Ross-Macdonald, P., Scherens, B., Schimmack, G., Shafer, B., Shoemaker, D. D., Sookhai-Mahadeo, S., Storms, R. K., Strathern, J. N., Valle, G., Voet, M., Volckaert, G., yun Wang, C., Ward, T. R., Wilhelmy, J., Winzeler, E. A., Yang, Y., Yen, G., Youngman, E., Yu, K., Bussey, H., Boeke, J. D., Snyder, M., Philippsen, P., Davis, R. W., and Johnston, M. (2002). Functional profiling of the Saccharomyces cerevisiae genome. Nature, 418(6896):387-91.

Gietz, R. D., Schiestl, R. H., Willems, A. R., and Woods, R. A. (1995). Studies on the transformation of intact yeast cells by the LiAc/SS-DNA/PEG procedure. Yeast, 11(4):355-60.

Goldstein, A. L., Snay, C. A., Heath, C. V., and Cole, C. N. (1996). Pleiotropic nuclear defects associated with a conditional allele of the novel nucleoporin Rat9p/Nup85p. Mol Biol Cell, $7(6): 917-34$.

Gomez-Ospina, N., Morgan, G., Giddings, T. H., Kosova, B., Hurt, E., and Winey, M. (2000). Yeast nuclear pore complex assembly defects determined by nuclear envelope reconstruction. $J$ Struct Biol, 132(1):1-5.

Görlich, D. and Kutay, U. (1999). Transport between the cell nucleus and the cytoplasm. Annu. Rev. Cell. Dev. Biol., 15:607-60.

Gorsch, L. C., Dockendorff, T. C., and Cole, C. N. (1995). A conditional allele of the novel repeatcontaining yeast nucleoporin RAT7/NUP159 causes both rapid cessation of mRNA export and reversible clustering of nuclear pore complexes. J Cell Biol, 129(4):939-55.

Grandi, P., Doye, V., and Hurt, E. C. (1993). Purification of NSP1 reveals complex formation with 'GLFG' nucleoporins and a novel nuclear pore protein NIC96. EMBO J, 12(8):3061-71. 
Grandi, P., Schlaich, N., Tekotte, H., and Hurt, E. C. (1995). Functional interaction of Nic96p with a core nucleoporin complex consisting of Nsp1p, Nup49p and a novel protein Nup57p. EMBO $J, 14(1): 76-87$.

Griesbeck, O., Baird, G. S., Campbell, R. E., Zacharias, D. A., and Tsien, R. Y. (2001). Reducing the environmental sensitivity of yellow fluorescent protein. Mechanism and applications. J Biol Chem, 276(31):29188-94.

Griffis, E. R., Xu, S., and Powers, M. A. (2003). Nup98 localizes to both nuclear and cytoplasmic sides of the nuclear pore and binds to two distinct nucleoporin subcomplexes. Mol Biol Cell, 14(2):600-10.

Grote, M., Kubitscheck, U., Reichelt, R., and Peters, R. (1995). Mapping of nucleoporins to the center of the nuclear pore complex by post-embedding immunogold electron microscopy. J Cell Sci, 108 (Pt 9):2963-72.

Gustin, K. E. and Sarnow, P. (2001). Effects of poliovirus infection on nucleo-cytoplasmic trafficking and nuclear pore complex composition. EMBO J, 20(1-2):240-9.

Gustin, K. E. and Sarnow, P. (2002). Inhibition of nuclear import and alteration of nuclear pore complex composition by rhinovirus. J Virol, 76(17):8787-96.

Hetzer, M. W., Walther, T. C., and Mattaj, I. W. (2005). Pushing the envelope: Structure, Function, and Dynamics of the Nuclear Periphery. Annu. Rev. Cell. Dev. Biol., 21(1):347-380.

Hinshaw, J. E., Carragher, B. O., and Milligan, R. A. (1992). Architecture and design of the nuclear pore complex. Cell, 69(7):1133-41.

Ho, A. K., Shen, T. X., Ryan, K. J., Kiseleva, E., Levy, M. A., Allen, T. D., and Wente, S. R. (2000). Assembly and preferential localization of Nup116p on the cytoplasmic face of the nuclear pore complex by interaction with Nup82p. Mol Cell Biol, 20(15):5736-48.

Hodel, A. E., Hodel, M. R., Griffis, E. R., Hennig, K. A., Ratner, G. A., Xu, S., and Powers, M. A. (2002). The three-dimensional structure of the autoproteolytic, nuclear pore-targeting domain of the human nucleoporin Nup98. Molecular Cell, 10(2):347-58.

Hurt, E. C. (1988). A novel nucleoskeletal-like protein located at the nuclear periphery is required for the life cycle of Saccharomyces cerevisiae. The EMBO Journal, 7(13):4323-34.

Hurwitz, M. E., de Castillia, C. S., and Blobel, G. (1998). Two yeast nuclear pore complex proteins involved in mRNA export form a cytoplasmically oriented subcomplex. Proc Natl Acad Sci USA, 95(19):11241-5.

Iovine, M. K., Watkins, J. L., and Wente, S. R. (1995). The GLFG repetitive region of the nucleoporin Nup116p interacts with Kap95p, an essential yeast nuclear import factor. J Cell Biol, 131(6 Pt 2):1699-713.

Iovine, M. K. and Wente, S. R. (1997). A nuclear export signal in Kap95p is required for both recycling the import factor and interaction with the nucleoporin GLFG repeat regions of Nup116p and Nup100p. J Cell Biol, 137(4):797-811. 
Isgro, T. A. and Schulten, K. (2005). Binding dynamics of isolated nucleoporin repeat regions to importin-beta. Structure, 13(12):1869-79.

Johnston, J. and Mortimer, R. (1959). Use of snail digestive juice in isolation of yeast spore tetrads. J Bacteriol, 78:292.

Jovanovic-Talisman, T., Tetenbaum-Novatt, J., McKenney, A. S., Zilman, A., Peters, R., Rout, M. P., and Chait, B. T. (2009). Artificial nanopores that mimic the transport selectivity of the nuclear pore complex. Nature, 457(7232):1023-7.

Kassir, Y. and Simchen, G. (1991). Monitoring meiosis and sporulation in Saccharomyces cerevisiae. Meth Enzymol, 194:94-110.

Kenna, M. A., Petranka, J. G., Reilly, J. L., and Davis, L. I. (1996). Yeast N1e3p/Nup170p is required for normal stoichiometry of FG nucleoporins within the nuclear pore complex. Mol Cell Biol, 16(5):2025-36.

Kiseleva, E., Allen, T. D., Rutherford, S., Bucci, M., Wente, S. R., and Goldberg, M. W. (2004). Yeast nuclear pore complexes have a cytoplasmic ring and internal filaments. J Struct Biol, $145(3): 272-88$.

Kiseleva, E., Goldberg, M. W., Allen, T. D., and Akey, C. W. (1998). Active nuclear pore complexes in Chironomus: visualization of transporter configurations related to mRNP export. J Cell Sci, 111 (Pt 2):223-36.

Kölling, R., Nguyen, T., Chen, E. Y., and Botstein, D. (1993). A new yeast gene with a myosin-like heptad repeat structure. Mol Gen Genet, 237(3):359-69.

Kosova, B., Panté, N., Rollenhagen, C., and Hurt, E. (1999). Nup192p is a conserved nucleoporin with a preferential location at the inner site of the nuclear membrane. $J$ Biol Chem, $274(32): 22646-51$.

Kowalczyk, S. W., Kapinos, L., Blosser, T. R., Magalhães, T., van Nies, P., Lim, R. Y. H., and Dekker, C. (2011). Single-molecule transport across an individual biomimetic nuclear pore complex. Nature nanotechnology, 6(7):433-8.

Kubitscheck, U., Grünwald, D., Hoekstra, A., Rohleder, D., Kues, T., Siebrasse, J. P., and Peters, R. (2005). Nuclear transport of single molecules: dwell times at the nuclear pore complex. $J$ Cell Biol, 168(2):233-43.

Laurell, E., Beck, K., Krupina, K., Theerthagiri, G., Bodenmiller, B., Horvath, P., Aebersold, R., Antonin, W., and Kutay, U. (2011). Phosphorylation of Nup98 by multiple kinases is crucial for NPC disassembly during mitotic entry. Cell, 144(4):539-50.

Lim, R. Y. H., Fahrenkrog, B., Koser, J., Schwarz-Herion, K., Deng, J., and Aebi, U. (2007). Nanomechanical Basis of Selective Gating by the Nuclear Pore Complex. Science, 318(5850):640643. 
Lim, R. Y. H., Huang, N.-P., Köser, J., Deng, J., Lau, K. H. A., Schwarz-Herion, K., Fahrenkrog, B., and Aebi, U. (2006). Flexible phenylalanine-glycine nucleoporins as entropic barriers to nucleocytoplasmic transport. Proc Natl Acad Sci USA, 103(25):9512-7.

Lusk, C. P., Makhnevych, T., Marelli, M., Aitchison, J. D., and Wozniak, R. W. (2002). Karyopherins in nuclear pore biogenesis: a role for Kap121p in the assembly of Nup53p into nuclear pore complexes. J Cell Biol, 159(2):267-78.

Lutzmann, M., Kunze, R., Buerer, A., Aebi, U., and Hurt, E. (2002). Modular self-assembly of a Y-shaped multiprotein complex from seven nucleoporins. EMBO J, 21(3):387-97.

Lutzmann, M., Kunze, R., Stangl, K., Stelter, P., Tóth, K. F., Böttcher, B., and Hurt, E. (2005). Reconstitution of Nup157 and Nup145N into the Nup84 complex. J Biol Chem, 280(18):1844251.

Madrid, A. S., Mancuso, J., Cande, W. Z., and Weis, K. (2006). The role of the integral membrane nucleoporins Ndc1p and Pom152p in nuclear pore complex assembly and function. J Cell Biol, 173(3):361-71.

Makio, T., Stanton, L. H., Lin, C.-C., Goldfarb, D. S., Weis, K., and Wozniak, R. W. (2009). The nucleoporins Nup170p and Nup157p are essential for nuclear pore complex assembly. J Cell Biol, 185(3):459-73.

Meinema, A. C., Laba, J. K., Hapsari, R. A., Otten, R., Mulder, F. A. A., Kralt, A., van den Bogaart, G., Lusk, C. P., Poolman, B., and Veenhoff, L. M. (2011). Long unfolded linkers facilitate membrane protein import through the nuclear pore complex. Science (New York, $N Y), 333(6038): 90-3$.

Miao, L. and Schulten, K. (2009). Transport-related structures and processes of the nuclear pore complex studied through molecular dynamics. Structure, 17(3):449-59.

Miao, M., Ryan, K. J., and Wente, S. R. (2006). The integral membrane protein Pom34p functionally links nucleoporin subcomplexes. Genetics, 172(3):1441-57.

Milles, S. and Lemke, E. A. (2011). Single molecule study of the intrinsically disordered FG-repeat nucleoporin 153. Biophys $J, 101(7): 1710-9$.

Mincer, J. S. and Simon, S. M. (2011). Simulations of nuclear pore transport yield mechanistic insights and quantitative predictions. Proc Natl Acad Sci USA, 108(31):E351-8.

Mohr, D., Frey, S., Fischer, T., Güttler, T., and Görlich, D. (2009). Characterisation of the passive permeability barrier of nuclear pore complexes. The EMBO Journal, 28(17):2541-53.

Moussavi-Baygi, R., Jamali, Y., Karimi, R., and Mofrad, M. R. K. (2011). Brownian dynamics simulation of nucleocytoplasmic transport: a coarse-grained model for the functional state of the nuclear pore complex. PLoS Comput Biol, 7(6):e1002049.

Mullis, K., Faloona, F., Scharf, S., Saiki, R., Horn, G., and Erlich, H. (1986). Specific enzymatic amplification of DNA in vitro: the polymerase chain reaction. Cold Spring Harb Symp Quant Biol, 51 Pt 1:263-73. 
Murphy, R., Watkins, J. L., and Wente, S. R. (1996). GLE2, a Saccharomyces cerevisiae homologue of the Schizosaccharomyces pombe export factor RAE1, is required for nuclear pore complex structure and function. Mol Biol Cell, 7(12):1921-37.

Nagy, V., Hsia, K.-C., Debler, E. W., Kampmann, M., Davenport, A. M., Blobel, G., and Hoelz, A. (2009). Structure of a trimeric nucleoporin complex reveals alternate oligomerization states. Proc Natl Acad Sci USA, 106(42):17693-8.

Nehrbass, U., Rout, M. P., Maguire, S., Blobel, G., and Wozniak, R. W. (1996). The yeast nucleoporin Nup188p interacts genetically and physically with the core structures of the nuclear pore complex. J Cell Biol, 133(6):1153-62.

Neumann, N., Lundin, D., and Poole, A. M. (2010). Comparative genomic evidence for a complete nuclear pore complex in the last eukaryotic common ancestor. PLoS ONE, 5(10):e13241.

Onischenko, E., Stanton, L. H., Madrid, A. S., Kieselbach, T., and Weis, K. (2009). Role of the Ndc1 interaction network in yeast nuclear pore complex assembly and maintenance. J Cell Biol, 185(3):475-91.

Onischenko, E. and Weis, K. (2011). Nuclear pore complex-a coat specifically tailored for the nuclear envelope. Current opinion in cell biology, 23(3):293-301.

Osmani, A. H., Davies, J., Liu, H.-L., Nile, A., and Osmani, S. A. (2006). Systematic deletion and mitotic localization of the nuclear pore complex proteins of Aspergillus nidulans. Mol Biol Cell, 17(12):4946-61.

Ovechkina, Y., Maddox, P., Oakley, C. E., Xiang, X., Osmani, S. A., Salmon, E. D., and Oakley, B. R. (2003). Spindle formation in Aspergillus is coupled to tubulin movement into the nucleus. Mol Biol Cell, 14(5):2192-200.

Paine, P. L. and Feldherr, C. M. (1972). Nucleocytoplasmic exchange of macromolecules. Exp Cell Res, 74(1):81-98.

Paine, P. L., Moore, L. C., and Horowitz, S. B. (1975). Nuclear envelope permeability. Nature, 254(5496):109-14.

Palmer, B. R. and Marinus, M. G. (1994). The dam and dcm strains of Escherichia coli-a review. Gene, 143(1):1-12.

Park, N., Katikaneni, P., Skern, T., and Gustin, K. E. (2008). Differential targeting of nuclear pore complex proteins in poliovirus-infected cells. J Virol, 82(4):1647-55.

Patel, S. S., Belmont, B. J., Sante, J. M., and Rexach, M. F. (2007). Natively unfolded nucleoporins gate protein diffusion across the nuclear pore complex. Cell, 129(1):83-96.

Peters, R. (2005). Translocation through the nuclear pore complex: selectivity and speed by reduction-of-dimensionality. Traffic, 6(5):421-7.

Peters, R. (2009). Translocation through the nuclear pore: Kaps pave the way. Bioessays, $31(4): 466-77$. 
Powers, M. A., Macaulay, C., Masiarz, F. R., and Forbes, D. J. (1995). Reconstituted nuclei depleted of a vertebrate GLFG nuclear pore protein, p97, import but are defective in nuclear growth and replication. J Cell Biol, 128(5):721-36.

Priore, V. D., Heath, C., Snay, C., MacMillan, A., Gorsch, L., Dagher, S., and Cole, C. (1997). A structure/function analysis of Rat7p/Nup159p, an essential nucleoporin of Saccharomyces cerevisiae. J Cell Sci, 110 (Pt 23):2987-99.

Pritchard, C. E., Fornerod, M., Kasper, L. H., and van Deursen, J. M. (1999). RAE1 is a shuttling mRNA export factor that binds to a GLEBS-like NUP98 motif at the nuclear pore complex through multiple domains. J Cell Biol, 145(2):237-54.

Radu, A., Moore, M. S., and Blobel, G. (1995). The peptide repeat domain of nucleoporin Nup98 functions as a docking site in transport across the nuclear pore complex. Cell, 81(2):215-22.

Ratner, G. A., Hodel, A. E., and Powers, M. A. (2007). Molecular Determinants of Binding between Gly-Leu-Phe-Gly Nucleoporins and the Nuclear Pore Complex. Journal of Biological Chemistry, 282(47):33968-33976.

Reichelt, R., Holzenburg, A., Buhle, E. L., Jarnik, M., Engel, A., and Aebi, U. (1990). Correlation between structure and mass distribution of the nuclear pore complex and of distinct pore complex components. J Cell Biol, 110(4):883-94.

Ribbeck, K. and Görlich, D. (2001). Kinetic analysis of translocation through nuclear pore complexes. EMBO J, 20(6):1320-30.

Ribbeck, K. and Görlich, D. (2002). The permeability barrier of nuclear pore complexes appears to operate via hydrophobic exclusion. EMBO J, 21(11):2664-71.

Rout, M. P., Aitchison, J. D., Magnasco, M. O., and Chait, B. T. (2003). Virtual gating and nuclear transport: the hole picture. Trends in Cell Biology, 13(12):622-8.

Rout, M. P., Aitchison, J. D., Suprapto, A., Hjertaas, K., Zhao, Y., and Chait, B. T. (2000). The yeast nuclear pore complex: composition, architecture, and transport mechanism. J Cell Biol, 148(4):635-51.

Rout, M. P. and Blobel, G. (1993). Isolation of the yeast nuclear pore complex. J Cell Biol, 123(4):771-83.

Rout, M. P. and Wente, S. R. (1994). Pores for thought: nuclear pore complex proteins. Trends in Cell Biology, 4(10):357-65.

Saavedra, C. A., Hammell, C. M., Heath, C. V., and Cole, C. N. (1997). Yeast heat shock mRNAs are exported through a distinct pathway defined by Rip1p. Genes Dev, 11(21):2845-56.

Sambrook, J. and Russell, D. W. (2001). Molecular Cloning: A Laboratory Manual. Cold Spring Harbor Laboratory Press, NY, (3).

Sauer, B. (1987). Functional expression of the cre-lox site-specific recombination system in the yeast Saccharomyces cerevisiae. Mol Cell Biol, 7(6):2087-96. 
Sauer, B. (1998). Inducible gene targeting in mice using the Cre/lox system. Methods, 14(4):381-92.

Schlaich, N. L., Häner, M., Lustig, A., Aebi, U., and Hurt, E. C. (1997). In vitro reconstitution of a heterotrimeric nucleoporin complex consisting of recombinant Nsp1p, Nup49p, and Nup57p. Mol Biol Cell, 8(1):33-46.

Schrader, N., Stelter, P., Flemming, D., Kunze, R., Hurt, E., and Vetter, I. R. (2008). Structural basis of the nic96 subcomplex organization in the nuclear pore channel. Molecular Cell, 29(1):4655 .

Sherman, F. (2002). Getting started with yeast. Meth Enzymol, 350:3-41.

Sikorski, R. S. and Hieter, P. (1989). A system of shuttle vectors and yeast host strains designed for efficient manipulation of DNA in Saccharomyces cerevisiae. Genetics, 122(1):19-27.

Singh, M. V. and Weil, P. A. (2002). A method for plasmid purification directly from yeast. Anal Biochem, 307(1):13-7.

Siniossoglou, S., Lutzmann, M., Santos-Rosa, H., Leonard, K., Mueller, S., Aebi, U., and Hurt, E. (2000). Structure and assembly of the Nup84p complex. J Cell Biol, 149(1):41-54.

Siniossoglou, S., Wimmer, C., Rieger, M., Doye, V., Tekotte, H., Weise, C., Emig, S., Segref, A., and Hurt, E. C. (1996). A novel complex of nucleoporins, which includes Sec13p and a Sec13p homolog, is essential for normal nuclear pores. Cell, 84(2):265-75.

Snow, C. M., Senior, A., and Gerace, L. (1987). Monoclonal antibodies identify a group of nuclear pore complex glycoproteins. J Cell Biol, 104(5):1143-56.

Souza, C. P. C. D., Osmani, A. H., Hashmi, S. B., and Osmani, S. A. (2004). Partial nuclear pore complex disassembly during closed mitosis in Aspergillus nidulans. Curr Biol, 14(22):1973-84.

Stelter, P., Kunze, R., Flemming, D., Höpfner, D., Diepholz, M., Philippsen, P., Böttcher, B., and Hurt, E. (2007). Molecular basis for the functional interaction of dynein light chain with the nuclear-pore complex. Nat Cell Biol, 9(7):788-96.

Stoffler, D., Feja, B., Fahrenkrog, B., Walz, J., Typke, D., and Aebi, U. (2003). Cryo-electron tomography provides novel insights into nuclear pore architecture: implications for nucleocytoplasmic transport. Journal of Molecular Biology, 328(1):119-30.

Strawn, L. A., Shen, T., Shulga, N., Goldfarb, D. S., and Wente, S. R. (2004). Minimal nuclear pore complexes define FG repeat domains essential for transport. Nat Cell Biol, 6(3):197-206.

Stutz, F., Kantor, J., Zhang, D., McCarthy, T., Neville, M., and Rosbash, M. (1997). The yeast nucleoporin rip1p contributes to multiple export pathways with no essential role for its FG-repeat region. Genes Dev, 11(21):2857-68.

Stutz, F., Neville, M., and Rosbash, M. (1995). Identification of a novel nuclear pore-associated protein as a functional target of the HIV-1 Rev protein in yeast. Cell, 82(3):495-506. 
Suelmann, R., Sievers, N., and Fischer, R. (1997). Nuclear traffic in fungal hyphae: in vivo study of nuclear migration and positioning in Aspergillus nidulans. Mol Microbiol, 25(4):757-69.

Teixeira, M. T., Siniossoglou, S., Podtelejnikov, S., Bénichou, J. C., Mann, M., Dujon, B., Hurt, E., and Fabre, E. (1997). Two functionally distinct domains generated by in vivo cleavage of Nup145p: a novel biogenesis pathway for nucleoporins. EMBO J, 16(16):5086-97.

Terry, L. J. and Wente, S. R. (2007). Nuclear mRNA export requires specific FG nucleoporins for translocation through the nuclear pore complex. J Cell Biol, 178(7):1121-32.

Terry, L. J. and Wente, S. R. (2009). Flexible gates: dynamic topologies and functions for FG nucleoporins in nucleocytoplasmic transport. Eukaryotic Cell, 8(12):1814-27.

Thomas, J. H. and Botstein, D. (1986). A gene required for the separation of chromosomes on the spindle apparatus in yeast. Cell, 44(1):65-76.

Wälde, S. and Kehlenbach, R. H. (2010). The Part and the Whole: functions of nucleoporins in nucleocytoplasmic transport. Trends in Cell Biology, 20(8):461-9.

Weirich, C. S., Erzberger, J. P., Berger, J. M., and Weis, K. (2004). The N-terminal domain of Nup159 forms a beta-propeller that functions in mRNA export by tethering the helicase Dbp5 to the nuclear pore. Molecular Cell, 16(5):749-60.

Wente, S. R. and Blobel, G. (1993). A temperature-sensitive NUP116 null mutant forms a nuclear envelope seal over the yeast nuclear pore complex thereby blocking nucleocytoplasmic traffic. $J$ Cell Biol, 123(2):275-84.

Wente, S. R. and Blobel, G. (1994). NUP145 encodes a novel yeast glycine-leucine-phenylalanineglycine (GLFG) nucleoporin required for nuclear envelope structure. J Cell Biol, 125(5):955-69.

Wente, S. R., Rout, M. P., and Blobel, G. (1992). A new family of yeast nuclear pore complex proteins. J Cell Biol, 119(4):705-23.

Whittle, J. R. R. and Schwartz, T. U. (2009). Architectural nucleoporins Nup157/170 and Nup133 are structurally related and descend from a second ancestral element. Journal of Biological Chemistry, 284(41):28442-52.

Wimmer, C., Doye, V., Grandi, P., Nehrbass, U., and Hurt, E. C. (1992). A new subclass of nucleoporins that functionally interact with nuclear pore protein NSP1. EMBO J, 11(13):505161.

Winey, M., Hoyt, M. A., Chan, C., Goetsch, L., Botstein, D., and Byers, B. (1993). NDC1: a nuclear periphery component required for yeast spindle pole body duplication. J Cell Biol, $122(4): 743-51$.

Wischnitzer, S. (1958). An electron microscope study of the nuclear envelope of amphibian oocytes. J Ultrastruct Res, 1(3):201-22.

Wozniak, R. W., Blobel, G., and Rout, M. P. (1994). POM152 is an integral protein of the pore membrane domain of the yeast nuclear envelope. J Cell Biol, 125(1):31-42. 
Yamada, J., Phillips, J. L., Patel, S., Goldfien, G., Calestagne-Morelli, A., Huang, H., Reza, R., Acheson, J., Krishnan, V. V., Newsam, S., Gopinathan, A., Lau, E. Y., Colvin, M. E., Uversky, V. N., and Rexach, M. F. (2010). A bimodal distribution of two distinct categories of intrinsically disordered structures with separate functions in FG nucleoporins. Mol Cell Proteomics, 9(10):2205-24.

Yang, Q., Rout, M., and Akey, C. (1998). Three-dimensional architecture of the isolated yeast nuclear pore complex: functional and evolutionary implications. Molecular Cell, 1(2):223-234.

Yang, W. (2011). 'Natively unfolded' nucleoporins in nucleocytoplasmic transport: Clustered or evenly distributed? Nucleus (Austin, Tex), 2(1):10-16.

Yang, W. and Musser, S. M. (2006). Nuclear import time and transport efficiency depend on importin beta concentration. J Cell Biol, 174(7):951-61.

Yoshida, K., Seo, H.-S., Debler, E. W., Blobel, G., and Hoelz, A. (2011). Structural and functional analysis of an essential nucleoporin heterotrimer on the cytoplasmic face of the nuclear pore complex. Proc Natl Acad Sci USA, 108(40):16571-6.

Zabel, U., Doye, V., Tekotte, H., Wepf, R., Grandi, P., and Hurt, E. C. (1996). Nic96p is required for nuclear pore formation and functionally interacts with a novel nucleoporin, Nup188p. J Cell Biol, 133(6):1141-52.

Zeitler, B. and Weis, K. (2004). The FG-repeat asymmetry of the nuclear pore complex is dispensable for bulk nucleocytoplasmic transport in vivo. J Cell Biol, 167(4):583-90. 


\section{Abbreviations}

\begin{tabular}{|c|c|}
\hline aa & Amino acid \\
\hline ATP & Adenosine triphosphate \\
\hline $\mathrm{bp}$ & Base pairs \\
\hline $\mathrm{BSA}$ & Bovine serum albumin \\
\hline dATP & Deoxyadenosine triphosphate \\
\hline $\mathrm{dCTP}$ & Deoxycytosine triphosphate \\
\hline dGTP & Deoxyguanosine triphosphate \\
\hline DID & Dynein light chain interacting domain \\
\hline DMSO & Dimethylsulfoxide \\
\hline DNA & Deoxyribonucleic acid \\
\hline dpi & Dots per inch \\
\hline DTT & Dithiothreitol \\
\hline dTTP & Deoxythymidine triphosphate \\
\hline EDTA & Ethylenediaminetetraacetic acid \\
\hline FG & Phenylalanine-glycine \\
\hline FOA & 5'-Fluoroorotic Acid \\
\hline GLEBS & Gle2p binding site \\
\hline hr & Hour(s) \\
\hline $\min$ & Minute(s) \\
\hline mRNA & Messenger ribonucleic acid \\
\hline $\mathrm{N}-$ & Amino- \\
\hline $\mathrm{NE}$ & Nuclear envelope \\
\hline $\mathrm{NPC}$ & Nuclear pore complex \\
\hline NRM & Nucleoporin RNA-binding motif \\
\hline NTR & Nuclear transport receptor \\
\hline nup & Nucleoporin \\
\hline $\mathrm{OD}$ & Optical density at $600 \mathrm{~nm}$ \\
\hline PCR & Polymerase chain reaction \\
\hline rpm & Rounds per minute \\
\hline $\mathrm{RT}$ & Room temperature \\
\hline SDS & Sodium dodecyl sulphate \\
\hline sec & Second(s) \\
\hline Tris & Tris(hydroxymethyl)aminomethane \\
\hline $\mathrm{v} / \mathrm{v}$ & Volume/volume \\
\hline $\mathrm{w} / \mathrm{v}$ & Weight/volume \\
\hline
\end{tabular}

